\title{
WestVirginiaUniversity
}

THE RESEARCH REPOSITORY @ WVU

Graduate Theses, Dissertations, and Problem Reports

2010

\section{Essays on risk management applications to Appalachian grass -fed beef}

\author{
Maitreyi Mandal \\ West Virginia University
}

Follow this and additional works at: https://researchrepository.wvu.edu/etd

\section{Recommended Citation}

Mandal, Maitreyi, "Essays on risk management applications to Appalachian grass -fed beef" (2010). Graduate Theses, Dissertations, and Problem Reports. 4629.

https://researchrepository.wvu.edu/etd/4629

This Dissertation is protected by copyright and/or related rights. It has been brought to you by the The Research Repository @ WVU with permission from the rights-holder(s). You are free to use this Dissertation in any way that is permitted by the copyright and related rights legislation that applies to your use. For other uses you must obtain permission from the rights-holder(s) directly, unless additional rights are indicated by a Creative Commons license in the record and/ or on the work itself. This Dissertation has been accepted for inclusion in WVU Graduate Theses, Dissertations, and Problem Reports collection by an authorized administrator of The Research Repository @ WVU.

For more information, please contact researchrepository@mail.wvu.edu. 


\title{
ESSAYS ON RISK MANAGEMENT APPLICATIONS TO APPALACHIAN GRASS-FED BEEF
}

\author{
By \\ MAITREYI MANDAL
}

\author{
Dissertation submitted to the \\ Davis College of Agriculture, Natural Resources and Design \\ At West Virginia University \\ In partial fulfillment of the requirements for the degree of \\ Doctor of Philosophy \\ In \\ Natural Resource Economics
}

Gerard E. D'Souza, Ph.D., Chair

Jason R. Evans, Ph.D., Co-Chair

Cheryl Brown, Ph.D.

Peter Schaeffer, Ph.D.

Edward B. Rayburn, Ph.D.

\section{Agricultural and Resource Economics Program \\ Division of Resource Management \\ Morgantown, WV \\ 2010}

Keywords: risk, stochastic dominance, grass-fed beef, derivatives, weather. 


\begin{abstract}
ESSAYS ON RISK MANAGEMENT APPLICATIONS TO APPALACHIAN GRASS-FED BEEF
\end{abstract}

By

Maitreyi Mandal

The study area of this dissertation is the Appalachian state of West Virginia, where pasture-based livestock has traditionally dominated the state's agricultural production sector. Enhanced production risk has been identified as one of the key risk factors in grass fed beef production. This study aims to look into various risk management options that are available to grass-fed beef producers. One such management option is the various livestock insurance and rangeland, forage and grass land protection policies recently introduced and marketed by the Risk Management Agency (RMA) of USDA. The Livestock Risk Protection Plan (LRP) has been available to West Virginia cattle producers since summer 2006. This plan is uniquely aimed towards price protection. At the same time, two types of rangeland forage and pasture land protection policies have also been introduced by RMA, namely rainfall index and vegetation index based protection policies. These policies may help mitigate production related risk. A brief internet-based survey was conducted in early summer 2009 of various grass-fed beef producers who market their product electronically in neighboring states including West Virginia, Virginia, Pennsylvania, Kentucky, and Maryland to understand their perception of risk related to grass-fed production. It was found that drought or lack of rainfall is a major concern in their production related activities, an issue that further justified the need for this study. The latter consists of three distinct, but interrelated, essays, all oriented toward a better understanding of 
the causes, effects, and management of production risk in pasture-beef production settings. Stochastic dominance was the main tool used in the first and second essays to analyze various risky alternatives.

In the first essay, stochastic dominance analysis was used to understand LRP and price risk as may be faced by a grass-fed producer in West Virginia. It was found that in the current scenario, a high basis exists between the AMS 5 market selling price (on which the indemnity payouts are based) and the local cattle auction markets of Virginia and Pennsylvania. Due to this high basis, this LRP may not be suitable to these producers as offered currently.

The second essay deals with production risk and the rainfall index based protection policy of RMA. It was found that though this policy indeed helps to mitigate a certain part of production risk, managers of pastureland should have a thorough understanding of the nature of indemnity payouts. However, this insurance is not yet available in West Virginia. Hence, the study was conducted with Pennsylvania hay trial plots, using data provided by the WVU Agronomy Department.

As discussed earlier, in our e-mail based survey, it was found that drought or lack of rainfall is the major concern for most producers in Appalachia. Accordingly, in the third essay, a unique rainfall/precipitation based option contract was structured to assist businesses better manage rainfall/precipitation related risks. This unique precipitation based call/put option can be exploited by relevant businesses who suffer loss from either too little or too much precipitation. It was further found that there are indeed interested players in the capital market (e.g. Goldman Sachs commodity risk management; Phibro commodities trading firm; etc.) who are willing to underwrite the risk and provide such (rainfall call/put) risk management options to a broad range of businesses including grass-fed producers. This rainfall call/put, when made available through 
the capital market (underwriter) either as over the counter (OTC) or exchange (CME) traded, would provide yet another weather related risk management option to businesses in West Virginia affected by too much or too little rain fall/precipitation. 


\section{ACKNOWLEDGMENTS}

This dissertation has been made possible through funding from USDA-ARS for the project titled, "Economic Pasture-Based Beef Systems" for Appalachia. This project is a consortium among three academic institutes namely Virginia Tech, West Virginia University and Clemson University, with USDA-ARS as a collaborator. This funding is very much appreciated.

I am sincerely thankful to my academic advisor and supervisor of my academic program, Dr. Gerard D'Souza. I am also thankful to Dr.Oh and Dr. Brown to agree to work with me, as well as to Dr. Schaeffer for graciously agreeing to join the committee due to the departure from WVU of one of my committee members. Special thanks go to Dr. Jason Evans, Co-chair of my graduate committee. This dissertation would not take shape without his invaluable guidance, insight and helpful comments. Similarly, I am indebted to Dr. Edward Rayburn for providing me with all the relevant NOAA weather and hay yield data pertaining to Pennsylvania's agronomy trial plots. Sincere thanks goes to Dr. Oh for her insightful direction to rainfall modeling using Markovian chain, something I aim to learn more about in the near future.

Sincere thanks also go to Dr. Chuck Moss, Professor, Food and Resource Economics Dept., University of Florida. It was his class in Risk Analysis (AEB 6182) that provided the idea of using stochastic dominance in this analysis. Similarly, special mention needs to be made of Haas School of Business, University of California at Berkeley (UCB). After attending a presentation by Weather Bill (www.weatherbill.com), a firm which provides weather related insurance coverage, at The Masters of Financial Engineering (MFE) lab, Haas/UCB on $2^{\text {nd }}$ October, 2007, provided the inspiring idea to work on weather derivatives. Computational finance/Financial Engineering is the area I am highly interested to study further. Also, I am indebted to the following companies for providing derivatives-related data used in this analysis: Goldman Sachs

(Commodities Desk, NYC/London); Travelers (Hartford, CT); Phibro (Westport, CT); 
PIMCO/FAMCO (SanFransisco/St.Louis); and Moody's/S\&P (NYC). I am grateful to Ryan Benninger who helped me with proof reading an earlier draft. I am also thankful to Lisa Lewis, to help me out with timely submission of all etd related documents. Above all, I am grateful to my family and friends for constant encouragement and inspiration. Finally this dissertation is dedicated to my father who is the sole reason to complete my $\mathrm{PhD}$. 


\section{Table of Contents}

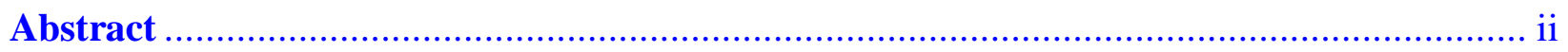

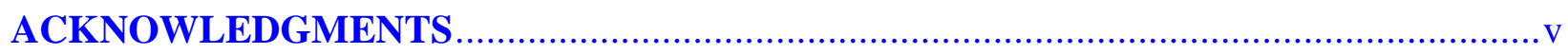

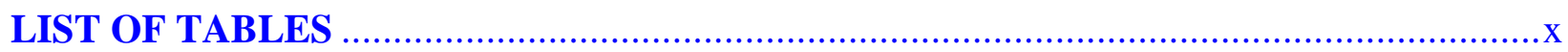

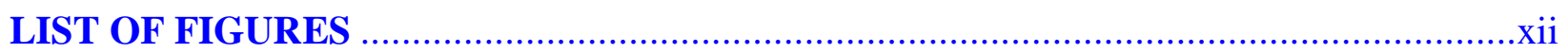

Relationship Among the Three Essays ..................................................................... xiii

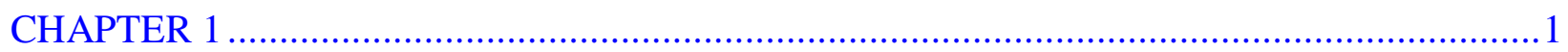

PRICE RISK AND LIVESTOCK PROTECTION PLAN (LRP) FROM USDA/RMA: A STOCHASTIC DOMINANCE ANALYSIS .............................................................

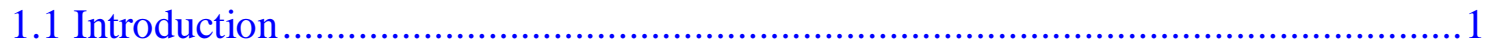

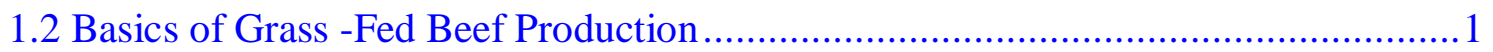

1.3 Livestock Protection Policies from USDA/RMA ……......................................... 3

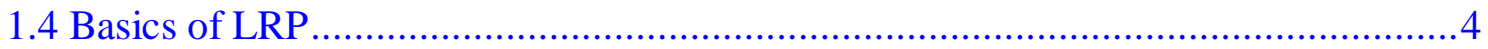

1.5. LRP Versus Cattle Put Options ....................................................................

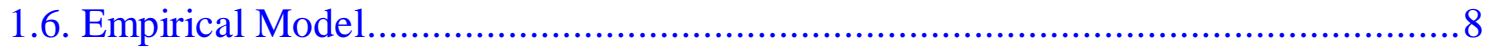

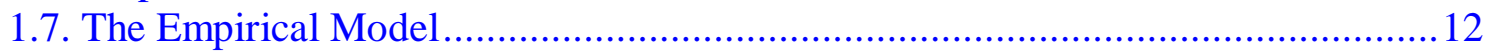

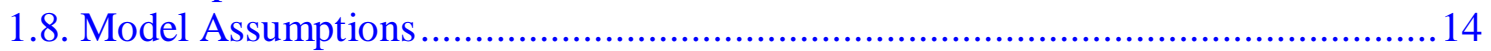

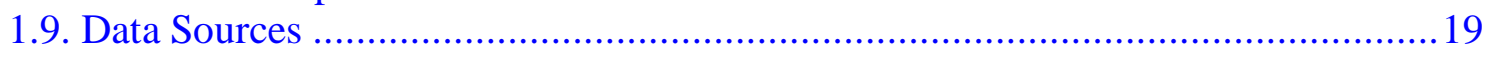

1.10. Basis in Local Cattle Market......................................................................19

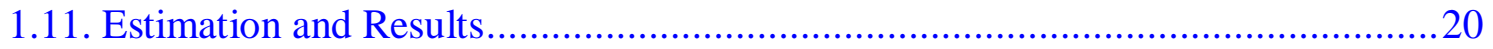

1.12. Black's (1976) Model to Price the LRP Premium ...............................................33

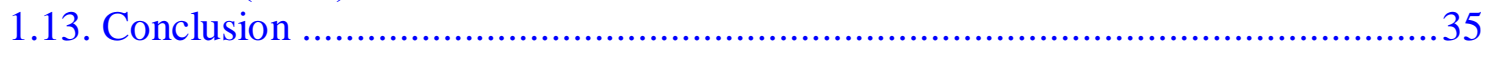

PRODUCTION RISK AND RAINFALL INSURANCE FROM USDA/RMA: A

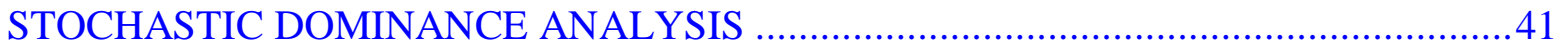

2. 1. Introduction .................................................................................................

2.2. Insurance for Pasture/Rangeland Management: Rainfall/Vegetation Index

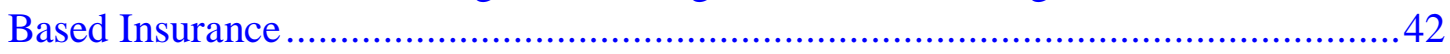

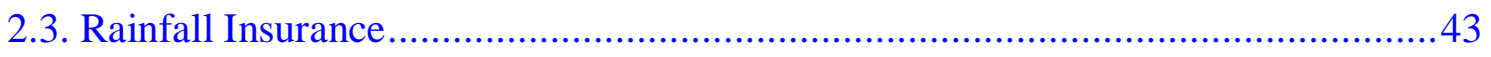

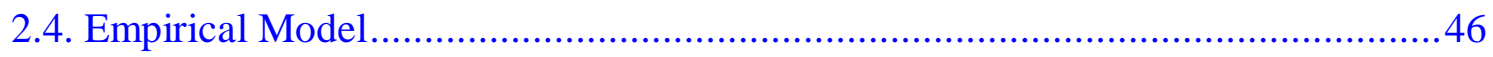

2.5. Model Assumptions for Stochastic Dominance Analysis of Essay II:....................48

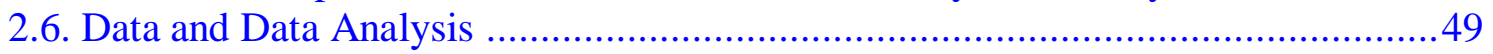

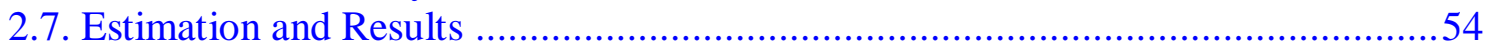

2.8. Climate Effects and Rainfall Index Insurance Purchase Decision ..........................57

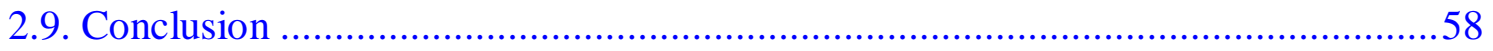

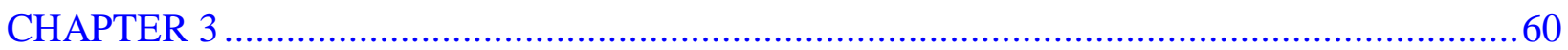




\section{STRUCTURING/MODELLING A PRECIPITATION OPTION (CALL/PuT) FOR WEST}

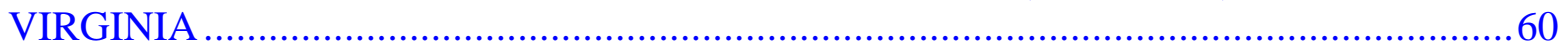

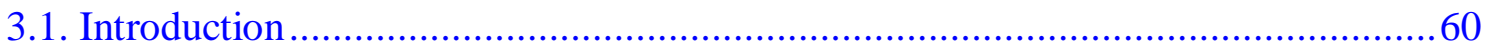

3.2. How Weather Derivatives Work ......................................................................62

3.3. Derivatives, Insurance and Reinsurance: A Review of Risk Management Tools ...62

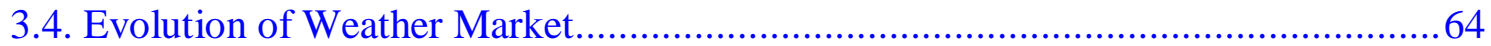

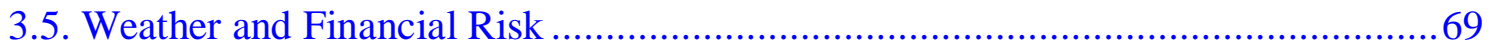

3.6. Different elements of a Weather Contract/Derivative ........................................... 70

3.7. Pay-off Diagram for Options ........................................................................ 71

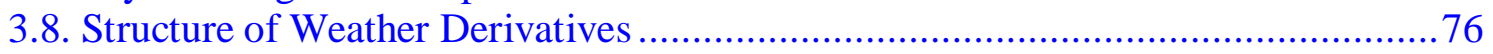

3.9. Other Possible Weather Derivative Structures (Combinations)................................79

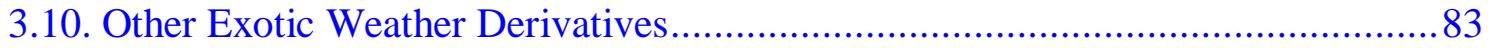

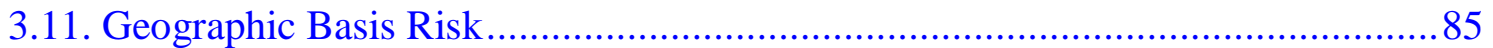

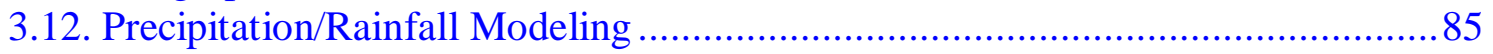

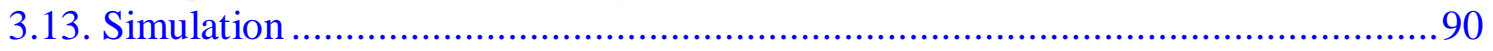

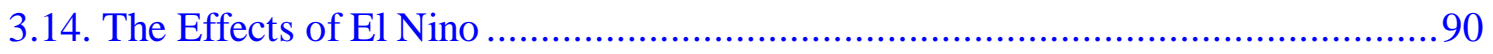

3.15. Rainfall/Precipitation Data, Estimation and Results ..........................................91

3.16. Derivative (Option) Pricing: Classical Methods .................................................. 96

3.17. Weather Derivative Valuation: A Review of Literature and Methods ................. 101

3.18. Previous Applications of Precipitation Contracts/Derivatives............................. 105

3.19. A Pricing Exercise by 'Burn' and Monte Carlo Simulation for the Current

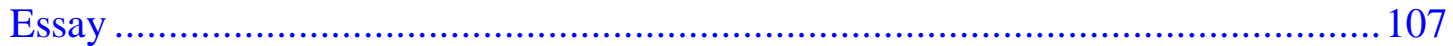

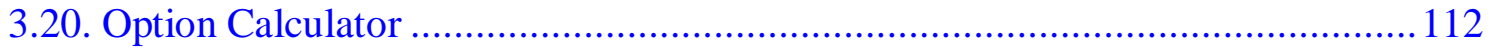

3.21. Possible Applications of the Structured Contract............................................... 112

3.22. Limitation of the Study and Suggested Derivative Strategies for Grass Fed

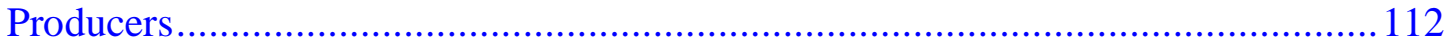

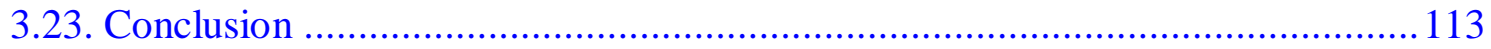

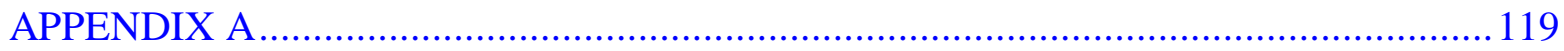

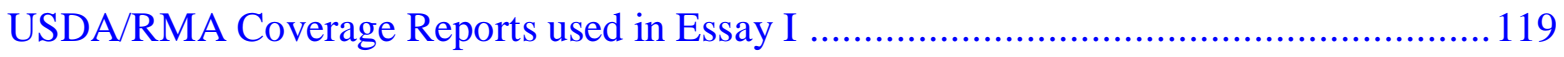

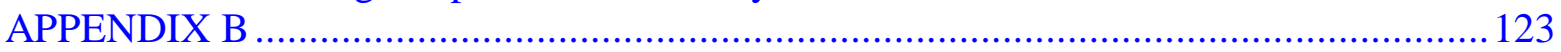

Decision Support Tool for Pasture, Rangeland and Forage ............................................ 123

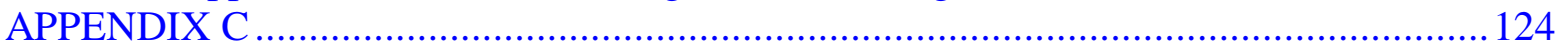

Basis Risk in Local Cattle Market vs. AMS 5 Area Market ............................................124

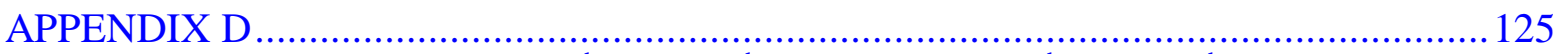

Actual LRP end value (\$) from $15^{\text {th }}$ Sep.- $15^{\text {th }}$ Oct., 2005 to $15^{\text {th }}$ Sep. $-15^{\text {th }}$ Oct., 2009 ........ 125

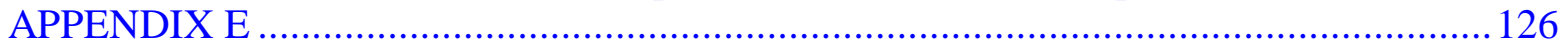

List of Weather Stations and Weather Futures Contracts ............................................... 126

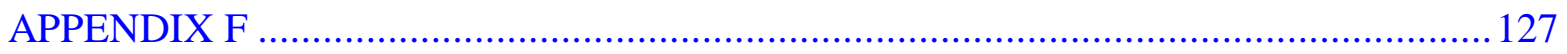

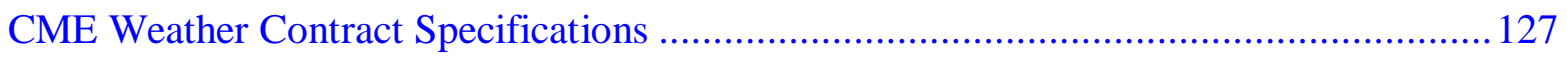

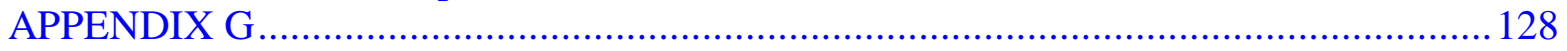

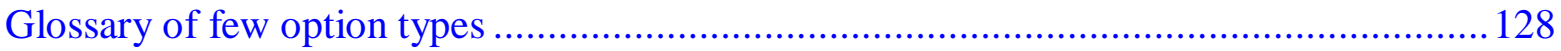

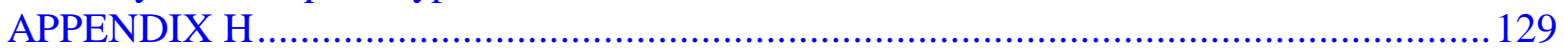

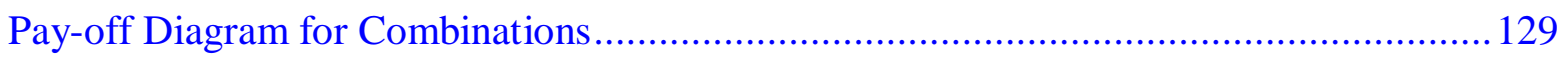

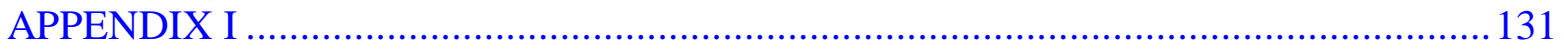

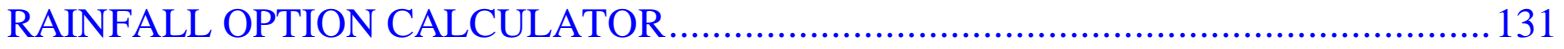

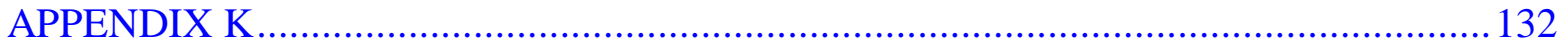


Grass-Fed Survey Questionnaire 


\section{LIST OF TABLES}

Table

Title

Page

1-1. Adjustments in CME Feeder Cattle Index for Cattle Size and 7

Type

1-2. Model Assumptions for Essay I 14

1-3. Various LRP Insurance choices with 10/15/2008 as the end 17

date

1-4. Various Return Scenarios 19

1-5. Data Sources for essay I 20

1-6. Descriptive Statistics of Net Return Distributions of 17

different insurances choices with Actual End Value as of 09/15/2008

1-7. Descriptive Statistics of Net Distribution Returns (End 25

Value: \$84.11)

1-8. Descriptive Statistics of Net Return Distributions of 17

different insurances choices

1-9. Risk Ranking of Case B with Actual End Value of \$84.11 32

1-10. Risk Ranking of Case B with Actual End Value of \$89.79 33

2-1. Model Assumptions for Essay II 49

2-2. Data Sources for Essay II $\quad 50$

2-3. Different Rainfall Insurance Choices available 51

2-4. Net Indemnity Received after Paying Premiums for GRP 51

Rainfall Insurance for Pastures for Interval III of Grid 24500(Grid I)

2-5. Net Indemnity Received after Paying Premiums for GRP 52

Rainfall Insurance for Pastures for Interval IV of Grid 24500 (Grid I)

2-6. Net Indemnity Received after Paying Premiums for GRP 52

Rainfall Insurance for Pastures for Interval III of Grid 24800 (Grid II)

2-7. Net Indemnity Received after Paying Premiums for GRP 53

Rainfall Insurance for Pastures for Interval IV of Grid 24800

(Grid II)

2-8. Descriptive Statistics of Net Return Distribution Grid $24500 \quad 53$

2-9. Descriptive Statistics of Net Return Distribution Grid $24800 \quad 54$

2-10. FSD Risk Ranking Grid $24500 \quad 55$

2-11. FSD Risk Rankings for Grid $24800 \quad 58$

3-1. How weather derivatives work 63

3-2. Links between Weather and Financial Risk $\quad 70$

3-3. Gamma Distribution Parameter Estimates Based on Daily 96

Precipitation of four WV Weather Stations

3-4. Exponential Distribution Parameter Estimates Based on 96

Daily Precipitation of four WV Weather Stations

3-5. Call Value at Expiry in \$ (Strike/Exercise rate is in rain 109 
inches)

3-6. Put Value at Expiry in \$ (Strike/Exercise rate is in precipitation inches) 


\section{LIST OF FIGURES}

Figure

$1-1$.

$1-2$.

$1-3$.

$1-4$.

$2-1$.

$2-2$.

$2-3$.

$2-4$.

2-5.

3-1

3-2. WTI Crude Oil Futures traded at NYMEX and ICE

3-3. Derivative Transaction Volume.

3-4. $\quad$ Pay-off diagram of long call for option buyer/holder

3-5. Pay-off diagram of long put for option holder/buyer

3-6. Pay-off diagram for short call for option seller

3-7. $\quad$ Pay-off diagram of short put for option seller

3-8. Pay-off diagram of HDD put

3-9. Precipitation Trend of Monongalia/Hartfield over 1971-2000

3-10. Annual Precipitation Trend between Monongalia/Hartfield and 93 Martinsburg/Berkeley (1971-2000)

3-11. Monthly Precipitation Trend-Monongalia/Hartfield vs. Martinsburg (1971- 93 2000)

3-12. Annual Cumulative Precipitation-Monongalia/Hartfield

3-13. Transition Probability for Monongalia $\quad 95$

3-14. Conditional Precipitation of Monongalia across days of year 95

3-15. Transition probability for Monroe $\quad 96$

3-16. Conditional Precipitation of Monroe across days of year 96

3-17. Geographic Basis Risk in Call Pricing (Burn Pricing) 108

3-18. Geographic Basis Risk in Call Pricing (Monte Carlo Pricing) 109

3-19. Geographic Basis Risk in Put Pricing (Burn Pricing) 112

3-20. Geographic Basis Risk in Put Pricing (Burn Pricing) 112

3-21. Flowchart of Option Calculator 113

3-22. Framework for Hedging Effectivess of Rainfall Option 114 


\section{RELATIONSHIP AMONG THE THREE ESSAYS}

Risk management is defined (by the ISO 31000 , for example) as the effect of uncertainty on objectives (whether positive or negative). Risk management can therefore be considered as the identification, assessment, and prioritization of risks followed by the coordinated and economical application of resources to minimize, monitor, and control the probability and/or impact of unfortunate events or to maximize the realization of opportunities. In this particular dissertation, a few aspects of risk, as perceived by grass- fed beef producers of Appalachia, have been identified, and the appropriateness of corresponding risk mitigation/reduction strategies through either purchase of government-offered insurance policies or unique market based instruments such as weather derivatives, have been evaluated. Grass-fed producers often have the dual role of both cattle-raiser and pasture/hay producer. In the first role, like any other producer, they face market/price risk. So, in the first essay, the role of the government-offered livestock risk plan which is a unique policy aimed towards protecting cattle producers from market risk, has been analyzed. The second essay analyzes the second role of these producers, which is as a hay producer. Finally, the third essay, examines the structuring of a unique financial product, i.e. a rainfall call/put/straddle based on local (West Virginia) weather station data. Weather-based contracts have been recently introduced in the Chicago Mercantile Exchange (CME) and have been growing in popularity, with rainfall contracts (obviously important to agriculturally-based settings) likely to be introduced in the not-too-distant future. In anticipation of this development, a hedging effectiveness strategy based on a structured rainfall contract is evaluated. It was very difficult to isolate the actual amount of revenue loss of these producers affected by too much or too little rainfall. Nonetheless, looking at rainfall-based 
derivative pricing is a first step towards structuring a weather based contract which is becoming more and more important in agricultural settings. 


\section{CHAPTER 1}

\section{PRICE RISK AND LIVESTOCK PROTECTION PLAN (LRP) FROM USDA/RMA: A STOCHASTIC DOMINANCE ANALYSIS}

If they give you ruled paper, write the other way

- Juan Ramon Jimenez

\subsection{Introduction}

Two types of livestock protection plans have been available to West Virginia cattle producers since summer of 2006. Due to the recent volatility in the cattle market, interest in price risk protection has increased. Traditionally, livestock producers have used either forward contracts or futures/options trading to manage this price risk. Livestock Risk Protection Plan (LRP) insurance provides another option that producers can now use to manage price risk. This essay is organized as follows: first, a brief description of both the LRP and Livestock Gross Margin (LGM) plans from the United States Department of Agriculture/Risk Management Agency (USDA/RMA) will be explained as supporting background perspective. Then an empirical model will be developed to determine the applicability of LRP for price risk management of grass-fed beef producers in West Virginia. Finally, we will examine the resulting industry and policy implications.

\subsection{Basics of Grass -Fed Beef Production}

In contrast to cattle produced via the conventional grain-intensive production system common here in the United Sates, grass-fed cattle are kept on natural pasture land for an extended period of time after the weaning period and spend no time in feedlots. Since forage-only diets typically contain less digestible energy and protein relative to grain diets, significantly longer periods are 
required to produce finished cattle utilizing grass-based systems. Animal performance and, consequently, finish weights of grass-fed cattle are contingent upon the quality and quantity of the forage available. It should be noted that forage availability is directly subject to the vagaries of both weather and other external variables. Spiselman (2006) states that grass-fed cattle generally do not reach a slaughter weight of $750 \mathrm{lbs}$. and higher until they are 24 to 30 months of age. On the contrary, it is known that grain-finished cattle are generally slaughtered at 14 to 18 months of age weighing 1200 to 1250 lbs., with particularly heavy slaughter activity in September and October of each year.

As per USDA guidelines, cattle marketed as "grass-fed" must meet the following guidelines:

"Grass and forage shall be the feed source consumed for the lifetime of the ruminant animal, with the exception of milk consumed prior to weaning. The diet shall be derived solely from forage consisting of grass (annual and perennial), forbs (e.g., legumes, Brassica), browse, or cereal grain crops in the vegetative (pre-grain) state. Animals cannot be fed grain or grain byproducts and must have continuous access to pasture during the growing season. Hay, haylage, baleage, silage, crop residue without grain, and other roughage sources may also be included as acceptable feed sources. Routine mineral and vitamin supplementation may also be included in the feeding regimen. If incidental supplementation occurs due to inadvertent exposure to non-forage feedstuffs or to ensure the animal's well being at all times during adverse environmental or physical conditions, the producer must fully document (e.g., receipts, ingredients, and tear tags) the supplementation that occurs including the amount, the frequency, and the supplements provided." (ams.usda.gov) 
Grass-fed production may have distinct advantages over conventional grain-based production in terms of market risk, given increased demand for corn from ethanol producers and uncertainty in feed prices. Further, live cattle market prices appear to be much more volatile than retail beef prices (Evans et al., 2007), indicating that producers who avoid marketing live cattle and instead sell harvested beef may experience less year-to-year revenue variability. Despite the apparent drawbacks of grass-finishing, namely lengthy growing periods and enhanced production risk, the resulting beef products have been shown to embody numerous relative benefits for human health, animal welfare, and the environment (Evans et al., 2007).

\subsection{Livestock Protection Policies from USDA/RMA}

Currently there are two types of insurance programs available.

\subsubsection{Livestock Gross Margin (LGM)}

The LGM for Cattle Insurance Policy provides protection against the loss of gross margin, which is the market value of livestock minus feeder cattle and feed costs associated with cattle. The indemnity at the end of the 11-month insurance period is the difference, if positive, between the gross margin guarantee and the actual gross margin. The LGM for Cattle Insurance Policy uses adjusted futures prices to determine the expected gross margin and the actual gross margin. Adjustments to futures prices are evaluated using both state- and month-specific basis levels. The price the producer receives at the local market is irrelevant in this price calculation (rma.usda.gov/livestock/). In this essay, we are not analyzing LGM, as typically a grass fed producer raises cattle in pasture and does not feed any grain based diets. So the corn future prices which are used to determine the expected gross margin and the actual gross margin are not applicable in our analysis. 


\subsubsection{Livestock Risk Protection (LRP)}

Under LRP, both feeder and fed cattle are insured against declining market prices. Cattle producers may select from a variety of coverage levels and insurance periods to correspond with the time their feeder steers and fed cattle would normally be marketed. An LRP insurance policy for both the feeder and fed cattle may be purchased throughout the year from approved livestock insurance agents. Premium rates, coverage prices, and actual ending values are posted online daily as found on the USDA/RMA website.

\subsection{Basics of LRP}

Since this essay deals with LRP, it is pertinent to gain an understanding of how this insurance plan works. LRP is designed to provide producers downside price risk protection. It is also important to distinguish between two types of cattle for which this insurance protects, i.e. feeder or fed cattle.

\subsubsection{LRP for Feeder Cattle}

Feeder cattle are those within a weight range of 650-850 lbs that are destined for finishing in a feedlot. They are back-grounded on forage or a grain fed diet until they are transported to feedlots or slaughtered. LRP insurance payment or indemnity payments are based on an insured coverage price available at the RMA web site and the actual ending value reflected in the Chicago Mercantile Exchange (CME) feeder Cattle Cash Price Index (CPI). This CPI represents the 7-day weighted average price of 650-850 lb. steers in a 12 state area $(\mathrm{CO}, \mathrm{IA}, \mathrm{KS}, \mathrm{MO}, \mathrm{MT}$, NE, NM, ND, OK, SD, TX and WY) (cmegroup.com/trading/commodities/). This CPI is again the same index which is used to cash-settle open CME feeder cattle contracts at expiration. It is believed that this index will generally be very close to CME feeder cattle futures prices. So, in effect, purchasing a LRP policy for feeder cattle is equivalent to buying an insurance policy that pays indemnity protection if the CME feeder cattle cash index (or the CPI) falls below the 
insured coverage price. This insured coverage price is based on the expected ending value at the end of the policy as determined at the onset. It results in "nationalizing" the sale price of cattle as the indemnities are based on changes in the feeder cattle cash index, rather than actual changes at the local stockyard (e.g. local cattle auction markets) (McConnell, 2007). It is possible that the local market could drop the overall price, but the CME index stays constant since it is the average of the 12 market price. This is the way it offers price risk protection. However, it is generally observed that the local cattle market and the CME feeder cattle cash index travel in tandem. It is also assumed that the fundamental factors that affect cattle prices in one market should similarly affect cattle in the overall market represented by the CME Feeder Cattle Cash Index (i.e., the 12 state indexes as mentioned earlier). For example, when corn prices increase, downward pressure will occur on feeder cattle prices across all states, as corn is one of the main ingredients in cattle grain feed. However, after consulting with beef experts in the local region (Dr. Evans and Dr. Rayburn), it was found that there could be times when prices in West Virginia (WV) do not move perfectly with western cattle prices represented by the index. In an informal e-mail based survey it was found that, during the past few years local pasture beef producers had endured consecutive droughts. This may increase supply in WV markets as lack of forage pressurizes producers to sell their cattle early, causing downward pressure on local prices, while those from other regions could remain unaffected, given the relatively small size of the WV cattle market relative to the national market.

\subsubsection{A Simple Example}

Let us consider a producer who has purchased an insurance policy with a coverage price of $\$ 105$ per hundredweight (cwt). The producer would pay an insurance premium for this policy on the day it becomes effective, and the policy would remain in place until the specified ending date. If the CME feeder cattle cash index price was above $\$ 105$ per cwt on its ending day, then the 
producer would not receive an indemnity payment. If the index is below $\$ 105$ per cwt on the ending date, then the producer would receive an indemnity for the difference. If the CME Feeder Cattle Cash Index Price was $\$ 102$ on the expiration date and the producer had insured 700 pound calves, then the indemnity payment would be $\$ 21$ per calf [7 cwt*(\$105-\$102)].

\subsubsection{Fed Cattle}

\section{Actual Ending Value-Fed Cattle}

The price of fed cattle that was used is that calculated by the Agricultural Marketing Service (AMS). This report is released once a week. It is based on 5 area (Texas/Oklahoma/New Mexico; Kansas; Nebraska; Colorado; Iowa/Minnesota) market summary. The report used to calculate the actual ending value will be the report from the week that contains the end date for the endorsement. If there is no reported information, then the calculation will be based on the latest report made prior to the end date. This report/price will be refereed as AMS 5 Area market price later in this essay.

\subsubsection{How to Purchase LRP?}

LRP insurance can be purchased through licensed crop insurance agents. The first step is to fill out the necessary enrollment form. The next step is to fill out a "Specific Coverage Endorsement" form on the day the producer wants the policy to become effective. Eligibility requirements are fairly simple. Cattle must be held in the state that is specified by the insurance policy. Purchasers of insurance are also required to have at least $10 \%$ ownership in the cattle (and they can only insure their interest in the cattle). This product is not intended for speculation, so the purchaser could be asked to provide proof of ownership when purchasing the coverage at a later date. The minimum contract length, referred to as the endorsement length for LRP coverage, is approximately thirteen weeks (rma.gov). Thus, the LRP cannot be used to manage short term price risk due to a limited time period of less than 13 weeks. Ending dates are 
available in four-week increments, although the availability of an ending date depends on the exchange traded option volume for deferred contracts traded on the CME that day.

LRP insurance is available for steers and heifers of different weights as well as different breed-types. Adjustments are made for lighter steers and heifers, as well as dairy-type and Brahman calves as shown in Table1.1. This adjustment is made in order to bring the coverage price closer to the actual cash value of the cattle.

Table 1-1.: Adjustments in CME Feeder Cattle Index for Cattle Size and Type

\begin{tabular}{|l|l|l|l|l|}
\hline Category & Steers & Heifers & Dairy & Brahman \\
\hline Light: Less than 600 lbs & $110 \%$ & $100 \%$ & $85 \%$ & $100 \%$ \\
\hline Heavy: 600 lbs to 900 lbs & $100 \%$ & $90 \%$ & $80 \%$ & $90 \%$ \\
\hline
\end{tabular}

(Data Source: rma.usda.gov/livestock/)

\subsection{LRP Versus Cattle Put Options}

Buying insurance coverage is similar to buying a put option. A put option is a financial contract that gives the holder the right to sell. A few very common, similar put options are car insurance or agricultural price supports. By purchasing car insurance, we actually buy a put option with the current market value of the car as the strike or exercise price. Similarly, an agricultural price support program is another example of a put option. In effect, the government actually writes a put option to farmers. If the market price goes down, farmers can sell the crop to the government at the set exercise or strike price; hence exercising the put option. If the market price for the crop is high, they do not need to exercise this option that the government has provided to them. Using a similar analogy, purchasing LRP insurance for feeder cattle is similar to purchasing a putoption at the CME, in that the coverage price works in a similar manner to a strike or exercise price. A put option contract moves into the money (i.e., makes profit) when the futures price falls below a strike price, resulting in a profit. This pay-off of a long put is described in section 3.8. Similarly, LRP insurance will pay an indemnity when the CME feeder cattle cash index is 
below a certain coverage level on a specified ending date. There are several advantages with the LRP program over futures trading as follows. First, producers already have a working relationship established with an insurance agent who is selling the LRP insurance. Second, a commission is not paid by the producer for LRP insurance, whereas it would be paid to the broker when purchasing a put-option. Finally, the LRP program allows coverage for smaller cattle operations. This is in contrast to futures/options contracts that must be purchased in increments of 50,000 lbs, a size too large for most grass-fed producers. The flexibility of the LRP program allows producers to cover a much smaller number of cattle.

A major disadvantage of the LRP program is that there is no flexibility in closing the contract. Once purchased, LRP insurance cannot be sold back, unlike futures contracts. LRP insurance is like buying a put-option that can only be exercised on the last day. That is it works like a European option. LRP is also less flexible with respect to the actual sale of the cattle. If the cattle are sold more than 30 days prior to the end date, the insurance becomes void. This presents a potential problem in years of drought, when producers need to sell earlier than originally planned. Cattle may be sold anytime after the end date, but the indemnity is still figured into the value of the corresponding end date. So, the producers still run the risk of prices dropping after the ending date but before the calves are sold.

\subsection{Empirical Model}

\subsubsection{Theory of Stochastic Dominance Analysis}

This analysis can be initiated by a simple assumption that an individual's choice between actions or alternatives is made such that the outcome of the choice will maximize their expected utility. Axiomatically, this assumption is based on Bernoulli's principle which was expanded upon by Von Neumann and Morgenstern. Mathematically, this principle is based on three axioms (Anderson, Dillon and Hardeker, p. 66-69): 
1. Ordering and transitivity: A person either prefers one of the two prospects $a_{1}$ and $a_{2}$ or is indifferent between them. Further, if the individual prefers $a_{1}$ to $a_{2}$ and $a_{2}$ to $a_{3}$ then he prefers $a_{1}$ to $a_{3}$.

2. Continuity: If a person prefers $a_{1}$ to $a_{2}$ to $a_{3}$, then there exists some subjective probability level $\operatorname{Pr}\left[a_{1}\right]$ such that $\mathrm{s} /$ he is indifferent between the gamble paying $a_{1}$ with probability $\operatorname{Pr}[$ $\left.a_{1}\right]$ and $a_{3}$ with probability 1-Pr $\left[a_{1}\right]$ which leaves him/her indifferent with $a_{2}$.

3. Independence: If $a_{1}$ is preferred to $a_{2}$ and $a_{3}$ is any other risky prospect, a lottery with $a_{1}$ and $a_{3}$ outcomes will be preferred to a lottery with $a_{2}$ and $a_{3}$ outcomes when $\operatorname{Pr}\left[a_{1}\right]=\operatorname{Pr}[$ $a_{2}$ ]. In other words, preference between $a_{1}$ and $a_{2}$ is independent of $a_{3}$.

There is some literature questioning these assumptions. For example, May (1954) and Tversky (1969) questioned the transitivity of preferences. These studies question whether preferences under uncertainty are adequately described by the traditional expected utility framework. One alternative is to develop risk efficiency criteria rather than expected utility axioms. Risk efficiency criteria are an attempt to reduce the collection of all possible alternatives to a smaller collection of risky alternatives that contain the optimum choice. One example is the mean-variance derivation of optimum portfolios. The efficient frontier, a result of mean-variance optimization, contains the set of possible portfolios, such that no other portfolio could be constructed with a higher return with the same risk (measured by the variance of that particular portfolio). It was the general contention that this efficient set contained the utility maximizing portfolio. Instead of expected utility justifying risk efficiency, we are interested in the derivation of risk efficiency measures per se. An alternative justification of 
risk efficiency measures involves the scenario where the individual's risk preferences are difficult to elicit.

The concept of efficiency criteria can be developed as follows. According to Meyer 1977, an efficiency criterion is a decision rule for dividing alternatives into two mutually exclusive groups: efficient and inefficient. If an alternative is in the efficient group, then it is one that an investor may choose. An inefficient investment will not be chosen by any investor regardless of individual risk preference. From an economic standpoint, the criteria should be related to general notions of utility or preferences. In general, the more global the preference, the less discerning the criteria (i.e. the fewer alternatives eliminated). A smaller efficient set requires more stringent requirements on preferences. One of the most frequently used risk efficiency approaches is analyzed using stochastic dominance. To demonstrate the concept of stochastic dominance, let's examine two forms of stochastic dominance: first and second order stochastic dominance rules (collectively FSD and SSD).

To develop first order stochastic dominance (Moss, 2005), let us assume that the decision maker is faced with two alternative investments, $a$ and $b$. Assume that the probability density function for alternative $a$ can be characterized by the probability density function $f(\mathrm{x})$. Similarly, assume that the return on investment $b$ is associated with the probability density function $g(x)$. Investment $a$ is said to be first order dominant over investment $b$ if and only if $\int_{-\infty}^{x} g(x) d x \geq \int_{-\infty}^{x} f(x) d x$ 


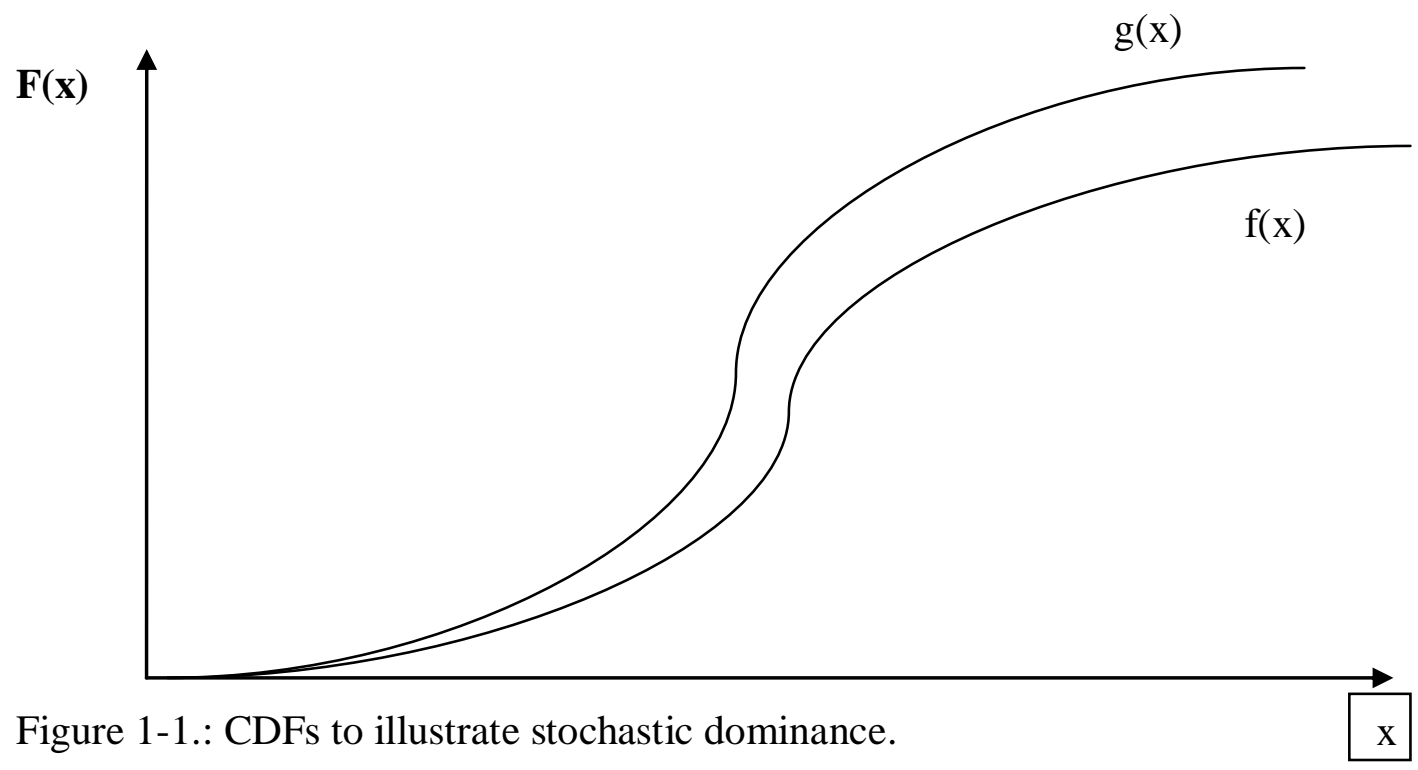

Thus, investment $a$ is always more likely to yield a higher return. Intuitively, one investment is going to dominate the other investment if their cumulative distribution functions do not cross. Economically, the only axiom required for first degree stochastic dominance is that the individual prefers more to less, or is non-satiated in consumption.

Mathematically, FSD is dependent on the integrals of the utility function times each alternative distribution function:

$E_{F} U(x)=\int_{a}^{b} U(x) f(x) d x$ Against $E_{G} U(x)=\int_{a}^{b} U(x) g(x) d x$

Note that the utility function is the same for each investment alternative, but the distribution function changes. If investment $\mathrm{F}$ dominates investment $\mathrm{G}$, then the investment, $\Delta$, defined as

$\Delta=E_{F} U(x)-E_{G} U(x)=\int_{a}^{b}[f(x)-g(x)] U(x) d x$

must be greater than or equal to zero for all $\mathrm{x}$ with at least one strict inequality.

\subsubsection{Second Degree Stochastic Dominance}

As noted in the preceding section, the only requirement for FSD to be valid is that the decision maker prefers more to less. Building on this concept, second order stochastic dominance (SSD) invokes risk aversion by inferring that the utility function is concave, implying that the second derivative of the utility function is negative. 
The SSD rule can be described as follows. A necessary and sufficient condition for an alternative $\mathrm{F}$ to be preferred to a second alternative $\mathrm{G}$, by all risk adverse decision makers is that $\int_{-\infty}^{x} F(z) d z \leq \int_{-\infty}^{x} G(z) d z$ For all $\mathrm{x}$, or $\int_{-\infty}^{x}[G(z)-F(z)] d z \geq 0$ With at least one inequality. Mathematically, building on first degree stochastic dominance:

$$
\Delta=\int_{a}^{b}[G(x)-F(x)] U^{\prime}(x) d x
$$

Which is positive if $\int_{a}^{x}[G(z)-F(z)] d z \leq 0$ for all $\mathrm{x}$ with a strict inequality for at least one $\mathrm{x}$.

The theory of utility, expected utility, risk preferences, and decision making under uncertainty in general, upon which the analysis in this chapter is based, has a rich heritage in the applied economics profession, as evidenced by the following works: Borch (1968); Tisdell (1968); Hey (1979); Karni (1985); and Laffont (1989).

\subsection{The Empirical Model}

An empirical model was developed to measure the price risk associated with grass-fed beef production and also to evaluate how these producers would respond to the LRP of USDA/RMA. The distribution of payoffs from this model was evaluated using FSD or First Degree Stochastic Dominance Rule. There are quite a few empirical studies which show that the properties of FSD and SSD as described in previous sections hold for most individuals (Anderson, 1974; Antle, 1987; Chavas and Holt, 1996). Similarly, stochastic dominance (SD) analysis has been used in a wide variety of farm studies. Some of them, including Hien et al. (1997) used SD analysis to estimate the riskiness of alternative phosphate sources for African farmers. Hallman and Lowenberg-DeBoer (1999) used SD techniques to assess the economic advantages of switching to narrow row corn. There are also other studies (e.g. Ribera, Hons and Richardson 2004; Sangtaek, Mitchell and Leatham 2005) which build upon using stochastic dominance to evaluate risks and cropping decisions. 
The model of this study can be described as:

Net Return = Net Revenue + Indemnity Paid Out - Premium Paid + Production Cost (5);

We are omitting the production cost from our study as we assume that it is fixed or constant across all the cases. Hence our model reduces to:

Net Return = Net Revenue + Indemnity Paid Out - Premium Paid (6);

Net Revenue is calculated from the parent project data base of the Pasture-beef project consortium. This data base maintains record of cattle finished at the Beaver project trial center of the USDA Agriculture Research Service (ARS). Currently this data base consists of the last 5 years of pasture raised cattle data (of about 200 animals; since 2004). The software @ Risk was used to get the best fit distribution of the weights of these animals. A Monte Carlo simulation was carried out to generate 1,000 observations using the best fit distribution reported by @ Risk. Beef revenue was then calculated by using the West Virginia sales price as applicable in October 2008 from the USDA National Agriculture Statistics Service (NASS). These steps are presented in the flow chart of figure 1.7. Indemnity is calculated by:

Rounded Total Premium $=(\#$ of head $*$ target weight $*$ coverage price $*$ insured share $*$ rate $)(7)$;

USDA/RMA subsidizes the premium by $13 \%$, so the actual producer premium is:

Producer Premium $=($ Rounded Total Premium *0.87) $(8)$;

The producer premium is calculated by:

Indemnity $=(\#$ of head $*$ target weight $*($ coverage price-ending value $) *$ insured share $)(9)$; 


\subsection{Model Assumptions}

Table 1-2.: Model Assumptions for Essay I

\begin{tabular}{|c|c|}
\hline $\begin{array}{c}\text { Model } \\
\text { Parameters }\end{array}$ & Description \\
\hline Shipping Date & $\begin{array}{l}\text { After consulting with industry experts and practioners, the optimal cattle } \\
\text { shipping date has been fixed as } 15 \text { th September, i.e., } 09 / 15 / 2008 \text {. }\end{array}$ \\
\hline Model & Net Return $=$ Net Sale Price + Indemnity Received-Premiums Paid \\
\hline $\begin{array}{l}\text { Type of Costs } \\
\text { Considered }\end{array}$ & $\begin{array}{l}\text { We have eliminated production costs from the model and only included the } \\
\text { cost of the insurance premium. }\end{array}$ \\
\hline $\begin{array}{l}\text { Best Fit } \\
\text { Distribution }\end{array}$ & $\begin{array}{l}\text { Beta General }(11.939,91.517,313.1423) \text { as reported by @ Risk. Numbers in } \\
\text { parenthesis indicate the distribution parameters of the finish weight. }\end{array}$ \\
\hline $\begin{array}{l}\text { Coverage } \\
\text { Lengths }\end{array}$ & 17 different choices with minimum length of 13 to maximum 26 weeks. \\
\hline Price Scenarios & $\begin{array}{l}\text { Scenarios: Base Price; a 5\% discount; and premiums of 5\%, } 10 \%, 15 \% \text { and } \\
20 \% \text {. It was important to generate these price scenarios as the direct } \\
\text { marketers' charge a premium or discount over the market beef price as found } \\
\text { in an earlier survey conducted by WVU Extension dept. This survey is located } \\
\text { at: } \\
\text { http://www.caf.wvu.edu/ forage/PFBSurvey.pdf. These price scenarios also } \\
\text { deemed to be exact by Dr. Jason Evans, SUNY who has worked extensively } \\
\text { in grass fed production systems. }\end{array}$ \\
\hline Case Studies & $\begin{array}{l}\text { A: Indemnity, paid as of } 09 / 15 / 08 \text {. } \\
\text { B: Selecting two end values after examining the actual daily LRP end values } \\
\text { of } 15^{\text {th }} \text { Sep- } 15^{\text {th }} \text { Oct, } 2005-2009 \text {. }\end{array}$ \\
\hline
\end{tabular}




\section{Methodology flowchart}

Use @Risk best fit to determine the best fit distribution of animal weight.

Carry out Monte Carlo Simulation to generate 1,000 observations using the distribution reported by Best Fit.

Calculate beef revenue using West Virginia Beef Price by cwt

Generate 5 more price scenarios for a $5 \%$ discount; and price premiums of $5 \% .10 \% .15 \%$ and $20 \%$

Generate 17 net return distributions for each price and analyze the stochastic dominance using Simetar.

Figure 1-2.: Methodology Flowchart of Essay I.

Stochastic dominance analysis was carried out using Simetar software [http://simetar.com/]. It is an Excel add-in, developed at Texas A\&M University. This is a simple but powerful tool to conduct risk analysis. Once the net return scenarios are compiled, these can be inputted into Simetar to build up the cumulative distribution functions (CDFs The risk rankings are also reported. Simetar makes use of exponential and power utility functions to generate stochastic dominance with respect to a function (SDRF) CDFs. Use of an exponential utility function gives rise to a constant absolute risk aversion coefficient (CARA), whereas use of a power utility function gives rise to a constant relative risk aversion coefficient (CRRA).

Table 1-3 shows the 17 possible choices and table 1-4 shows the associated return scenarios

for these

17

risky

outcomes. 
Table 1-3.: Various LRP Insurance choices with 10/15/2008 as the end date

\begin{tabular}{|c|c|c|c|c|c|c|c|c|c|c|}
\hline Choice(s) & State & County & $\begin{array}{l}\text { Endorsement } \\
\text { Length }\end{array}$ & $\begin{array}{l}\text { Commo } \\
\text { dity }\end{array}$ & Type & $\begin{array}{l}\text { Exp. End } \\
\text { Value } \\
(\$)\end{array}$ & $\begin{array}{l}\text { Coverage } \\
\text { Price } \\
(\$)\end{array}$ & $\begin{array}{l}\text { Coverage } \\
\text { Level }\end{array}$ & End Date & $\begin{array}{l}\text { Actual End } \\
\text { Value } \\
\text { (\$) }\end{array}$ \\
\hline I. & WV & ALL & 13weeks & $\begin{array}{l}0802 \\
\text { Fed } \\
\text { Cattle }\end{array}$ & $\begin{array}{l}\text { Steer } \\
\text { /heifers }\end{array}$ & 105.807 & 91.23 & 0.8622 & $09 / 15 / 08$ & 98.59 \\
\hline II. & WV & ALL & 13 weeks & $\begin{array}{l}0802 \\
\text { Fed } \\
\text { Cattle }\end{array}$ & , & 105.807 & 87.23 & 0.8244 & $09 / 15 / 08$ & 98.59 \\
\hline III. & WV & ALL & 13 weeks & $\begin{array}{l}0802 \\
\text { Fed } \\
\text { Cattle }\end{array}$ & , & 105.807 & 81.23 & 0.7677 & $09 / 15 / 08$ & 98.59 \\
\hline IV. & WV & ALL & 13 weeks & $\begin{array}{l}0802 \\
\text { Fed } \\
\text { Cattle }\end{array}$ & , & 105.807 & 79.23 & 0.7488 & $09 / 15 / 08$ & 98.59 \\
\hline V. & WV & ALL & 17 weeks & $\begin{array}{l}0802 \\
\text { Fed } \\
\text { Cattle }\end{array}$ & , & 99.956 & 93.66 & 0.937 & $09 / 15 / 08$ & 98.59 \\
\hline VI. & WV & ALL & 17 weeks & $\begin{array}{l}0802 \\
\text { Fed } \\
\text { Cattle }\end{array}$ & , & 99.956 & 91.66 & 0.917 & $09 / 15 / 08$ & 98.59 \\
\hline VII. & WV & ALL & 17 weeks & $\begin{array}{l}0802 \\
\text { Fed } \\
\text { Cattle }\end{array}$ & , & 99.956 & 89.66 & 0.897 & $09 / 15 / 08$ & 98.59 \\
\hline VIII. & WV & ALL & 17 weeks & $\begin{array}{l}0802 \\
\text { Fed } \\
\text { Cattle }\end{array}$ & , & 99.956 & 87.66 & 0.877 & $09 / 15 / 08$ & 98.59 \\
\hline IX. & WV & ALL & 17 weeks & $\begin{array}{l}0802 \\
\text { Fed } \\
\text { Cattle }\end{array}$ & , & 99.956 & 81.66 & 0.817 & $09 / 15 / 08$ & 98.59 \\
\hline $\mathrm{X}$. & WV & ALL & 21 weeks & $\begin{array}{l}0802 \\
\text { Fed }\end{array}$ & , & 98.994 & 94.34 & 0.953 & $09 / 15 / 08$ & 98.59 \\
\hline
\end{tabular}




\begin{tabular}{|c|c|c|c|c|c|c|c|c|c|c|}
\hline & & & & Cattle & & & & & & \\
\hline Choice(s) & State & County & $\begin{array}{l}\text { Endorsement } \\
\text { Length }\end{array}$ & $\begin{array}{l}\text { Commo } \\
\text { dity }\end{array}$ & Type & $\begin{array}{l}\text { Exp. End } \\
\text { Value } \\
(\$)\end{array}$ & $\begin{array}{l}\text { Coverage } \\
\text { Price } \\
(\$) \\
\end{array}$ & $\begin{array}{l}\text { Coverage } \\
\text { Level }\end{array}$ & End Date & $\begin{array}{l}\text { Actual End } \\
\text { Value } \\
(\$)\end{array}$ \\
\hline XI. & WV & ALL & 21 weeks & $\begin{array}{l}0802 \\
\text { Fed } \\
\text { Cattle } \\
\end{array}$ & " & 98.994 & 92.34 & 0.9328 & $09 / 15 / 08$ & 98.59 \\
\hline XII. & WV & ALL & 21 weeks & $\begin{array}{l}0802 \\
\text { Fed } \\
\text { Cattle } \\
\end{array}$ & " & 98.994 & 90.34 & 0.9126 & $09 / 15 / 08$ & 98.59 \\
\hline XIII. & WV & ALL & 21 weeks & $\begin{array}{l}0802 \\
\text { Fed } \\
\text { Cattle } \\
\end{array}$ & " & 98.994 & 86.34 & 0.8722 & $09 / 15 / 08$ & 98.59 \\
\hline XIV. & WV & $\overline{A L L}$ & 26 weeks & $\begin{array}{l}0802 \\
\text { Fed } \\
\text { Cattle } \\
\end{array}$ & " & 95.341 & 93.97 & 0.9856 & $09 / 15 / 08$ & 98.59 \\
\hline $\mathrm{XV}$. & WV & ALL & 26 weeks & $\begin{array}{l}0802 \\
\text { Fed } \\
\text { Cattle } \\
\end{array}$ & , & 95.341 & 87.97 & 0.9227 & $09 / 15 / 08$ & 98.59 \\
\hline XVI. & WV & ALL & 26 weeks & $\begin{array}{l}0802 \\
\text { Fed } \\
\text { Cattle }\end{array}$ & ", & 95.341 & 81.97 & 0.8588 & $09 / 15 / 08$ & 98.59 \\
\hline XVII. & & & & & & NSUR & & & & \\
\hline
\end{tabular}


Table 1-4.: Various Return Scenarios

\begin{tabular}{|c|c|c|c|c|}
\hline Choice & Coverage Price (\$per cwt) & Coverage Level & Premium without Subsidy (\$ per cwt) & Premium with subsidy (\$ per cwt) \\
\hline I. & & & 0.538 & 0.24 \\
\hline II. & 91.23 & 0.8622 & 0.279 & 0.24 \\
\hline III. & 81.23 & 0.8244 & 0.094 & 0.081 \\
\hline IV. & 79.23 & 0.7677 & 0.060 & 2.18 \\
\hline V. & 93.66 & 0.937 & 2.510 & 1.58 \\
\hline VI. & 91.66 & 0.917 & 1.823 & 1.10 \\
\hline VII. & 89.66 & 0.897 & 1.268 & 0.75 \\
\hline VIII. & 87.66 & 0.877 & 0.865 & 0.21 \\
\hline IX. & 81.66 & 0.953 & 0.242 & 2.87 \\
\hline X. & 94.34 & 0.9328 & 3.297 & 2.12 \\
\hline XI. & 92.34 & 0.9126 & 2.437 & 1.55 \\
\hline XII. & 90.34 & 0.8722 & 1.781 & 0.79 \\
\hline XIII. & 86.34 & 0.9856 & 0.911 & 4.09 \\
\hline XIV. & 93.97 & 0.9227 & 4.699 & 1.79 \\
\hline XV. & 87.97 & 0.8588 & 2.066 & 0.64 \\
\hline XVI. & 81.97 & 0.8622 & 0.736 & 0 \\
\hline XVII. & 91.23 & & & \\
\hline
\end{tabular}




\subsection{Data Sources}

Data used in the simulation was obtained from the parent project database. The following table explains the different data sources used in this study.

Table 1-5.: Data Sources for essay I

\begin{tabular}{|l|l|}
\hline Variable & Data Source \\
\hline $\begin{array}{l}\text { Hard Carcass Weight } \\
\text { /Cattle Weight }\end{array}$ & Project Data base \\
\hline Beef Revenue & $\begin{array}{l}\text { West Virginia Beef price by cwt [USDA-WV market news] and } \\
\text { NASS } \\
\text { Price as pertinent of 08.15.2008. }\end{array}$ \\
\hline $\begin{array}{l}\text { LRP } \\
\text { Premium/Indemnity }\end{array}$ & $\begin{array}{l}\text { USDA, AMS } \\
\text { http://www3.rma.usda.gov/apps/livestock_reports/main.aspx }\end{array}$ \\
\hline Basis Risk & $\begin{array}{l}\text { National Agricultural Statistics Service (NASS) and USDA- } \\
\text { PA/USDA-VA Dept of Agriculture Market News }\end{array}$ \\
\hline
\end{tabular}

\subsection{Basis in Local Cattle Market}

Basis refers to the difference in prices realized in different markets with regard to risk. The main purpose of LRP is to protect producers with the price differential between the local and national market. WV grass fed producers typically sell their cattle at cattle auction markets in Virginia (located in Winchester) and Pennsylvania (located in New Holland, Lancaster). It was important to examine if any basis exists between the auction markets in VA/PA and AMS 5 market prices. The graph below shows the historical basis that exists in these three markets. Data was collected from the National Agricultural Statistics Service (NASS) and USDA-PA/USDA-VA Dept of Agriculture Market News. 


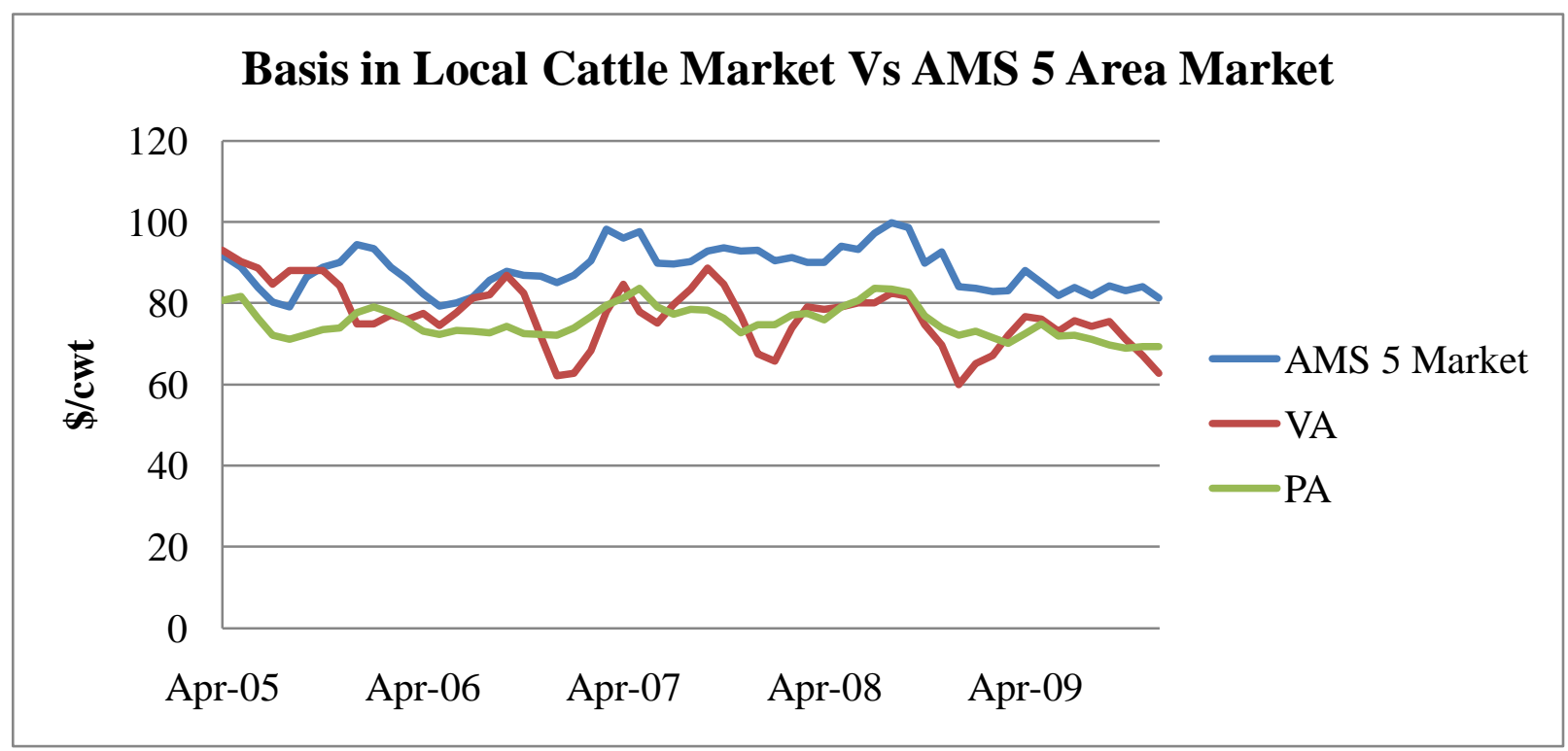

Figure 1-9.: Basis in Local Cattle Market (Data Source: NASS/Market News).

From the above graph, it can be seen that indeed a basis exists, between the local cattle and the AMS 5 area markets. It can also be seen that, on average, there is a basis of $\$ 11.21$ for the VA/Winchester auction market and \$13.01 for the PA/Lancaster auction market based on the last 5 years of NASS data.

\subsection{Estimation and Results}

After studying the existing basis, stochastic dominance analysis was subsequently used to analyze 17 scenarios of the model described earlier. In addition, two case studies were created, as follows: Case A is actual indemnities/premium as paid out as of 09/15/08. For Case B I chose two actual end values after examining 5 years of actual end values for LRP which are $\$ 84$ and $\$ 89$ respectively.. 


\section{Case A: Actual End Value as of 09/15/2008 (Actual End Value: \$98.59)}

Table 1-6.: Descriptive Statistics of Net Return Distributions of 17 different insurance choices with Actual End Value as of 09/15/2008 (Values in \$).

Base Price Scenario:

\begin{tabular}{|c|c|c|c|c|c|c|c|c|c|c|c|c|c|c|c|c|c|}
\hline Type & I & II & III & IV & $\mathrm{V}$ & VI & VII & VIII & IX & $\mathrm{X}$ & XI & XII & XII & XIV & $\mathrm{XV}$ & XVI & XVII \\
\hline Mean & 1390.94 & 1393.19 & 1394.80 & 1395.10 & 1373.78 & 1379.76 & 1384.59 & 1388.09 & 1393.51 & 1366.94 & 1374.11 & 1380.13 & 1387.69 & 1354.74 & 1377.65 & 1389.22 & 1395.62 \\
\hline Std.Dev & 183.46 & 183.46 & 183.46 & 183.46 & 183.46 & 183.46 & 183.46 & 183.46 & 183.46 & 183.46 & 183.46 & 183.46 & 183.46 & 183.46 & 183.46 & 183.46 & 183.45 \\
\hline Min. & 917.02 & 919.27 & 920.88 & 921.18 & 899.86 & 905.84 & 910.67 & 914.17 & 919.59 & 893.02 & 900.18 & 906.20 & 913.77 & 880.82 & 903.73 & 915.30 & 921.70 \\
\hline Max. & 2012.27 & 2014.53 & 2016.14 & 2016.43 & 1995.12 & 2001.09 & 2005.92 & 2009.43 & 2014.85 & 1988.27 & 1995.44 & 2001.46 & 2009.03 & 1976.07 & 1998.98 & 2010.55 & 2016.95 \\
\hline
\end{tabular}

\section{Discounted Price Scenario:}

\begin{tabular}{|c|c|c|c|c|c|c|c|c|c|c|c|c|c|c|c|c|c|}
\hline Type & I & II & III & IV & $\mathrm{V}$ & VI & VII & VIII & IX & $X$ & XI & XII & XII & XIV & $\mathrm{XV}$ & XVI & XVII \\
\hline Mean & 1321.16 & 1323.41 & 1325.02 & 1325.32 & 1304.00 & 1309.98 & 1314.81 & 1318.31 & 1323.73 & 1297.16 & 1304.32 & 1310.34 & 1317.91 & 1284.96 & 1307.87 & 1321.16 & 1325.84 \\
\hline Std.Dev & 174.28 & 174.28 & 174.28 & 174.28 & 174.28 & 174.28 & 174.28 & 174.28 & 174.28 & 174.28 & 174.28 & 174.28 & 174.28 & 174.28 & 174.28 & 174.28 & 174.28 \\
\hline Min. & 870.93 & 873.19 & 874.80 & 875.09 & 853.78 & 859.75 & 864.58 & 868.09 & 873.51 & 846.93 & 854.10 & 860.12 & 867.69 & 834.73 & 857.64 & 870.93 & 875.61 \\
\hline
\end{tabular}




\begin{tabular}{|c|c|c|c|c|c|c|c|c|c|c|c|c|c|c|c|c|c|}
\hline Type & I & II & III & IV & $\mathrm{V}$ & VI & VII & VIII & IX & $\mathrm{X}$ & XI & XII & XII & XIV & XV & XVI & XVII \\
\hline Max. & 1911.43 & 1913.68 & 1915.29 & 1915.58 & 1894.27 & 1900.25 & 1905.07 & 1908.58 & 1914.00 & 1887.42 & 1894.59 & 1900.61 & 1908.18 & 1875.23 & 1898.13 & 1911.43 & 1916.11 \\
\hline
\end{tabular}

\section{5\% Premium}

\begin{tabular}{|c|c|c|c|c|c|c|c|c|c|c|c|c|c|c|c|c|c|}
\hline Type & $T$ & II & III & IV & V & VI & VII & VIII & TX & $X$ & $\mathrm{XI}$ & $\mathrm{XII}$ & $\mathrm{XII}$ & XIV & $\mathrm{XV}$ & $\mathrm{XVI}$ & XVII \\
\hline Mean & 1321.16 & 1323.41 & 1254.87 & 1257.12 & 1258.73 & 1259.03 & 1237.71 & 1243.69 & 1248.52 & 1252.02 & 1257.44 & 1254.87 & 1257.12 & 1258.73 & 1259.03 & 1237.71 & 1259.55 \\
\hline Std.Dev & 174.28 & 174.28 & 165.57 & 165.57 & 165.57 & 165.57 & 165.57 & 165.57 & 165.57 & 165.57 & 165.57 & 165.57 & 165.57 & 165.57 & 165.57 & 165.57 & 165.57 \\
\hline Min. & 870.93 & 873.19 & 827.15 & 829.41 & 831.02 & 831.31 & 810.00 & 815.97 & 820.80 & 824.31 & 829.73 & 827.15 & 829.41 & 831.02 & 831.31 & 810.00 & 831.83 \\
\hline Max. & 1911.43 & 1913.68 & 1815.62 & 1817.87 & 1819.48 & 1819.78 & 1798.46 & 1804.44 & 1809.27 & 1812.77 & 1818.19 & 1815.62 & 1817.87 & 1819.48 & 1819.78 & 1798.46 & 1820.30 \\
\hline
\end{tabular}

\section{0\% Premium}

\begin{tabular}{|c|c|c|c|c|c|c|c|c|c|c|c|c|c|c|c|c|c|}
\hline Type & 1 & II & III & IV & V & VI & VII & VIII & IX & $X$ & $\mathrm{XI}$ & XII & XII & XIV & $X V$ & XVI & XVII \\
\hline Mean & 1191.89 & 1194.14 & 1195.75 & 1196.05 & 1174.73 & 1180.71 & 1185.54 & 1189.04 & 1194.46 & 1167.89 & 1175.06 & 1181.08 & 1191.89 & 1194.14 & 1195.75 & 1196.05 & 1196.57 \\
\hline Std.Dev & 157.29 & 157.29 & 157.29 & 157.29 & 157.29 & 157.29 & 157.29 & 157.29 & 157.29 & 157.29 & 157.29 & 157.29 & 157.29 & 157.29 & 157.29 & 157.29 & 157.29 \\
\hline Min. & 785.56 & 787.81 & 789.42 & 789.72 & 768.40 & 774.38 & 779.21 & 782.72 & 788.14 & 761.56 & 768.73 & 774.75 & 785.56 & 787.81 & 789.42 & 789.72 & 790.24 \\
\hline
\end{tabular}




\begin{tabular}{|c|c|c|c|c|c|c|c|c|c|c|c|c|c|c|c|c|c|}
\hline Type & $T$ & III & III & IV & $V$ & VI & VII & VIII & IX & $X$ & $\mathrm{XI}$ & XII & XII & XIV & $X V$ & XVI & XVII \\
\hline Max. & 1724.61 & 1726.86 & 1728.47 & 1728.76 & 1707.45 & 1713.43 & 1718.25 & 1721.76 & 1727.18 & 1700.60 & 1707.77 & $1713.7 \quad 9$ & 1724.61 & 1726.86 & 1728.47 & 1728.76 & 1729.29 \\
\hline
\end{tabular}

\section{5\% Premium}

\begin{tabular}{|c|c|c|c|c|c|c|c|c|c|c|c|c|c|c|c|c|c|}
\hline Type & 1 & III & III & IV & V & $\mathrm{VI}$ & VII & VIII & IX & $\mathrm{X}$ & $\mathrm{XI}$ & $\mathrm{XII}$ & XII & XIV & $X V$ & $\mathrm{XVI}$ & $\mathrm{XVII}$ \\
\hline Mean & 1132.06 & 1134.31 & 1135.92 & 1136.22 & 1114.90 & 1120.88 & 1125.71 & 1129.22 & 1134.64 & 1108.06 & 1115.23 & 1121.25 & 1132.06 & 1134.31 & 1135.92 & 1136.22 & 1136.74 \\
\hline Std.Dev & 149.43 & 149.43 & 149.43 & 149.43 & 149.43 & 149.43 & 149.43 & 149.43 & 149.43 & 149.43 & 149.43 & 149.43 & 149.43 & 149.43 & 149.43 & 149.43 & 149.43 \\
\hline Min. & 746.05 & 748.30 & 749.91 & 750.21 & 728.89 & 734.87 & 739.70 & 743.20 & 748.62 & 722.05 & 729.21 & 735.24 & 746.05 & 748.30 & 749.91 & 750.21 & 750.73 \\
\hline Max. & 1638.14 & 1640.39 & 1642.00 & 1642.30 & 1620.98 & 1626.96 & 1631.79 & 1635.30 & 1640.72 & 1614.14 & 1621.31 & 1627.33 & 1638.14 & 1640.39 & 1642.00 & 1642.30 & 1642.82 \\
\hline
\end{tabular}

\section{0\% Premium}

\begin{tabular}{|c|c|c|c|c|c|c|c|c|c|c|c|c|c|c|c|c|c|}
\hline Type & 1 & III & III & IV & V & VI & VII & VIII & IX & $X$ & $\mathrm{XI}$ & $\mathrm{XII}$ & XII & XIV & $X V$ & $\mathrm{XVI}$ & XVII \\
\hline Mean & 1068.87 & 1072.38 & 1077.80 & 1051.22 & 1058.39 & 1064.41 & 1071.98 & 1039.02 & 1061.93 & 1073.50 & 1068.87 & 1072.38 & 1077.80 & 1051.22 & 1058.39 & 1064.41 & 1079.90 \\
\hline Std.Dev & 141.96 & 141.96 & 141.96 & 141.96 & 141.96 & 141.96 & 141.96 & 141.96 & 141.96 & 141.96 & 141.96 & 141.96 & 141.96 & 141.96 & 141.96 & 141.96 & 141.96 \\
\hline
\end{tabular}




\begin{tabular}{|c|c|c|c|c|c|c|c|c|c|c|c|c|c|c|c|c|c|}
\hline Type & $\mathrm{T}$ & III & IIII & TV & V & VI & VII & VIII & IX & $X$ & $\mathrm{XI}$ & XII & XII & XIV & $X V$ & XVI & XVII \\
\hline Min. & 702.16 & 705.67 & 711.09 & 684.51 & 691.68 & 697.70 & 705.27 & 672.31 & 695.22 & 706.79 & 702.16 & 705.67 & 711.09 & 684.51 & 691.68 & 697.70 & 713.19 \\
\hline Max. & 1549.65 & 1553.15 & 1558.57 & 1532.00 & 1539.17 & 1545.19 & 1552.75 & 1519.80 & 1542.71 & 1554.28 & 1549.65 & 1553.15 & 1558.57 & 1532.00 & 1539.17 & 1545.19 & 1542.78 \\
\hline
\end{tabular}

\section{Case B: Introducing Randomness in Actual End Value}

Case B introduces some randomness into the calculation by changing the actual end value. After close inspection of AMS price reporting, the actual end value has a range between $\$ 84.11$ per cwt and $\$ 89.79$ per cwt; indemnity will be paid out in both cases, depending on insurance choices. The actual end values of LRP between $15^{\text {th }}$ Sept. to $15^{\text {th }}$ Oct., 2005-2009 are reported in Appendix D.

Table 1-7.: Descriptive Statistics of Net Distribution Returns (End Value: \$84.11). All Values are in \$.

Base Price Scenario:

\begin{tabular}{|c|c|c|c|c|c|c|c|c|c|c|c|c|c|c|c|c|c|}
\hline Type & 1 & III & III & TV & V & $\mathrm{VI}$ & VII & VIII & IX & $X$ & XI & XII & XII & XIV & $X V$ & XVI & XVII \\
\hline Mean & 1452.14 & 1406.39 & 1394.80 & 1395.10 & 1469.28 & 1437.26 & 1422.09 & 1405.59 & 1393.51 & 1451.24 & 1438.41 & 1424.43 & 1391.99 & 1435.34 & 1398.25 & 1452.14 & 1395.62 \\
\hline Std.Dev & 183.46 & 183.46 & 183.46 & 183.46 & 183.46 & 183.46 & 183.46 & 183.46 & 183.46 & 183.46 & 183.46 & 183.46 & 183.46 & 183.46 & 183.46 & 183.46 & 183.46 \\
\hline Min. & 978.22 & 932.47 & 920.88 & 921.18 & 995.36 & 963.34 & 948.17 & 931.67 & 919.59 & 977.32 & 964.48 & 950.50 & 918.07 & 961.42 & 924.33 & 978.22 & 921.70 \\
\hline Max. & 2073.47 & 2027.73 & 2016.14 & 2016.43 & 2090.62 & 2058.59 & 2043.42 & 2026.93 & 2014.85 & 2072.57 & 2059.74 & 2045.76 & 2013.33 & 2056.67 & 2019.58 & 2073.47 & 2016.95 \\
\hline
\end{tabular}




\section{Discounted Price Scenario:}

\begin{tabular}{|c|c|c|c|c|c|c|c|c|c|c|c|c|c|c|c|c|c|}
\hline Type & 1 & II & III & IV & V & $\mathrm{VI}$ & VII & VIII & IX & $X$ & $\mathrm{XI}$ & XII & $\mathrm{XII}$ & XIV & $X V$ & $\mathrm{XVI}$ & XVII \\
\hline Mean & 1374.36 & 1336.61 & 1325.02 & 1325.32 & 1381.50 & 1367.48 & 1352.31 & 1335.81 & 1323.73 & 1381.46 & 1368.62 & 1354.64 & 1322.21 & 1365.56 & 1328.47 & 1319.44 & 1325.84 \\
\hline Std.Dev & 174.28 & 174.28 & 174.28 & 174.28 & 174.28 & 174.28 & 174.28 & 174.28 & 174.28 & 174.28 & 174.28 & 174.28 & 174.28 & 174.28 & 174.28 & 174.28 & 174.28 \\
\hline Min. & 924.13 & 886.39 & 874.80 & 875.09 & 931.28 & 917.25 & 902.08 & 885.59 & 873.51 & 931.23 & 918.40 & 904.42 & 871.99 & 915.33 & 878.24 & 869.21 & 875.61 \\
\hline Max. & 1964.63 & 1926.88 & 1915.29 & 1915.58 & 1971.77 & 1957.75 & 1942.57 & 1926.08 & 1914.00 & 1971.72 & 1958.89 & 1944.91 & 1912.48 & 1955.83 & 1918.73 & 1909.70 & 1916.11 \\
\hline
\end{tabular}

5\% Premium

\begin{tabular}{|c|c|c|c|c|c|c|c|c|c|c|c|c|c|c|c|c|c|}
\hline Type & 1 & III & III & TV & V & $\mathrm{VI}$ & VII & VIII & TX & $X$ & $\mathrm{XI}$ & XII & $\mathrm{XII}$ & XIV & $\mathrm{XV}$ & XVI & XVII \\
\hline Mean & 1258.73 & 1259.03 & 1315.21 & 1301.19 & 1286.02 & 1269.52 & 1257.44 & 1315.16 & 1302.33 & 1288.35 & 1255.92 & 1299.27 & 1262.17 & 1253.14 & 1258.73 & 1259.03 & 1259.55 \\
\hline Std.Dev & 165.57 & 165.57 & 165.57 & 165.57 & 165.57 & 165.57 & 165.57 & 165.57 & 165.57 & 165.57 & 165.57 & 165.57 & 165.57 & 165.57 & 165.57 & 165.57 & 165.57 \\
\hline Min. & 831.02 & 831.31 & 887.50 & 873.47 & 858.30 & 841.81 & 829.73 & 887.45 & 874.62 & 860.64 & 828.21 & 871.55 & 834.46 & 825.43 & 831.02 & 831.31 & 831.83 \\
\hline Max. & 1819.48 & 1819.78 & 1875.96 & 1861.94 & 1846.77 & 1830.27 & 1818.19 & 1875.92 & 1863.09 & 1849.11 & 1816.68 & 1860.02 & 1822.93 & 1813.90 & 1819.48 & 1819.78 & 1820.30 \\
\hline
\end{tabular}

10\% Premium

\begin{tabular}{|c|c|c|c|c|c|c|c|c|c|c|c|c|c|c|c|c|c|}
\hline Type & $T$ & III & III & IV & V & $\mathrm{VI}$ & VII & VIII & IX & $X$ & $\mathrm{XI}$ & XII & XII & XIV & $\mathrm{XV}$ & $\mathrm{XVI}$ & XVII \\
\hline Mean & 1245.09 & 1207.34 & 1195.75 & 1196.05 & 1252.23 & 1238.21 & 1223.04 & 1206.54 & 1194.46 & 1252.19 & 1239.36 & 1225.38 & 1192.94 & 1236.29 & 1199.20 & 1190.17 & 1196.57 \\
\hline Std.Dev & 157.29 & 157.29 & 157.29 & 157.29 & 157.29 & 157.29 & 157.29 & 157.29 & 157.29 & 157.29 & 157.29 & 157.29 & 157.29 & 157.29 & 157.29 & 157.29 & 157.29 \\
\hline
\end{tabular}




\begin{tabular}{|c|c|c|c|c|c|c|c|c|c|c|c|c|c|c|c|c|c|}
\hline Type & 1 & II & III & IV & V & $\mathrm{VI}$ & VII & VIII & IX & $X$ & XI & XII & XII & XIV & $\mathrm{XV}$ & $\mathrm{XVI}$ & XVII \\
\hline Min. & 838.76 & 801.01 & 789.42 & 789.72 & 845.90 & 831.88 & 816.71 & 800.22 & 788.14 & 845.86 & 833.03 & 819.05 & 786.62 & 829.96 & 792.87 & 783.84 & 790.24 \\
\hline Max. & 1777.81 & 1740.06 & 1728.47 & 1728.76 & 1784.95 & 1770.93 & 1755.75 & 1739.26 & 1727.18 & 1784.90 & 1772.07 & 1758.09 & 1725.66 & 1769.00 & 1731.91 & 1722.88 & 1729.29 \\
\hline
\end{tabular}

\section{5\% Premium}

\begin{tabular}{|c|c|c|c|c|c|c|c|c|c|c|c|c|c|c|c|c|c|}
\hline Type & 1 & II & III & IV & V & $\mathrm{VI}$ & VII & VIII & IX & $X$ & $\mathrm{XI}$ & XII & XII & XIV & $X V$ & $\mathrm{XVI}$ & XVII \\
\hline Mean & 1185.26 & 1147.51 & 1135.92 & 1136.22 & 1192.40 & 1178.38 & 1163.21 & 1146.72 & 1134.64 & 1192.36 & 1179.53 & 1165.55 & 1133.12 & 1176.46 & 1139.37 & 1130.34 & 1136.74 \\
\hline Std.Dev & 149.43 & 149.43 & 149.43 & 149.43 & 149.43 & 149.43 & 149.43 & 149.43 & 149.43 & 149.43 & 149.43 & 149.43 & 149.43 & 149.43 & 149.43 & 149.43 & 149.43 \\
\hline Min. & 799.25 & 761.50 & 749.91 & 750.21 & 806.39 & 792.37 & 777.20 & 760.70 & 748.62 & 806.35 & 793.51 & 779.54 & 747.10 & 790.45 & 753.36 & 744.33 & 750.73 \\
\hline Max. & 1691.34 & 1653.59 & 1642.00 & 1642.30 & 1698.48 & 1684.46 & 1669.29 & 1652.80 & 1640.72 & 1698.44 & 1685.61 & 1671.63 & 1639.20 & 1682.54 & 1645.45 & 1636.42 & 1642.82 \\
\hline
\end{tabular}

\section{0\% Premium}

\begin{tabular}{|c|c|c|c|c|c|c|c|c|c|c|c|c|c|c|c|c|c|}
\hline Type & 1 & II & III & TV & V & $\mathrm{VI}$ & VII & VIII & IX & $\mathrm{X}$ & $\mathrm{XI}$ & $\mathrm{XII}$ & $\mathrm{XII}$ & XIV & $X V$ & $\mathrm{XVI}$ & $\mathrm{XVII}$ \\
\hline Mean & 1128.42 & 1090.68 & 1079.09 & 1079.38 & 1135.57 & 1121.54 & 1106.37 & 1089.88 & 1077.80 & 1135.52 & 1122.69 & 1108.71 & 1076.28 & 1119.62 & 1082.53 & 1073.50 & 1079.90 \\
\hline Std.Dev & 141.96 & 141.96 & 141.96 & 141.96 & 141.96 & 141.96 & 141.96 & 141.96 & 141.96 & 141.96 & 141.96 & 141.96 & 141.96 & 141.96 & 141.96 & 141.96 & 141.96 \\
\hline Min. & 761.71 & 723.97 & 712.38 & 712.67 & 768.86 & 754.83 & 739.66 & 723.17 & 711.09 & 768.81 & 755.98 & 742.00 & 709.57 & 752.91 & 715.82 & 706.79 & 713.19 \\
\hline Max. & 1609.20 & 1571.45 & 1559.86 & 1560.16 & 1616.34 & 1602.32 & 1587.15 & 1570.65 & 1558.57 & 1616.30 & 1603.47 & 1589.49 & 1557.05 & 1600.40 & 1563.31 & 1554.28 & 1560.68 \\
\hline
\end{tabular}


Case B: Descriptive Statistics of Net Distribution Returns (\$) (Actual End Value: \$89.79).

Table 1-8.: Descriptive Statistics of Net Return Distributions of 17 different insurances choices (Actual End Value: \$89.79).

Base Price Scenario:

\begin{tabular}{|c|c|c|c|c|c|c|c|c|c|c|c|c|c|c|c|c|c|}
\hline Type & $\mathrm{T}$ & III & III & IV & $\mathrm{V}$ & VI & VII & VIII & IX & $\mathrm{X}$ & $\mathrm{XI}$ & XII & XII & XIV & $\mathrm{XV}$ & XVI & XVII \\
\hline Mean & 1401.54 & 1393.19 & 1394.80 & 1395.10 & 1408.68 & 1394.66 & 1384.59 & 1388.09 & 1393.51 & 1408.64 & 1395.81 & 1381.83 & 1387.69 & 1392.74 & 1377.65 & 1389.22 & 1395.62 \\
\hline Std.Dev & 183.46 & 183.46 & 183.46 & 183.46 & 183.46 & 183.46 & 183.46 & 183.46 & 183.46 & 183.46 & 183.46 & 183.46 & 183.46 & 183.46 & 183.46 & 183.46 & 183.46 \\
\hline Min. & 927.62 & 919.27 & 920.88 & 921.18 & 934.76 & 920.74 & 910.67 & 914.17 & 919.59 & 934.72 & 921.88 & 907.90 & 913.77 & 918.82 & 903.73 & 915.30 & 921.70 \\
\hline Max. & 2022.87 & 2014.53 & 2016.14 & 2016.43 & 2030.02 & 2015.99 & 2005.92 & 2009.43 & 2014.85 & 2029.97 & 2017.14 & 2003.16 & 2009.03 & 2014.07 & 1998.98 & 2010.55 & 2016.95 \\
\hline
\end{tabular}

Discounted Price Scenario:

\begin{tabular}{|c|c|c|c|c|c|c|c|c|c|c|c|c|c|c|c|c|c|}
\hline Type & $T$ & III & III & IV & V & VI & VII & VIII & IX & $X$ & $\mathrm{XI}$ & XII & $\mathrm{XII}$ & XIV & $X V$ & $\mathrm{XVI}$ & XVII \\
\hline Mean & 1331.17 & 1322.82 & 1324.43 & 1324.73 & 1338.31 & 1324.29 & 1314.22 & 1317.72 & 1323.14 & 1338.26 & 1325.43 & 1311.45 & 1317.32 & 1322.37 & 1307.27 & 1318.85 & 1325.84 \\
\hline Std.Dev & 173.37 & 173.37 & 173.37 & 173.37 & 173.37 & 173.37 & 173.37 & 173.37 & 173.37 & 173.37 & 173.37 & 173.37 & 173.37 & 173.37 & 173.37 & 173.37 & 174.28 \\
\hline Min. & 881.53 & 873.19 & 874.80 & 875.09 & 888.68 & 874.65 & 864.58 & 868.09 & 873.51 & 888.63 & 875.80 & 861.82 & 867.69 & 872.73 & 857.64 & 869.21 & 875.61 \\
\hline Max. & 1918.00 & 1909.65 & 1911.26 & 1911.56 & 1925.14 & 1911.12 & 1901.05 & 1904.55 & 1909.97 & 1925.09 & 1912.26 & 1898.28 & 1904.15 & 1909.20 & 1894.10 & 1905.67 & 1916.11 \\
\hline
\end{tabular}




\section{5\% Premium}

\begin{tabular}{|c|c|c|c|c|c|c|c|c|c|c|c|c|c|c|c|c|c|}
\hline Type & 1 & II & III & IV & V & VI & VII & VIII & IX & $X$ & $\mathrm{XI}$ & XII & XII & XIV & $X V$ & XVI & XVII \\
\hline Mean & 1264.91 & 1256.56 & 1258.17 & 1258.46 & 1272.05 & 1258.03 & 1247.95 & 1251.46 & 1256.88 & 1272.00 & 1259.17 & 1245.19 & 1251.06 & 1256.10 & 1241.01 & 1252.58 & 1259.55 \\
\hline Std.Dev & 164.70 & 164.70 & 164.70 & 164.70 & 164.70 & 164.70 & 164.70 & 164.70 & 164.70 & 164.70 & 164.70 & 164.70 & 164.70 & 164.70 & 164.70 & 164.70 & 165.57 \\
\hline Min. & 837.75 & 829.41 & 831.02 & 831.31 & 844.90 & 830.87 & 820.80 & 824.31 & 829.73 & 844.85 & 832.02 & 818.04 & 823.91 & 828.95 & 813.86 & 825.43 & 831.83 \\
\hline Max. & 1822.39 & 1814.05 & 1815.66 & 1815.95 & 1829.54 & 1815.51 & 1805.44 & 1808.95 & 1814.37 & 1829.49 & 1816.66 & 1802.68 & 1808.55 & 1813.59 & 1798.50 & 1810.07 & 1820.30 \\
\hline
\end{tabular}

\section{0\% Premium}

\begin{tabular}{|c|c|c|c|c|c|c|c|c|c|c|c|c|c|c|c|c|c|}
\hline Type & 1 & III & III & IV & V & $\mathrm{VI}$ & VII & VIII & IX & $\mathrm{X}$ & $\mathrm{XI}$ & XII & XII & XIV & $X V$ & XVI & XVII \\
\hline Mean & 1201.96 & 1193.61 & 1195.22 & 1195.51 & 1209.10 & 1195.08 & 1185.01 & 1188.51 & 1193.93 & 1209.05 & 1196.22 & 1182.24 & 1188.11 & 1193.16 & 1178.06 & 1189.63 & 1196.57 \\
\hline Std.Dev & 156.46 & 156.46 & 156.46 & 156.46 & 156.46 & 156.46 & 156.46 & 156.46 & 156.46 & 156.46 & 156.46 & 156.46 & 156.46 & 156.46 & 156.46 & 156.46 & 157.29 \\
\hline Min. & 796.16 & 787.81 & 789.42 & 789.72 & 803.30 & 789.28 & 779.21 & 782.72 & 788.14 & 803.26 & 790.43 & 776.45 & 782.32 & 787.36 & 772.27 & 783.84 & 790.24 \\
\hline Max. & 1731.57 & 1723.22 & 1724.83 & 1725.13 & 1738.71 & 1724.69 & 1714.62 & 1718.12 & 1723.54 & 1738.67 & 1725.84 & 1711.86 & 1717.73 & 1722.77 & 1707.68 & 1719.25 & 1729.29 \\
\hline
\end{tabular}

\section{5\% Premium}

\begin{tabular}{|c|c|c|c|c|c|c|c|c|c|c|c|c|c|c|c|c|c|}
\hline Type & $T$ & III & TII & TV & $\nabla$ & VI & VII & VIII & TX & $X$ & $\mathrm{XI}$ & XII & XII & XIV & $X V$ & $X \nabla \Gamma$ & $X \nabla I I$ \\
\hline Mean & 1142.15 & 1133.81 & 1135.42 & 1135.71 & 1149.30 & 1135.27 & 1125.20 & 1128.71 & 1134.13 & 1149.25 & 1136.42 & 1122.44 & 1128.31 & 1133.35 & 1118.26 & 1129.83 & 1136.74 \\
\hline Std.Dev & 148.64 & 148.64 & 148.64 & 148.64 & 148.64 & 148.64 & 148.64 & 148.64 & 148.64 & 148.64 & 148.64 & 148.64 & 148.64 & 148.64 & 148.64 & 148.64 & 149.43 \\
\hline Min. & 756.65 & 748.30 & 749.91 & 750.21 & 763.79 & 749.77 & 739.70 & 743.20 & 748.62 & 763.75 & 750.91 & 736.94 & 742.80 & 747.85 & 732.76 & 744.33 & 750.73 \\
\hline
\end{tabular}




\begin{tabular}{|c|c|c|c|c|c|c|c|c|c|c|c|c|c|c|c|c|c|}
\hline Type & $T$ & II & III & TV & V & VI & VII & VIII & TX & $X$ & $\mathrm{XI}$ & XII & XII & XIV & $X V$ & $\mathrm{XVI}$ & XVII \\
\hline Max. & 1645.29 & 1636.94 & 1638.55 & 1638.85 & 1652.43 & 1638.41 & 1628.34 & 1631.84 & 1637.26 & 1652.38 & 1639.55 & 1625.57 & 1631.44 & 1636.49 & 1621.39 & 1632.96 & 1642.82 \\
\hline
\end{tabular}

20\% Premium

\begin{tabular}{|c|c|c|c|c|c|c|c|c|c|c|c|c|c|c|c|c|c|}
\hline Type & 1 & II & III & IV & V & $\mathrm{VI}$ & VII & VIII & IX & $X$ & $\mathrm{XI}$ & XII & XII & XIV & $X V$ & $X V I$ & XVII \\
\hline Mean & 1085.34 & 1077.00 & 1078.61 & 1078.90 & 1092.49 & 1078.46 & 1068.39 & 1071.90 & 1077.32 & 1092.44 & 1079.61 & 1065.63 & 1071.50 & 1076.54 & 1061.45 & 1073.02 & 1079.90 \\
\hline Std.Dev & 141.21 & 141.21 & 141.21 & 141.21 & 141.21 & 141.21 & 141.21 & 141.21 & 141.21 & 141.21 & 141.21 & 141.21 & 141.21 & 141.21 & 141.21 & 141.21 & 141.96 \\
\hline Min. & 719.11 & 710.77 & 712.38 & 712.67 & 726.26 & 712.23 & 702.16 & 705.67 & 711.09 & 726.21 & 713.38 & 699.40 & 705.27 & 710.31 & 695.22 & 706.79 & 713.19 \\
\hline Max. & 1563.32 & 1554.97 & 1556.58 & 1556.88 & 1570.46 & 1556.44 & 1546.37 & 1549.87 & 1555.29 & 1570.42 & 1557.58 & 1543.61 & 1549.47 & 1554.52 & 1539.43 & 1551.00 & 1560.68 \\
\hline
\end{tabular}

In case A, where the actual LRP end values were used, no indemnity was paid in any price scenario. So, in our stochastic dominance analysis in Simetar, no insurance (Choice XVII) was picked as the most preferred option. The net return distributions were used in

Case B to analyze the stochastic dominance. Results are reported in the following graphs.




\section{A. CDFs of Case B:}
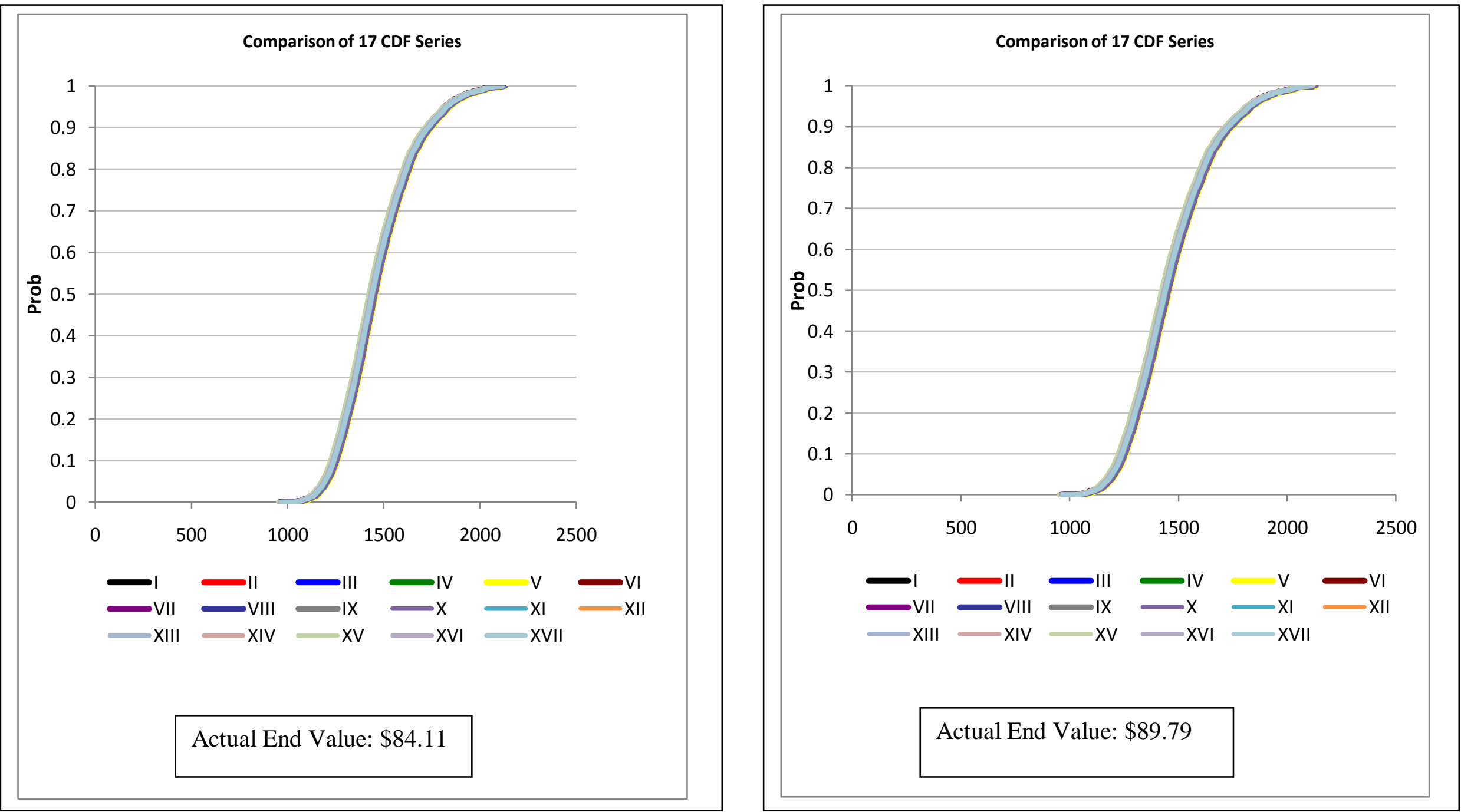

Figure 1-10.: CDFs of 17 different choices of various price scenarios of Case B. 


\section{B. Risk Rankings Using FSD}

Table 1-9.: Risk Ranking of Case B with Actual End Value of $\$ 84.11$

\begin{tabular}{|c|c|c|}
\hline & \multicolumn{2}{|c|}{ Actual End Value: \$84.11 } \\
\hline Risk Rank & Insurance Choice Level of Preference as determined by FSD \\
\hline 1 & V & Most Preferred \\
\hline 2 & X & 2nd Most Preferred \\
\hline 3 & I & 3rd Most Preferred \\
\hline 4 & XI & 4th Most Preferred \\
\hline 5 & VI & 5th Most Preferred \\
\hline 6 & XIV & 6th Most Preferred \\
\hline 7 & XVII & 7th Most Preferred \\
\hline 8 & IV & 8th Most Preferred \\
\hline 9 & III & 9th Most Preferred \\
\hline 10 & IX & 10th Most Preferred \\
\hline 11 & II & 11th Most Preferred \\
\hline 12 & XVI & 12th Most Preferred \\
\hline 13 & VIII & 13th Most Preferred \\
\hline 14 & XIII & 14th Most Preferred \\
\hline 15 & XII & 15th Most Preferred \\
\hline 16 & VII & 16th Most Preferred \\
\hline 17 & XV & Least Preferred \\
\hline
\end{tabular}


Table 1-10.: Risk Ranking of Case B with Actual End Value of \$89.79

\begin{tabular}{|c|c|c|}
\hline & \multicolumn{2}{|c|}{ Actual End Value: \$89.79 } \\
\hline $\begin{array}{c}\text { Risk } \\
\text { Rank }\end{array}$ & Name & $\begin{array}{c}\text { Level of Preference as } \\
\text { determined by FSD }\end{array}$ \\
\hline 1 & VI & Most Preferred \\
\hline 2 & XI & 2nd Most Preferred \\
\hline 3 & I & 3rd Most Preferred \\
\hline 4 & X & 4th Most Preferred \\
\hline 5 & V & 5th Most Preferred \\
\hline 6 & XIV & 6th Most Preferred \\
\hline 7 & XVII & 7th Most Preferred \\
\hline 8 & IV & 8th Most Preferred \\
\hline 9 & III & 9th Most Preferred \\
\hline 10 & IX & 10th Most Preferred \\
\hline 11 & II & 11th Most Preferred \\
\hline 12 & XVI & 12th Most Preferred \\
\hline 13 & VIII & 13th Most Preferred \\
\hline 14 & XIII & 14th Most Preferred \\
\hline 15 & XII & 15th Most Preferred \\
\hline 16 & VII & 16th Most Preferred \\
\hline 17 & XV & Least Preferred \\
\hline
\end{tabular}


Analyzing the Cumulative Distribution Functions (collectively CDFs) and the risk rankings as determined using Simetar, it can be seen that individual rankings and CDFs for different price scenarios remain unchanged. There is little difference in the individual rankings of two LRP end values. Choice V and VI have been selected as the most preferred insurance option for the producers for end values of \$84.xx and \$89.xx, respectively. They correspond to 17 weeks coverage, with a coverage price of $\$ 93.66$ and $\$ 91.66$, respectively, for choices $\mathrm{V}$ and VI. A recommendation to these producers would be to go for the 17 weeks coverage option. However, due to the basis that exists between the local cattle markets vs. AMS 5 area market; this insurance policy may not be useful to WV producers, due to the fact that an indemnity will not be paid out. The basis is reported in figure 1.3. It is obvious from 5 years of historical data that the local cattle auction markets operate at a much lower price as compared to the AMS 5 area market at least for the heavy steer/heifer $(500+1 b s)$ category. Since the indemnity payout is typically tied to the AMS price, these producers may not be able to receive any indemnity at all.

\subsection{Black's (1976) Model to Price the LRP Premium}

After starting the initial work in April 2009, it was difficult to understand how USDA/RMA calculates the premium and coverage levels of LRP. Subsequently, after contact, USDA/RMA declined to give any insight as to how LRP premiums are priced. However, the reported LRP premium prices can be evaluated using Black's (1976) model, which is actually an approximation of the Black-Scholes model for option pricing. Here the stock price needs to be replaced by the futures price. The Black model can be written as [Hull, 2007]: 
Price of a put option on futures is given by:

$$
\begin{aligned}
& \mathrm{p}=\mathrm{e}^{-\mathrm{rT}}\left[\mathrm{KN}\left(-\mathrm{d}_{2}\right)-\mathrm{FN}\left(-\mathrm{d}_{1}\right)\right] \\
& \mathrm{d}_{1}=\frac{\left[\ln \left(\frac{\mathrm{F}}{\mathrm{K}}\right)+\sigma^{2} \frac{\mathrm{T}}{2}\right]}{\sigma \sqrt{\mathrm{T}}} ; \\
& \mathrm{d}_{2}=\frac{\left[\ln \left(\frac{\mathrm{F}}{\mathrm{K}}\right)-\sigma^{2} \frac{\mathrm{T}}{2}\right]}{\sigma \sqrt{\mathrm{T}}}
\end{aligned}
$$

Where, $\mathrm{r}$ is the risk-free interest rate, $\mathrm{T}$ is the time to maturity; $\mathrm{K}$ is the strike/exercise rate, $\mathrm{F}$ is the futures (or forward) price, $\sigma$ is the unknown price volatility and $\mathrm{N}(\mathrm{x})$ is the cumulative probability or area under the normal curve.

The derivation of the pricing formulas in the model follows that of the Black-Scholes model almost exactly. The assumption that the spot price follows a log-normal process (in Black-Scholes model) is replaced by the assumption that the forward price at maturity of the option is log-normally distributed (in Black Model). From there, the derivation is identical, and so the final formula is the same except that the spot price is replaced by the forward - the forward price represents the undiscounted expected future value.

An example of using the Black-Model: The following example will make the above discussion clear. Take us for an example of a Put option which had been traded over CME on Oct. 15, 2008, with a delivery date of 24th March, 2009. The features of this put contract is the Future price $(\mathrm{F})$ is $\$ 104.08$. Strike rate is $\$ 88$. Price of this put option $\mathrm{p}$ is $\$ 0.75 / \mathrm{cwt}$ before brokerage commission. A Similar LRP with end date March 11, 2009 cost is $\$ 0.78 / \mathrm{cwt}$ before subsidy. USDA/RMA provides $13 \%$ subsidy to LRP coverage. So the effective price after subsidy is $\$ 0.68 / \mathrm{cwt}$. Black Model can be used successfully to show that these two hedging mechanism (put and LRP) are actually cost almost the same. I used the Black Model 
to calculate volatility from traded put and when put terms adjusted according to LRP, put price $=\$ 0.64+\$ 0.12$ (broker commission) $=\$ 0.76$. Put terms (length) needs to be adjusted to reflect the similar LRP features.

LRP and put options are similar, but not identical. LRP premiums receive a federal price subsidy. A brokerage commission adds to the quoted cost of a put option. LRP contracts are written on underlying forward prices, called the "expected ending value" in the policy endorsement. For feeder cattle and swine LRP endorsements, the forward and futures prices settle to the same cash prices. For the fed cattle LRP endorsement, the forward prices are similar to the cash price used to settle live cattle futures contracts. LRP coverage is available daily with ending dates a fixed number of weeks in the future. Put options have set expiration dates and set strike prices. LRP coverage is available at fixed percentages of the expected ending value, in essence becoming strike prices.

\subsection{Conclusion}

LRP offers livestock producers the opportunity to manage a portion of their price risk by buying insurance coverage for cattle. The decision to manage price risk is very attractive in today's environment where grain and input prices, international trade, and the U.S. economy have added to the volatility and uncertainty in feeder/fed cattle prices. However, LRP's usefulness remains doubtful particularly to grass fed beef producers in West Virginia. This insurance program may work well to reduce the market risk of feeder cattle, but is not very effective for the heavy cattle (i.e. fed cattle) category. This program adds one more option in the arsenal of risk management strategies of grass fed producers who have historically purchased CME traded futures and puts on those futures. Future research needs to be conducted on the stochastic dominance analysis of the equivalent LRP and CME traded put options to determine which strategy works better for these producers. Either of them have 
their unique advantages and disadvantages. So, advanced risk managers need to identify which one will work better for them. But it is to be kept in mind that none of these risk management strategies absolutely guarantees a profit over production cost as both of them are tied to market movement. 


\section{LIST OF REFERENCES}

\section{A. Journal Articles}

Antle, J.1987. Econometric estimation of producer's risk attitudes. American Journal of Agricultural Economics 69: 509-522.

Atwood, J.A., J.F. Robinson-Cox, and S. Shaik. 2006. Estimating the Prevalence and Cost of Yield Switching Fraud in the Federal Crop Insurance Program. American Journal of Agricultural Economics 88(I-2):365-381.

Babcock, B.A., and D. Hennessy. 1996. Input Demand under Yield and Revenue Insurance. American Journal of Agricultural Economics 78:416-427.

Chambers, R.G. Insurability and Moral Hazard in Agricultural Insurance Markets.1989. American Journal of Agricultural Economics 71 (August 1989):604-16.

Chandler, R., and H. Wheater. 2002. Analysis of rainfall variability using generalized linear models: a case study from the west of Ireland. Water Resources Research 38: 1192-1202.

Chavas, J.P., Holt, M.T. 1996. Economic behavior under uncertainty: a joint analysis of risk preferences and technology. Review of Economics and Statistics 78: 329-335.

Evans, J., M. Sperow, G. D’Souza, and E.B. Rayburn. 2007. Stochastic simulation of pasture raised beef production systems and implications for the Appalachian cow-calf sector. Journal of Sustainable Agriculture 30(4): 27-51.

Follmer, H., and P. Leukert. 1999. Quantile Hedging. Finance and Stochastics 3: 251-73.

Follmer, H., and P. Leukert. 2000. Efficient Hedging: Cost versus Shortfall Risk. Finance and Stochastics 4: 117-46.

Follmer, H., and D. Sondermann. 1986. In: W. Hildenbrand and A. Mas-Colell, eds., Hedging of Non-redundant Contingent Claims, Contributions to Mathematical Economics (Amsterdam: North-Holland).

Glauber, J.W., and K.J. Collins. Crop Insurance, Disaster Assistance, and the Role of the Federal Government in Providing Catastrophic Risk Protection. Agricultural Finance. Rev. 62:2(Fall 2002):81-102.

Hallman, A., Lowenberg-DeBoer, J.M. 1999. Cost, average returns and risk of switching to narrow row corn. Journal of Crop Production 12:685-691.

Hardarker, J.B., J.W. Richardson, G. Lien, and K.D. Schumann. 2004. Stochastic Efficiency Analysis with Risk Aversion Bounds: A Simplified Approach. Australian Journal of Agricultural and Resource Economics 48(I-2):253-270. 
Hien, V., Kabore, D., Youl, S., and Lowenberg-DeBoer, J., 1997. Stochastic dominance analysis of on-farm-trial data: The riskiness of alternative phosphate sources in Burkina Faso. Agricultural Economics 15 (I-3):213-221.

Jewson, S., A. Brix, and C. Ziehmann. 2002. Getting the Price Right. Global Reinsurance February: 10-15.

Just, R.E., L. Calvin, and J. Quiggen. 1999. Adverse Selection in Crop Insurance: Actuarial and Asymmetric Information Incentives. American Journal of Agricultural Economics 81:834-849.

J.W. Pratt. 1964. Risk Aversion in the Small and in the Large. Econometrica 32 (I-1): 122136.

Kaylen, M.S., E.T. Loehman, and P.V. Preckel. 1989. Farm-level Analysis of Agricultural Insurance: A Mathematical Programming Approach. Agricultural Systems, 30:235-244.

Kroll, Y., H. Levy, and H. M. Markowitz. 1984. Mean-Variance versus Direct Utility Maximization." Journal of Finance 39: 47-61.

Lambert, D.K., and B.A. McCarl. 1985. Risk-Modeling Using Direct Solution of Nonlinear Approximations of the Utility Function. American Journal of Agricultural Economics $67: 846-852$.

Lintner, John. 1965. The Valuation of Risk Assets and the Selection of Risky Investments in Stock Portfolios and Capital Budgets. Review of Economics and Statistics 47(I-1):13-37.

May, K.O.1954. Intransitive, utility and the aggregation of preference patterns, Econometrica 22:1-13.

McCarl, B.A., and D.A. Bessler. 1989. Estimating an Upper Bound on the Pratt Risk Aversion Coefficient When the Utility Function is Unknown. Australian Journal of Agricultural and Resource Economics 33(I-1):55-63.

MacDonald, J., J. Perry, M. Ahearn, D. Banker, W. Chambers, C. Dmimtri, N. Key, K. Nelson and L. Southard. Contracts, Markets and Prices. 2004. In USDA, Economic Research Service, Ag Economic Report No. 837, November 2004.

Meyer, Jack. 1977. Choice among Distributions. Journal of Economic Theory 14: 326-36.

Meyer, Jack. 1977. Second Degree Stochastic Dominance with Respect to a Function. International Economic Review 18: 477-487.

Moss, C.B. 2005. Lecture on stochastic dominance. Class notes, Agricultural Decision Making under Risk and Uncertainty (AEB 6582). University of Florida, Gainesville. 
Racine, J., and A. Ker. 2006. Rating Crop Insurance Policies with Efficient Nonparametric Estimators that Admit Mixed Data Types. Journal of Agricultural and Resource Economics 31 (I-1): 27-39.

Rejesus, R.M., K.H. Coble, T.O. Knight, and Y. Jin. 2006. Developing Experience-Based Premium Rate Discounts in Crop Insurance. American Journal of Agricultural Economics 88(I-2):409-419.

Ribera, L.A., F.M. Hons, and J.W. Richardson. 2004. Tillage and Cropping Systems: An Economic Comparison between Conventional and No-Tillage Farming Systems in Burleson County, Texas. Agronomy Journal 96:415-424.

Sangtaek, S., P.D. Mitchell, and D.J. Leatham. 2005. Effects of Federal Risk Management Programs on Optimal Acreage Allocation and Nitrogen Use in a Texas Cotton-Sorghum System. Journal of Agricultural and Applied Economics 37(I-3): 26-30.

Spiselman, A. 2006. Is grass-fed greener? Meatingplace, "In the Aisles" section, September issue: $74-77$.

Tversky, A. 1969. Intransitivity of preferences. Psychological Review 76: 31-48.

\section{B. Books}

Anderson, Dillon and Hardeker, 1977. Agricultural Decision Analysis. Iowa State University Press, Ames,Iowa.

Borch, Karl Henrik. 1968. The Economics of Uncertainty. Princeton University Press, Princeton, New Jersey. Chapters 2-4.

Hey, John D. , 1979, Uncertainty in Microeconomics. New York University Press, New York, New York.

Hull, John, 2008. Options, Futures and Other Derivatives. Sixth Edition. Prentice Hall of India Pvt. Ltd, New Delhi, India.

Tisdell, Clement A, 1968, The Theory of Price Uncertainty, Production, and Profit. Princeton University Press, Princeton, New Jersey.

Varian, H.R. 1991. Microeconomic Analysis, Third Edition. W.W. Norton \& Co, New York ,New York.

\section{Websites}

LRP premium calculator from USDA-RMA; 
Available at: http://www3.rma.usda.gov/apps/livestock_reports/main.aspx.

Online Livestock Trading Information at CME; Available at: cmegroup.com/trading/commodities/.

USDA/National Agricultural Statistics Service (NASS). 2007. Selected data. Available web at www.nass.usda.gov.

USDA/RMA web site; available at: http://www.rma.usda.gov/. 


\title{
CHAPTER 2
}

\section{PRODUCTION RISK AND RAINFALL INSURANCE FROM USDA/RMA: A} STOCHASTIC DOMINANCE ANALYSIS

\author{
Remember, when discoursing about water, \\ to induce first experience, then reason. \\ - Leonardo Da Vinci
}

\section{1. Introduction}

Crop insurance is purchased by agricultural producers, including farmers, ranchers, and others to protect themselves against the loss of their crops due to natural disasters. These natural disasters include hail, drought, and even floods. The two general categories of crop insurance are: crop-yield insurance and crop-revenue insurance.

\subsubsection{Crop-Yield Insurance}

There are two main classes of crop-yield insurance: the first class is hail insurance, which is generally available from private insurers because hail damage is limited in the manner by which it occurs, and it is localized. The accumulated losses accrued from hail damage being small in compensatory amount tend not to overwhelm the capital reserves of private insurers. The earliest crop-hail programs were the result of farmers' cooperatives in France and Germany in the 1820s (Fact sheet, European Commission on Agriculture, 2009).

Multi-peril crop insurance covers the broad perils of drought, flood, insects, disease, etc., which may affect farmers insured simultaneously. This crop insurance protects the insurer with excessive losses. To make this class of insurance, the perils are often bundled together in a single policy, called a multi-peril crop insurance (MPCI) policy. MPCI coverage is normally offered by a government insurer and premiums are usually partially subsidized by the government. The earliest MPCI program was implemented by the Federal 
Crop Insurance Corporation (FCIC), an agency of the U.S. Department of Agriculture (USDA), in 1938. The FCIC program has been managed by the Risk Management Agency (RMA) since 1996.

\subsubsection{Crop-Revenue Insurance:}

This insurance is a combination of both crop-yield insurance and price insurance. RMA establishes crop-revenue insurance guarantees on corn by multiplying each farmer's cornyield guarantee, which is based on the farmer's own production history, times the harvesttime futures price discovered at a commodity exchange before the policy is sold and the crop planted. There is a single guarantee for a certain number of dollars. The policy pays an indemnity if the combination of the actual yield and the cash settlement price in the futures market (as determined by trading at CME) is less than the guarantee. In the United States, the program is called Crop Revenue Coverage according to information available from USDA/RMA. Crop-revenue insurance covers the decline in price that occurs during that specific insured growing season. The insurance does not cover declines that may occur from one growing season to another.

\subsection{Insurance for Pasture/Rangeland Management: Rainfall/Vegetation Index Based Insurance}

The United States currently comprises about 588 million acres of pasture and rangeland and 61.5 million acres of hay land. RMA has developed programs for pasture, rangeland, forage, and hay to provide a safety net for farmers who face drought conditions (USDA/RMA, 2008). This safety net is currently comprised of two programs: the Rainfall Index program

and the Vegetation Index program. Both programs use indexes and grids that are smaller in 
size than counties to determine expected losses. The Rainfall program is based on accumulated rainfall and the Vegetation program relies on satellite imagery to measure departures from expected losses for a given grid area. As of January 2009, the Rainfall Index is available for at least 10 states and the vegetation index for at least 13 states. So far, they have been highly successful due to the participation levels occurring in excess of expectations. Together, these programs ultimately will be available for areas that represent about 25 percent of the nation's grazing and hay land. The RMA's long-range goals call for crop insurance to be available that covers 98 percent of the value of U.S. commercial crops by crop year 2012 .
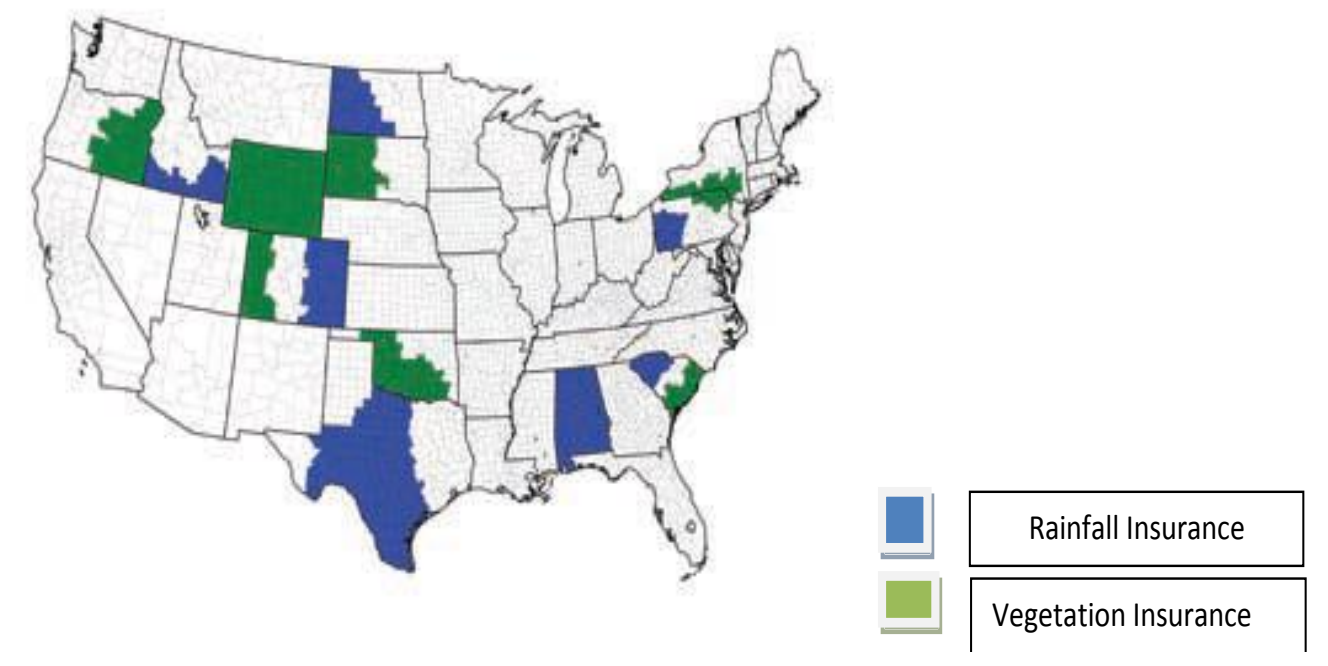

Figure 2-1.: Pasture, Rangeland and Forage Program 2008 Crop Year.

\subsection{Rainfall Insurance}

In this section, various elements of the rainfall insurance, which has been marketed by RMA since 2009, will be discussed. These definitions were collected from policy and actuarial documents maintained at RMA website. 
1. Grid - Acreage within each 0.25 degree gridded area established by National Oceanic and Atmospheric Administration (NOAA) and identified by longitude and latitude.

The grid is used to determine the expected grid index, premium, final grid index, and indemnity.

2. Grid identification number (grid ID) - A specific number code associated with each grid contained in the actuarial documents. The applicable grid ID is determined based on a point of reference selected by the farmer, which must be established by the acreage reporting date.

3. Index interval - A period of time during which precipitation data is collected that is used to calculate the expected grid index and final grid index, which is designated on the Special Provisions.

4. Insurable acres - The total number of acres planted or established, as applicable to the insured crop and eligible for insurance under the applicable Crop Provisions. Insurable acres include both insured acres and acres of the crop that are not insured (if allowed by the Crop Provisions) in the county.

5. Coverage Level - The coverage level is the percentage of the county base value selected by a producer for insurance coverage. A producer can chose a coverage level of $70,75,80,85$, or 90 percent of the county base value for the crop type being insured. Producers are required to insure all grids in a county at the same coverage level.

6. Productivity Factor - The productivity factor is a factor between 60 and 150 percent that a producer selects to reflect his/her operation's forage productivity. Producers often select coverage levels and productivity factors to reflect the forage production 
value on the acreage they are insuring. For instance, if the producer realizes that his/her value of production is similar to the county base value, the producer may select a coverage value/productivity factor combination to provide a level of protection similar to the county base value for the crop type that is being insured.

7. Producer Share - The producer share is the operator's share of forage production on the insured acreage. If a producer is an owner/operator, his/her share is likely to be 100 percent. But if a producer hays acreage on a 50/50 crop share basis, his/her share is 50 percent.

8. Premium Calculations: Premium calculations for a Pasture, Rangeland and Forage PRF Rainfall Index Insurance contract are similar to those of other group risk insurance products. The premium rate is quoted as a dollar amount per $\$ 100$ of insurance liability (the maximum indemnity payment under the provisions of the contract which equals the dollar protection per acre chosen by the producer). The premium rate is multiplied by an adjustment factor of 0.01 to express the premium rate on a per dollar of protection basis. Premium subsidy rates are similar to those for other group risk products and subsidy rates decrease as coverage levels increase.

9. PRF Rainfall Index Insurance Indemnities: Indemnities are paid to a producer when the rainfall final grid index falls below the trigger grid index established by the producer.

Trigger Grid Index $=($ Expected Grid Index $) \mathrm{x}($ Coverage Level $)(11)$ 
If an insured producer wants a relatively high Trigger Grid Index, they will choose a relatively high coverage level. The amount of the indemnity is determined by a payment calculation factor, defined as $\frac{\text { (Trigger Grid Index -Final Grid Index) }}{\text { (Trigger Grid Index })}$ (12).

An insurance payment (indemnity) is due if the Final Grid Index is less than the Trigger Grid Index. The indemnity per unit is: Policy Protection per Unit x Payment Calculation Factor. The Final Grid Index values are calculated soon after the close of each index interval so that insurance payments can be made in time.

The website maintained by RMA has a decision support tool (Appendix B) which gives the entire premium and indemnity related information and the data were collected from this decision tool only.

\subsection{Empirical Model}

An empirical model has been developed to measure risk in grass fed beef production and to evaluate how these producers would respond to the livestock risk protection plan of USDA/RMA. The distribution of payoffs from this model was evaluated using First Degree Dominance or FSD, similar to the previous essay. The analysis again builds upon other recent studies using stochastic dominance to evaluate risks and cropping decisions as have been discussed in the previous essay. The framework underlying the economics of insurance is outlined in Dionne and Harrington (1992) and Jewson and Brix (2005).

The net return is given by:

Net Return $=$ Net Revenue + Indemnity Paid Out - Premium Paid + Production Cost (13)

Production cost is not included as we assume that it is fixed or constant for all the cases. Hence, our model reduces to:

Net Return $=$ Net Revenue + Indemnity Paid Out - Premium Paid $\quad$ (14) 
Methodology Flowchart to Carry Out Stochastic Dominance Analysis of Rainfall Insurance

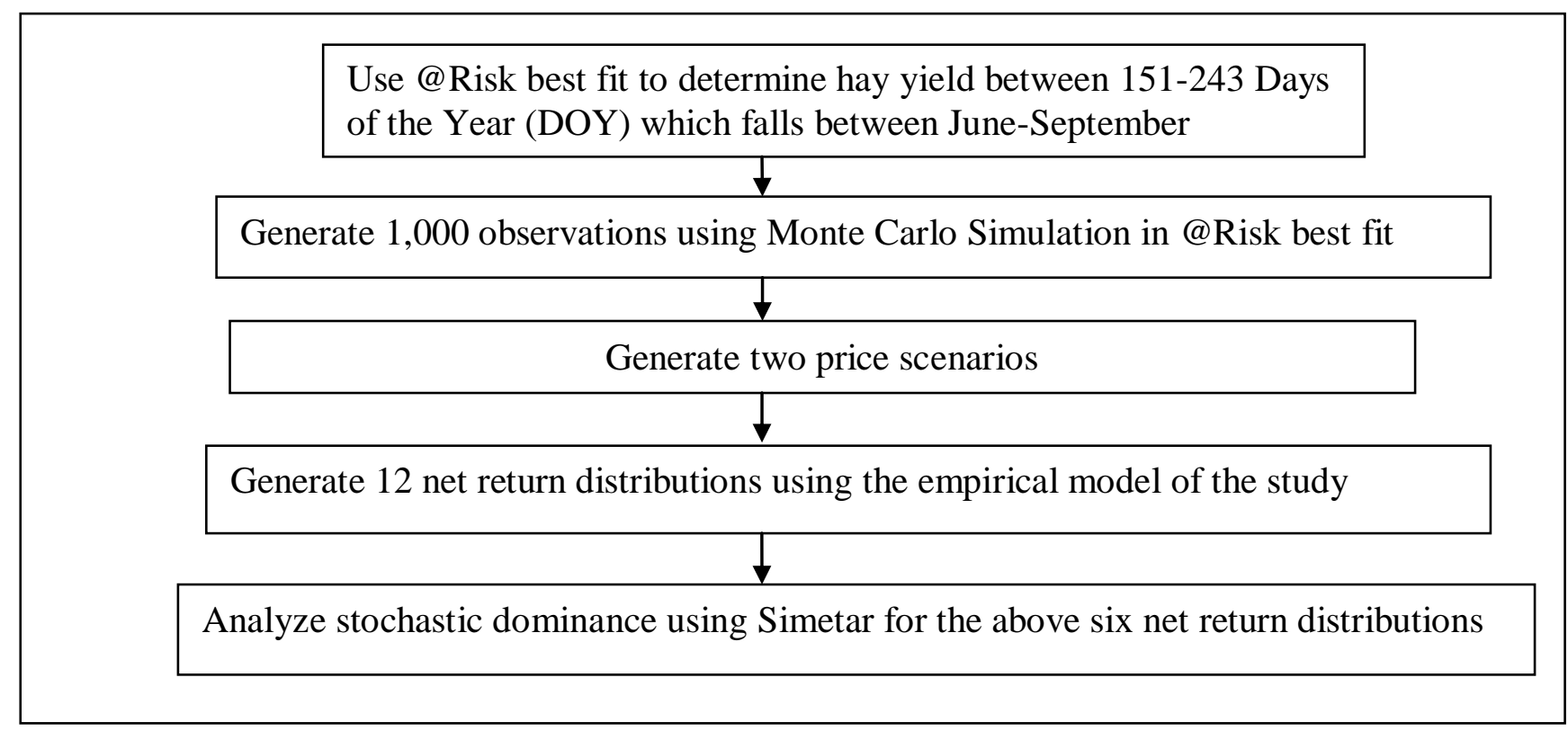

Figure 2-2. Methodology Flowchart of Essay II 


\subsection{Model Assumptions for Stochastic Dominance Analysis of Essay II:}

Table 2-1. illustrates the model assumptions to carry out stochastic dominance analysis in Essay

II.

Table 2-1.: Model Assumptions for Essay II

\begin{tabular}{|c|c|}
\hline $\begin{array}{l}\text { Model } \\
\text { Parameter(s) }\end{array}$ & Description(s) \\
\hline $\begin{array}{l}\text { Area to be } \\
\text { insured }\end{array}$ & 25 acres \\
\hline Interval(s) & $\begin{array}{l}\text { III and IV, correspond to Day of the Year (DOY) of 151-243 (June- } \\
\text { September), as this is the critical back grounding period for Grass fed } \\
\text { producers of West Virginia. This period falls in two intervals of Rainfall } \\
\text { insurance as marketed by RMA. } \\
\text { Interval III: June-July } \\
\text { Interval IV: August-September }\end{array}$ \\
\hline $\begin{array}{l}\text { Productivity } \\
\text { Factor }\end{array}$ & $\begin{array}{l}100 \% \text {, this factor has been determined by WVU Extension as the preferred } \\
\text { productivity level }\end{array}$ \\
\hline Location & $\begin{array}{l}\text { Allegheny County of PA, Grid } 24500 \text { and } 24800 \text {, this insurance has not been } \\
\text { introduced in WV as of yet, Allegheny county is nearest to WV where this } \\
\text { insurance being offered as part of the pilot program. }\end{array}$ \\
\hline Distribution & Hay yield distribution follows a Triangular Distribution as reported by Best Fit. \\
\hline $\begin{array}{l}\text { Coverage } \\
\text { Level }\end{array}$ & $70 \%, 75 \%, 80 \%, 85 \%$ and $90 \%$. \\
\hline Hay Price & $\begin{array}{l}\text { Hay price varies depending on quality, to capture this variability } 2 \text { scenarios: } \\
\$ 110 / \text { ton and } \$ 200 / \text { ton have been assumed which will take care of this price } \\
\text { variability and also this deems to be perfect with people associated with hay } \\
\text { business in West Virginia. }\end{array}$ \\
\hline $\begin{array}{l}\text { Type of Cost } \\
\text { Considered }\end{array}$ & Production cost has been eliminated from the model \\
\hline Case Studies & $\begin{array}{l}\text { A: Average Positive Payout } \\
\text { B: Average Negative Payout } \\
\text { It was important to build out these two scenarios, as this insurance will not } \\
\text { offer pay out every year. Positive and Negative pay outs are the averaged } \\
\text { positive and negative pay outs (indemnity minus premium) as calculated using } \\
60 \text { years of cumulative data from USDA/RMA Rainfall Insurance calculator } \\
\text { web page. }\end{array}$ \\
\hline
\end{tabular}




\subsection{Data and Data Analysis}

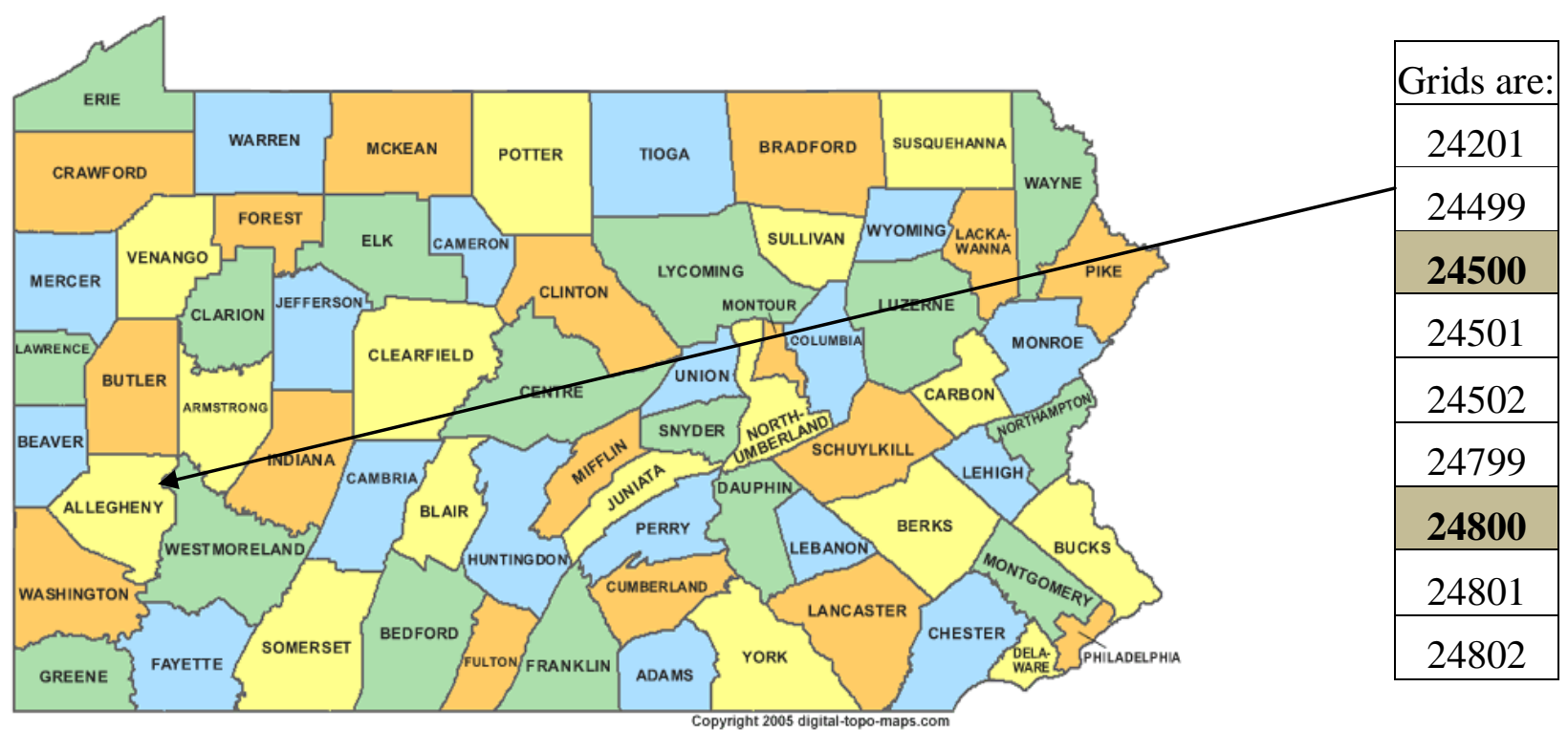

Figure 2-3.: PA Counties

The following table reports various sources of data used in this study.

Table 2-2: Data Sources for Essay II

\begin{tabular}{|l|l|}
\hline Variable & Data Source \\
\hline Hay Yield & $\begin{array}{l}\text { Since Rainfall Insurance is still not available for West Virginia, } \\
\text { hence Pennsylvania } \\
\text { Project Trial data of Allegheny county (since it is nearest to WV) } \\
\text { of WVU Extension Dept. was used. }\end{array}$ \\
\hline Hay Price & $\begin{array}{l}\text { Expert Consultation with Dr. Ed Rayburn of WVU Extension } \\
\text { Dept. }\end{array}$ \\
\hline $\begin{array}{l}\text { Rain fall Insurance } \\
\text { Indemnity/Premium }\end{array}$ & http://agforceusa.com/rma/ri/prf/dst \\
\hline Grid Locator & http://prfri-rma-map.tamu.edu/getGridId.aspx \\
\hline
\end{tabular}


Table 2-3.: Different Rainfall Insurance Choices available

\begin{tabular}{|c|c|c|}
\hline Choice(s) & Type & Significance \\
\hline I & No_Insur_PriceI & No Insurance, Price $\$ 200 /$ ton \\
\hline II & PriceI_I & $70 \%$ Coverage, Price $\$ 200 /$ ton \\
\hline III & PriceI_II & $75 \%$ Coverage, Price $\$ 200 /$ ton \\
\hline IV & PriceI_III & $80 \%$ Coverage, Price $\$ 200 /$ ton \\
\hline V & PriceI_IV & $85 \%$ Coverage, Price $\$ 200 /$ ton \\
\hline VI & PriceI_V & 90\% Coverage, Price $\$ 200 /$ ton \\
\hline VII & No_Insur_PriceII & No Insurance, Price $\$ 110 /$ ton \\
\hline VII & PriceII_I & $70 \%$ Coverage, Price $\$ 110 /$ ton \\
\hline IX & PriceII_II & $75 \%$ Coverage, Price $\$ 110 /$ ton \\
\hline X & PriceII_III & $80 \%$ Coverage, Price $\$ 110 /$ ton \\
\hline XI & PriceII_IV & $85 \%$ Coverage, Price $\$ 110 /$ ton \\
\hline XII & PriceII_V & $90 \%$ Coverage, Price $\$ 110 /$ ton \\
\hline
\end{tabular}

Table 2-4.: Net Indemnity Received after Paying Premiums for Rainfall Insurance for Pastures for Interval III of Grid 24500(Grid I)

\begin{tabular}{|c|l|l|l|l|l|}
\hline Coverage Level & $70 \%$ & $75 \%$ & $80 \%$ & $85 \%$ & $90 \%$ \\
\hline Average net $^{1}$ indemnity $^{1}$ & $\$ 1.87$ & $\$ 1.59$ & $\$ 1.23$ & $\$ 1.01$ & $\$ 0.75$ \\
& & & & & \\
\hline Average Positive Payout & $\$ 7.71$ & $\$ 7.63$ & $\$ 7.19$ & $\$ 6.99$ & $\$ 5.61$ \\
\hline Probability Positive Payout & $37 \%$ & $31 \%$ & $25 \%$ & $20 \%$ & $19 \%$ \\
\hline Average Negative Payout & $-\$ 1.60$ & $-\$ 1.60$ & $-\$ 0.80$ & $-\$ 0.51$ & $-\$ 0.36$ \\
\hline Probability Negative Payout & $63 \%$ & $69 \%$ & $75 \%$ & $80 \%$ & $81 \%$ \\
\hline Maximum Net Indemnity & $\$ 21.53$ & $\$ 19.98$ & $\$ 18.23$ & $\$ 16.49$ & $\$ 14.61$ \\
\hline Minimum Net Indemnity & $-\$ 1.61$ & $-\$ 1.12$ & $-\$ 0.82$ & $-\$ 0.52$ & $-\$ 0.36$ \\
\hline
\end{tabular}

Where

Average net indemnity: The average indemnity less premium.

Average Positive Payout: The average indemnity less premium when the net indemnity is greater than zero.

Probability Positive Payout: The probability of receiving a net indemnity that is greater than zero for 60 years of weather data.

Average Negative Payout: The average indemnity less premium when the net indemnity is less than zero for 60 years of weather data. 
Probability Negative Payout: The probability of receiving a net indemnity that is less than zero for 60 years of weather data.

Maximum Net Indemnity: The largest indemnity less premium for 60 years of weather data.

Minimum Net Indemnity: The smallest indemnity less premium for the 60 years of weather data.

Data Source for table 2.3-2.6 is the USDA/RMA online decision support system available at:

rma.usda.gov/policies/pasture range forage.

Table 2-5.: Net Indemnity Received after Paying Premiums for Rainfall Insurance for Pastures for Interval IV of Grid 24500 (Grid I)

\begin{tabular}{|c|l|l|l|l|l|}
\hline Coverage Level & $70 \%$ & $75 \%$ & $80 \%$ & $85 \%$ & $90 \%$ \\
\hline Average net indemnity & $\$ 2.12$ & $\$ 1.85$ & $\$ 1.46$ & $\$ 1.18$ & $\$ 0.78$ \\
\hline Average Positive Payout & $\$ 8.99$ & $\$ 8.32$ & $\$ 6.61$ & $\$ 4.97$ & $\$ 4.27$ \\
\hline Probability Positive Payout & $37 \%$ & $34 \%$ & $34 \%$ & $34 \%$ & $27 \%$ \\
\hline Average Negative Payout & $-\$ 1.96$ & $-\$ 1.47$ & $-\$ 1.18$ & $-\$ 0.77$ & $-\$ 0.52$ \\
\hline Probability Negative Payout & $63 \%$ & $66 \%$ & $66 \%$ & $66 \%$ & $73 \%$ \\
\hline Maximum Net Indemnity & $\$ 16.33$ & $\$ 14.85$ & $\$ 13.13$ & $\$ 11.50$ & $\$ 9.70$ \\
\hline Minimum Net Indemnity & $-\$ 2.07$ & $-\$ 1.51$ & $-\$ 1.18$ & $-\$ 0.77$ & $-\$ 0.52$ \\
\hline
\end{tabular}

Table 2-6.: Net Indemnity Received after Paying Premiums for Rainfall Insurance for Pastures for Interval III of Grid 24800 (Grid II)

\begin{tabular}{|c|l|l|l|l|l|}
\hline Coverage Level & $70 \%$ & $75 \%$ & $80 \%$ & $85 \%$ & $90 \%$ \\
\hline Average net indemnity & $\$ 1.94$ & $\$ 1.72$ & $\$ 1.38$ & $\$ 1.17$ & $\$ 0.82$ \\
\hline Average Positive Payout & $\$ 9.60$ & $\$ 9.12$ & $\$ 7.38$ & $\$ 5.75$ & $\$ 4.99$ \\
\hline Probability Positive Payout & $34 \%$ & $31 \%$ & $31 \%$ & $31 \%$ & $25 \%$ \\
\hline Average Negative Payout & $-\$ 1.99$ & $-\$ 1.53$ & $-\$ 1.25$ & $-\$ 0.84$ & $-\$ 0.60$ \\
\hline Probability Negative Payout & $66 \%$ & $69 \%$ & $69 \%$ & $69 \%$ & $75 \%$ \\
\hline Maximum Net Indemnity & $\$ 22.25$ & $\$ 20.77$ & $\$ 19.03$ & $\$ 17.40$ & $\$ 15.59$ \\
\hline Minimum Net Indemnity & $-\$ 2.12$ & $-\$ 1.56$ & $-\$ 1.25$ & $-\$ 0.84$ & $-\$ 0.60$ \\
\hline
\end{tabular}


Table 2-7.: Net Indemnity Received after Paying Premiums for Rainfall Insurance for Pastures for Interval IV of Grid 24800 (Grid II)

\begin{tabular}{|c|l|l|l|l|l|}
\hline Coverage Level & $70 \%$ & $75 \%$ & $80 \%$ & $85 \%$ & $90 \%$ \\
\hline Average net indemnity & $\$ 2.35$ & $\$ 1.95$ & $\$ 1.34$ & $\$ 1.08$ & $\$ 0.78$ \\
& & & & & \\
\hline Average Positive Payout & $\$ 10.16$ & $\$ 8.32$ & $\$ 6.61$ & $\$ 4.97$ & $\$ 3.60$ \\
\hline Probability Positive Payout & $38 \%$ & $33 \%$ & $33 \%$ & $33 \%$ & $25 \%$ \\
\hline Average Negative Payout & $-\$ 1.96$ & $-\$ 1.47$ & $-\$ 1.18$ & $-\$ 0.77$ & $-\$ 0.52$ \\
\hline Probability Negative Payout & $64 \%$ & $67 \%$ & $67 \%$ & $67 \%$ & $75 \%$ \\
\hline Maximum Net Indemnity & $\$ 16.33$ & $\$ 14.85$ & $\$ 13.13$ & $\$ 11.50$ & $\$ 9.70$ \\
\hline Minimum Net Indemnity & $-\$ 2.07$ & $-\$ 1.51$ & $-\$ 1.18$ & $-\$ 0.77$ & $-\$ 0.52$ \\
\hline
\end{tabular}

Table 2-8.: Descriptive Statistics of Net Return Distribution Grid 24500

Average Positive Payout

\begin{tabular}{|c|r|r|r|r|r|r|c|c|c|c|c|c|}
\hline Choice(s) & \multicolumn{1}{|c|}{ I } & \multicolumn{1}{c|}{ II } & \multicolumn{1}{c|}{ III } & \multicolumn{1}{c|}{ IV } & \multicolumn{1}{c|}{ V } & \multicolumn{1}{c|}{ VI } & \multicolumn{1}{c|}{ VII } & VII & IX & X & XI & XII \\
\hline Average(\$) & 346.26 & 362.96 & 362.21 & 360.06 & 358.22 & 356.14 & 629.56 & 646.26 & 645.51 & 643.36 & 641.52 & 639.44 \\
\hline Std.Dev(\$) & 62.81 & 62.81 & 62.81 & 62.81 & 62.81 & 62.81 & 114.20 & 114.20 & 114.20 & 114.20 & 114.20 & 114.20 \\
\hline Min.(\$) & 195.60 & 212.30 & 211.55 & 209.40 & 207.56 & 205.48 & 355.64 & 372.34 & 371.59 & 369.44 & 367.60 & 365.52 \\
\hline Max.(\$) & 492.09 & 508.79 & 508.04 & 505.89 & 504.05 & 501.97 & 894.71 & 911.41 & 910.66 & 908.51 & 906.67 & 904.59 \\
\hline
\end{tabular}

Average Negative Payout

\begin{tabular}{|c|r|r|r|r|r|r|r|c|c|c|c|c|}
\hline Choice(s) & \multicolumn{1}{|c|}{ I } & \multicolumn{1}{c|}{ II } & \multicolumn{1}{c|}{ III } & \multicolumn{1}{c|}{ IV } & \multicolumn{1}{c|}{ V } & \multicolumn{1}{c|}{ VI } & \multicolumn{1}{c|}{ VII } & \multicolumn{1}{c|}{ VII } & IX & X & XI & XII \\
\hline Average(\$) & 346.26 & 342.70 & 343.19 & 344.28 & 344.98 & 345.38 & 629.22 & 626.00 & 626.49 & 627.58 & 628.28 & 628.68 \\
\hline Std.Dev(\$) & 62.81 & 62.81 & 62.81 & 62.81 & 62.81 & 62.81 & 114.49 & 114.20 & 114.20 & 114.20 & 114.20 & 114.20 \\
\hline Min.(\$) & 195.60 & 192.04 & 192.53 & 193.62 & 194.32 & 194.72 & 355.64 & 352.08 & 352.57 & 353.66 & 354.36 & 354.76 \\
\hline Max.(\$) & 492.09 & 488.53 & 489.02 & 490.11 & 490.81 & 491.21 & 894.71 & 891.15 & 891.64 & 892.73 & 893.43 & 893.83 \\
\hline
\end{tabular}


Table 2-9.: Descriptive Statistics of Net Return Distribution Grid 24800

Average Positive Payout

\begin{tabular}{|l|r|r|r|r|r|r|r|r|r|r|r|r|}
\hline Choice(s) & I & II & III & IV & V & VI & VII & VII & IX & X & XI & XII \\
\hline Average(\$) & 346.26 & 364.85 & 363.70 & 360.25 & 356.98 & 355.52 & 629.59 & 648.15 & 647.00 & 643.55 & 640.28 & 638.82 \\
\hline Std.Dev(\$) & 62.81 & 62.81 & 62.81 & 62.81 & 62.81 & 62.81 & 114.19 & 114.20 & 114.20 & 114.20 & 114.20 & 114.20 \\
\hline Min.(\$) & 195.60 & 214.19 & 213.04 & 209.59 & 206.32 & 204.86 & 355.64 & 374.23 & 373.08 & 369.63 & 366.36 & 364.90 \\
\hline Max.(\$) & 492.09 & 510.68 & 509.53 & 506.08 & 502.81 & 501.35 & 894.71 & 913.30 & 912.15 & 908.70 & 905.43 & 903.97 \\
\hline
\end{tabular}

Average Negative Payout

\begin{tabular}{|l|r|r|r|r|r|r|r|r|r|r|r|r|}
\hline Choice(s) & I & II & III & IV & V & VI & VII & VII & IX & X & XI & XII \\
\hline Average(\$) & 346.26 & 342.31 & 343.26 & 343.83 & 344.65 & 345.14 & 629.59 & 625.61 & 626.56 & 627.13 & 627.95 & 628.44 \\
\hline Std.Dev(\$) & 62.81 & 62.81 & 62.81 & 62.81 & 62.81 & 62.81 & 114.19 & 114.20 & 114.20 & 114.20 & 114.20 & 114.20 \\
\hline Min.(\$) & 195.60 & 191.65 & 192.60 & 193.17 & 193.99 & 194.48 & 355.64 & 351.69 & 352.64 & 353.21 & 354.03 & 354.52 \\
\hline Max.(\$) & 492.09 & 488.14 & 489.09 & 489.66 & 490.48 & 490.97 & 894.71 & 890.76 & 891.71 & 892.28 & 893.10 & 893.59 \\
\hline
\end{tabular}


2.7. Estimation and Results

2.7.1. Grid 24500

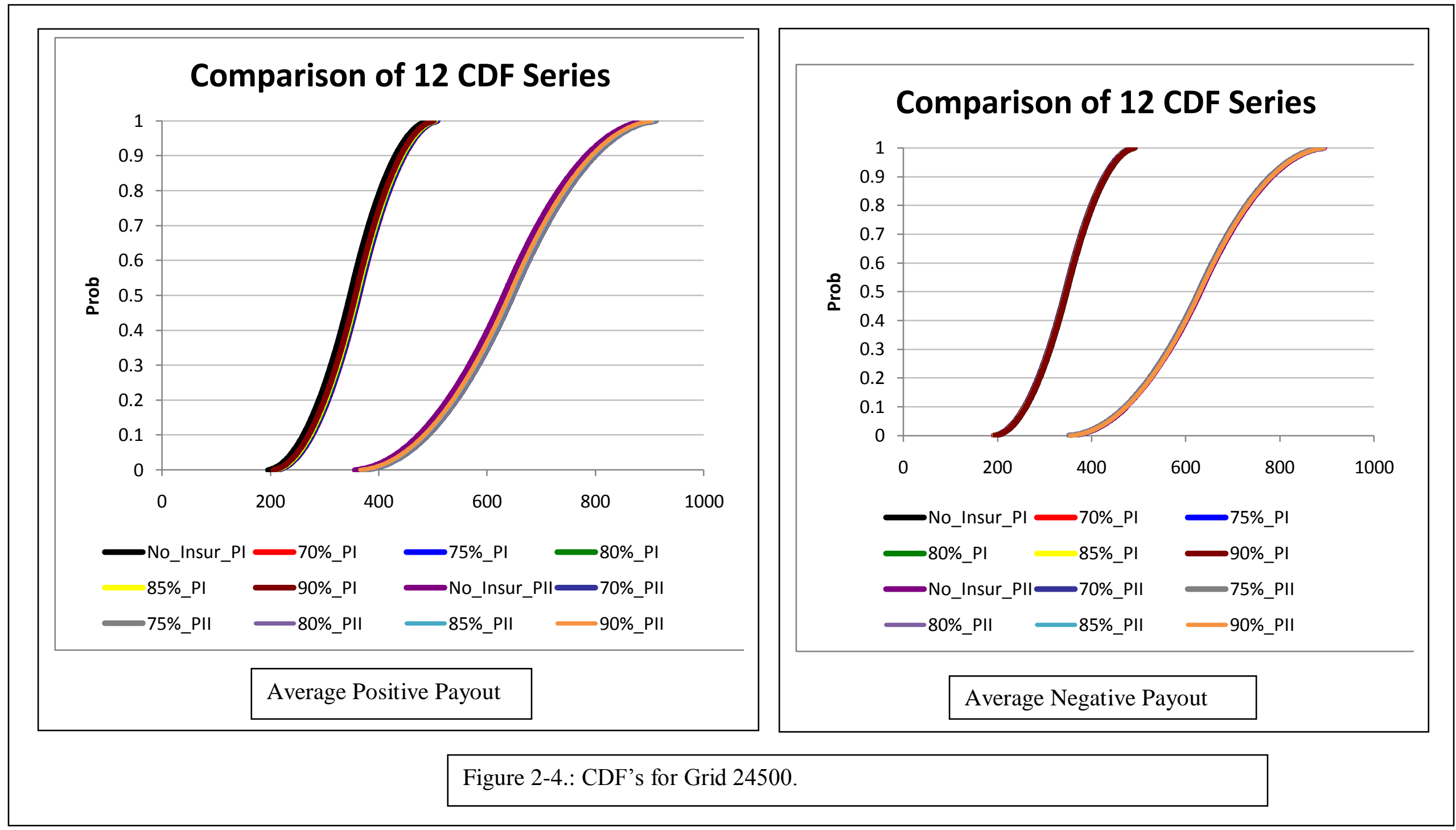


Table 2-10.: Risk Ranking for Grid 24500

\begin{tabular}{|c|c|c|c|c|c|}
\hline \multicolumn{3}{|c|}{ Average Positive Payout } & \multicolumn{3}{|c|}{ Average Negative Payout } \\
\hline $\begin{array}{c}\text { Risk } \\
\text { Ranking }\end{array}$ & $\begin{array}{l}\text { Insurance } \\
\text { Choice }\end{array}$ & $\begin{array}{c}\text { Level of } \\
\text { Preference by } \\
\text { FSD }\end{array}$ & $\begin{array}{c}\text { Risk } \\
\text { Ranking }\end{array}$ & Insurance Choice & $\begin{array}{c}\text { Level of Preference } \\
\text { by FSD }\end{array}$ \\
\hline 1 & 70\%_PII & Most Preferred & 1 & No_Insur_PII & Most Preferred \\
\hline 2 & 75\%_PII & $\begin{array}{l}\text { 2nd Most } \\
\text { Preferred }\end{array}$ & 2 & 90\%_PII & 2nd Most Preferred \\
\hline 3 & 80\%_PII & $\begin{array}{l}\text { 3rd Most } \\
\text { Preferred }\end{array}$ & 3 & 85\%_PII & 3rd Most Preferred \\
\hline 4 & $85 \% \_$PII & $\begin{array}{l}\text { 4th Most } \\
\text { Preferred }\end{array}$ & 4 & $80 \% \_$PII & 4th Most Preferred \\
\hline 5 & 90\%_PII & $\begin{array}{l}\text { 5th Most } \\
\text { Preferred }\end{array}$ & 5 & 75\%_PII & 5th Most Preferred \\
\hline 6 & No_Insur_PII & $\begin{array}{l}\text { 6th Most } \\
\text { Preferred }\end{array}$ & 6 & $70 \% \_$PII & 6th Most Preferred \\
\hline 7 & 70\%_PI & $\begin{array}{l}\text { 7th Most } \\
\text { Preferred }\end{array}$ & 7 & No_Insur_PI & 7th Most Preferred \\
\hline 8 & 75\%_PI & $\begin{array}{l}\text { 8th Most } \\
\text { Preferred }\end{array}$ & 8 & 90\%_PI & 8th Most Preferred \\
\hline 9 & 80\%_PI & $\begin{array}{l}\text { 9th Most } \\
\text { Preferred }\end{array}$ & 9 & $85 \% \_$PI & 9th Most Preferred \\
\hline 10 & 85\%_PI & $\begin{array}{l}\text { 10th Most } \\
\text { Preferred }\end{array}$ & 10 & 80\%_PI & 10th Most Preferred \\
\hline 11 & 90\%_PI & $\begin{array}{l}\text { 11th Most } \\
\text { Preferred }\end{array}$ & 11 & 75\%_PI & 11th Most Preferred \\
\hline 12 & No_Insur_PI & $\begin{array}{l}\text { 12th Most } \\
\text { Preferred }\end{array}$ & 12 & 70\%_PI & 12th Most Preferred \\
\hline
\end{tabular}




\subsubsection{Grid 24800}
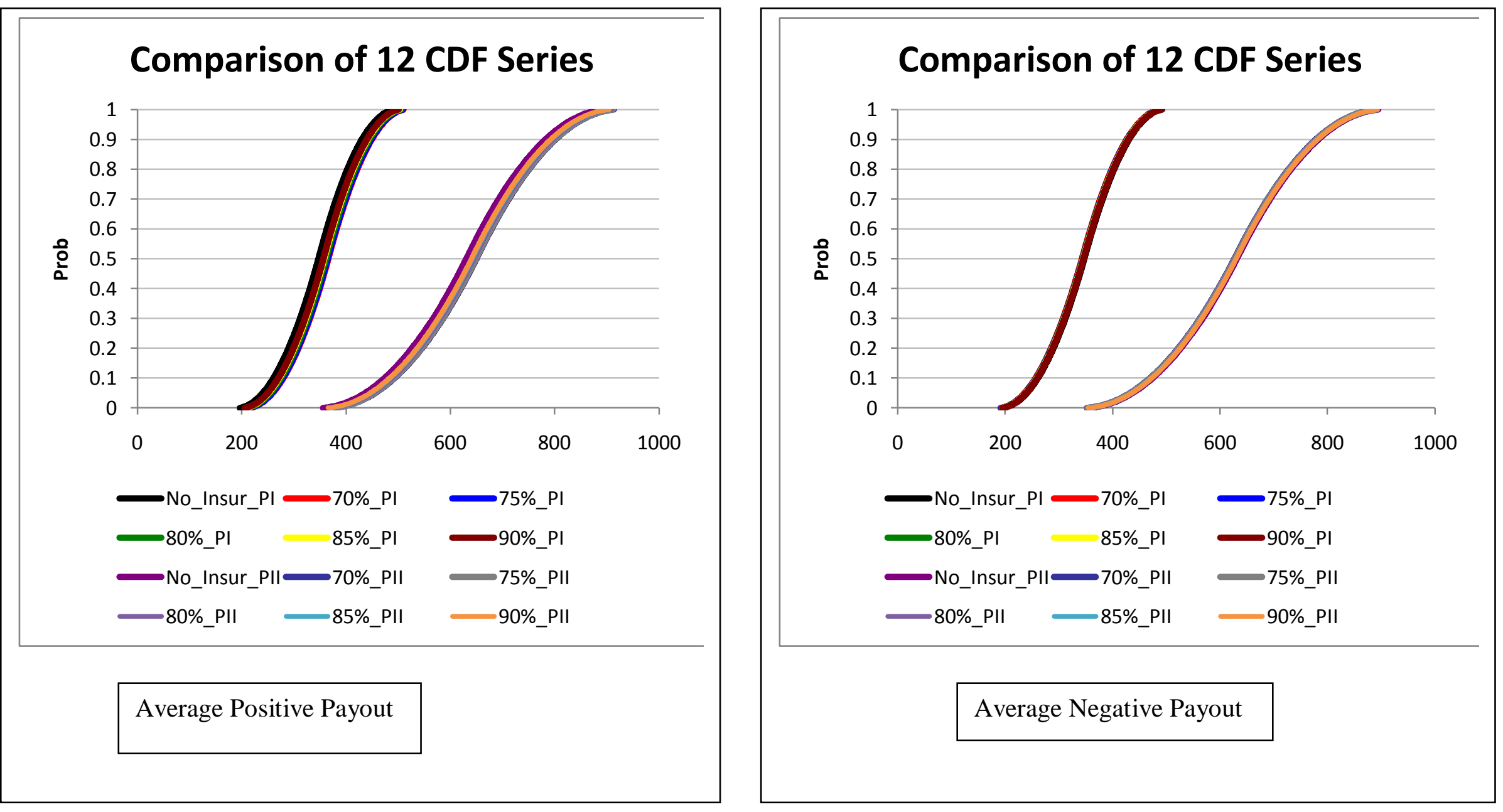

Figure 2-5.: CDF's for Grid 24800 
Table 2-11.: FSD Risk Rankings for Grid 24500

\begin{tabular}{|c|c|c|c|c|c|}
\hline \multicolumn{3}{|c|}{ Average Positive Payout } & \multicolumn{3}{|c|}{ Average Negative Payout } \\
\hline $\begin{array}{c}\text { Risk } \\
\text { Ranking }\end{array}$ & $\begin{array}{c}\text { Insurance } \\
\text { Choice }\end{array}$ & $\begin{array}{c}\text { Level of } \\
\text { Preference by } \\
\text { FSD }\end{array}$ & $\begin{array}{c}\text { Risk } \\
\text { Ranking }\end{array}$ & Insurance Choice & $\begin{array}{c}\text { Level of Preference } \\
\text { by FSD }\end{array}$ \\
\hline 1 & $70 \% \_$PII & Most Preferred & 1 & No_Insur_PII & Most Preferred \\
\hline 2 & 75\%_PII & $\begin{array}{l}\text { 2nd Most } \\
\text { Preferred }\end{array}$ & 2 & 90\%_PII & 2nd Most Preferred \\
\hline 3 & 80\%_PII & $\begin{array}{l}\text { 3rd Most } \\
\text { Preferred }\end{array}$ & 3 & 85\%_PII & 3rd Most Preferred \\
\hline 4 & 85\%_PII & $\begin{array}{l}\text { 4th Most } \\
\text { Preferred }\end{array}$ & 4 & 80\%_PII & 4th Most Preferred \\
\hline 5 & 90\%_PII & $\begin{array}{l}\text { 5th Most } \\
\text { Preferred }\end{array}$ & 5 & 75\%_PII & 5th Most Preferred \\
\hline 6 & No_Insur_PII & $\begin{array}{l}\text { 6th Most } \\
\text { Preferred } \\
\end{array}$ & 6 & 70\%_PII & 6th Most Preferred \\
\hline 7 & 70\%_PI & $\begin{array}{l}\text { 7th Most } \\
\text { Preferred }\end{array}$ & 7 & No_Insur_PI & 7th Most Preferred \\
\hline 8 & 75\%_PI & $\begin{array}{l}\text { 8th Most } \\
\text { Preferred } \\
\end{array}$ & 8 & 90\%_PI & 8th Most Preferred \\
\hline 9 & 80\%_PI & $\begin{array}{l}\text { 9th Most } \\
\text { Preferred } \\
\end{array}$ & 9 & $85 \%$ PI & 9th Most Preferred \\
\hline 10 & $85 \% \_P I$ & $\begin{array}{l}\text { 10th Most } \\
\text { Preferred }\end{array}$ & 10 & $80 \%$ PI & 10th Most Preferred \\
\hline 11 & 90\%_PI & $\begin{array}{l}\text { 11th Most } \\
\text { Preferred }\end{array}$ & 11 & 75\%_PI & 11th Most Preferred \\
\hline 12 & No_Insur_PI & $\begin{array}{l}\text { 12th Most } \\
\text { Preferred }\end{array}$ & 12 & 70\%_PI & 12th Most Preferred \\
\hline
\end{tabular}

\subsection{Climate Effects and Rainfall Index Insurance Purchase Decision}

As we have seen in the previous sections, the rainfall insurance premiums and estimates of potential indemnity payments are based on past averages of rainfall as determined for two month intervals under all climatic conditions. However, indemnifying 2-month intervals based on alternative climate phases, in particular those associated with the El Nino Southern Oscillation (ENSO, described in detail in Essay III), may change the bimonthly intervals that, if insured, would provide the largest potential insurance protection level to the farm operation. The ENSO impact on the bi-monthly rainfall insurance is likely to be more pronounced than the impact on 
annual yield/revenue insurance (1) because of the nature of the insured variable and (2) because ENSO phases, particularly in the Southeastern U.S., affect regional climate most strongly in late fall and winter (Jewson, 2002) . Rainfall Index (RI) insurance actuarial rates are set by RMA/USDA and are based on pooled climate data going back to 1948. The ENSO is not currently considered in setting insurance premium rates. ENSO/EL Niño will be discussed in detail in section 3.13. A study by Novak et al. (2008) analyzes the expected payoff of the RI insurance for two month periods based on probabilities of rainfall shortage during alternative climate phases (El Nino, La Nina, and Neutral) at 4 Alabama Agricultural Experiment Station sites under varying coverage levels. Policy makers and producers are expected to benefit from results that show the varying effects of climate ENSO phases on maximum likely payouts from indemnifying 2 2-month periods. Implications from this study also ask for setting actuarial rates based on specific ENSO phase.

\subsection{Conclusion}

Rainfall insurance has been introduced in a pilot manner since 2008 as part of pastureland, range and forage insurance program by USDA-RMA. It is important for the risk managers to understand that this insurance may or may not result in indemnity payments for each year. The above analysis was carried out using 60 years of pooled actuarial data. It can be seen that under negative payout scenarios, the most preferred choice is no insurance and under positive pay out scenarios, $70 \%$ coverage is the most preferred scenario reported for the hay producers of these two particular grids in Pennsylvania. Another point worth mentioning is that the current indemnities do not take into account the climatic variability associated with ENSO/El Nino phases. 


\section{List of References}

\section{A. Journal Articles}

Novak, J. and D. Nadolnyak. 2009. "Climatic Effects in Rainfall Insurance Purchase Decision". Selected paper, presented at the 2009 annual meeting of the Southern Agricultural Economics Association. Atlanta, GA.

\section{B. Books}

Dionne, Georges and S.E. Harrington, editors, 1992, Foundations of Insurance Economics: Readings in Economics and Finance. Kluwer, Boston.

Jewson, Stephen and A.Brix, 2005. Weather Derivative Valuation: The Meteorological, Statistical, Financial and Mathematical Foundations. Cambridge University Press, Cambridge.

\section{Websites}

Fact sheet of European Commission on Agriculture; Available at:

http://ec.europa.eu/agriculture/fin/clearance/factsheet_en.pdf.

Grid Locator; Available at: http://prfri-rma-map.tamu.edu/getGridId.aspx.

Premium Calculator; Available at: http://agforceusa.com/rma/ri/prf/dst.

USDA/National Agricultural Statistics Service (NASS). 2007. Selected data. Available web at www.nass.usda.gov. 


\section{CHAPTER 3}

\section{STRUCTURING/MODELLING A PRECIPITATION OPTION (CALL/PUT) FOR WEST VIRGINIA}

If you wish to control the future, study the past...

- Confucius

\subsection{Introduction}

Weather derivatives are financial instruments that can be used by organizations or individuals as part of a risk- management strategy to reduce risk associated with adverse or unexpected weather conditions. The difference from other derivatives (i.e. options) is that the underlying asset (e.g. rain/temperature/snow) has no direct value to price the weather derivative. Farmers can use weather derivatives as protection against poor harvests caused by drought or frost; theme parks may want to insure against rainy weekends during peak summer seasons; and gas and power companies may use heating degree days (HDD) or cooling degree days (CDD) contracts to smooth their annual earnings. A sports event managing company may wish to hedge its losses by entering into a weather derivative contract in case it rains the day of the sporting event and fewer tickets are sold. HDD is one of the most common types of weather derivative. An HDD contract could be for the November to March period, for each day when the temperature falls below $65^{0} \mathrm{~F}$ $\left(18^{0} \mathrm{C}\right)$ one can keep a cumulative count of the difference between $65^{\circ} \mathrm{F}\left(18^{0} \mathrm{C}\right)$ and the average daily temperature. Depending upon whether the option is a put option or a call option, a set amount will be paid out per heating degree day that the actual count differs from the strike, where the strike is the trigger rate of the index (heating degree days in this example) as set in the option contract. The first weather derivative contract was created in July 1996, when Aquila Energy (an energy trading firm based in Kansas City, MO) structured a dual-commodity hedge 
for Consolidated Edison Co (ConEd), NY (ConEd, 1996). The transaction involved ConEd's purchase of electric power from Aquila for the month of August of that year (speedwell.com). The price of the power was agreed on, but a weather clause was also embedded into the contract. This clause stipulated that Aquila would pay ConEd a rebate if August turned out to be a cooler month than expected. The measurement of this was referenced using CDD measured at New York City's Central Park weather station. If the total expected number of CDDs was found in the range from 0 to $10 \%$, which is below the expected number of 320 , the company will not receive a discount in power price. However, if the total expected CDDs was found to be 11 to $20 \%$ below the normal expected value, Con Ed would receive a $\$ 16,000$ discount. Other discounted levels were calculated for even greater departures from the expected CDD.

After that humble beginning, weather derivatives slowly began to trade over-the-counter (OTC) in 1997. As the market for these products grew, the Chicago Mercantile Exchange (CME) introduced the first exchange-traded weather futures contracts (and the corresponding options) in 1999 (cmegroup.com/trading/weather/). The CME currently trades weather derivative contracts for twenty-four cities in the United States, ten in Europe, six in Canada and three in Japan. Most of these contracts track CDD or HDD, but recent additions track frost days in the Netherlands and monthly/seasonal snowfall in Boston and New York. A major early pioneer in weather derivatives was Enron Corporation, through its Enron Online unit (Climetrix.com). Although agriculture suffers most from weather related risk, most of these weather related derivative deals have been structured for the energy sector to date. This essay discusses the concept of weather derivatives as potentially applied in agriculture, the mechanism of the option based contracts, and the structure of weather contracts based on temperature and rainfall. Also, this essay includes 
a literature review, rainfall simulation, and a pricing exercise utilizing both burn and Monte Carlo simulation in consecutive sections.

\subsection{How Weather Derivatives Work}

Weather derivatives are either bought or sold. The buyer wants to reduce risk from extreme weather conditions by transferring some of the risk to the seller.

Table 3-1: How weather derivatives work

\begin{tabular}{|l|l|}
\hline \multicolumn{1}{|c|}{ Buyer } & \multicolumn{1}{c|}{ Seller } \\
\hline Pays the seller a premium to assume the risk & Takes premium from buyer in exchange \\
\hline If Extreme Conditions Occur: & \\
\hline Receives the insured amount (or the contract amount) & Pays the insured (or the contract) amount \\
to cover business losses & \\
\hline If Extreme Conditions Do Not Occur: & \\
\hline Does not lose business. Derivative does not pay off & Premium paid by buyer becomes profit. \\
\hline
\end{tabular}

\subsection{Derivatives, Insurance and Reinsurance: A Review of Risk Management Tools}

It is important to understand the subtle difference between the three types of risk management tools namely derivatives, insurance and reinsurance. These tools may appear to work as similar instruments in many situations; however, a few subtle differences exist. Native to both the notion of derivatives and insurance contracts is the idea of contingency. Thus, both tools are generally viewed as contingent claims, whose payoffs are dependent on occurrence of a set of outlined 
provisions. Moreover, insurance contracts and derivatives are means for individuals to transfer their risk (i.e. risk-sharing). Insurable interest forms the basis of most, if not all, insurance contracts. Insurable interest means that a policyholder has a right to a consideration when a loss or damage to life, property, etc., has resulted and the policyholder suffering a financial loss.

It is true from a mathematical and financial structure point of view that there is not much difference, however, some differences exist although they do not always hold exactly. For example, a set of weather derivatives traded via exchanges (e.g. Chicago Mercantile Exchange) has standardized parameters and contract sizes. This creates a market, and subsequently fair price discovery takes place where traders can act on mispricing of other derivatives. Insurance, on the other hand, is typically tailor made and so is the price. The insurance might reference a specific asset (industrial asset for business insurance, home for property insurance). Another difference is that insurance is event-based, while weather derivatives settle on publicly available weather measurement data recorded at a referenced weather station. Also, weather derivatives cover lowrisk, high-probability events. Weather insurance, on the other hand, typically covers high-risk, low-probability events. For example, a company might use a weather derivative to hedge against a winter that forecasters think will be $5^{\circ} \mathrm{F}$ warmer than the historical average (a low-risk, highprobability event). In this case, the company knows its revenues would be affected by that kind of weather. But the same company would most likely purchase an insurance policy for protection against damage caused by a flood or hurricane (high-risk, low-probability events).

Reinsurance is purchased (Hull, 2007) by an insurance company (insurer such as Travelers) from a "reinsurer" (e.g. Swiss Re) as a means to transfer risk (loss or losses). The reinsurer and the insurer enter into a reinsurance agreement which details the basis in which the 
reinsurer will pay the insurer's losses (excess of loss or proportional). The reinsurer is paid a reinsurance premium by the insurer.

A specific example is as follows: Let us consider that Travelers is issuing thousands of policies. If Travelers sells one thousand policies, each with a \$1 million policy limit (the amount Travelers could pay on the policy), theoretically, Travelers could lose $\$ 1$ million on each policy - for a total of $\$ 1$ billion. It may be better to pass some potential risk to a reinsurance company as this will create financial stability. Usually insurance companies pass off their riskiest portfolio of insurance policies to the reinsurance firms.

\subsection{Evolution of Weather Market}

The weather market in the U.S. traces its roots to deregulation of the U.S. energy industry which took place in the late 1990s. Variability in weather conditions had always been recognized as one of the most significant factors affecting energy consumption, however, the effects of unpredictable seasonal weather patterns had previously been absorbed and managed within a regulated, monopoly environment (Climetrix.com). With deregulation, the various participants in the process of producing, marketing and delivering energy to U.S. households and businesses were left to confront weather as a new and significant risk to their bottom line (wrma.org). Beyond the obvious initial applications of weather derivatives to hedge energy risk, the market has expanded to address a wide array of weather risks faced by numerous other industry sectors.

The CME Group carved out an important niche in the weather derivatives market, but it has taken a long time for that opportunity to emerge. In 1999, this exchange launched a series of exchange traded futures and options based on temperature indices for various U.S. cities, but volume did not reach meaningful levels until 2003 (cmegroup.com/trading/weather/). Healthy growth came only when the exchange began offering clearing services for these transactions, which currently account for $91 \%$ of volume. Today, the exchange offers contracts on 
temperature and precipitation indices in 24 cities in the U.S, six in Canada, ten in Europe and two in Asia-Pacific. As can be seen in figure 3-2 volume has grown from 798,000 contracts in 2006 to nearly a million in 2007, with notional or contract value in dollars in 2007 equaling about $\$ 18$ billion (cmegroup.com). In 2007-08, this volume has been depressed by the turmoil in the financial markets and the general economic contraction, but not as much as other sectors of the futures industry (cmegroup.com). Total volume for January through November 2008 was 737,506 contracts, down by $16 \%$ from the prior year. Open interest was 168,542 contracts, down $35 \%$ from 2007. But some industry observers think that the weather risk market will be boosted by the Obama administration's proposed emphasis on wind power and other renewable energy solutions, which are highly dependent on the weather. Also, the use of a clearinghouse (e.g. $\mathrm{CME}$ ) to reduce counterparty risk as opposed to over the counter derivatives will make this investment more attractive.

The demand for weather derivatives mostly comes from the energy sector which accounts for more than half of the total weather derivatives business. This sector is followed by the construction industry and agriculture, according to the Weather Risk Management Association (wrma.org). Also, while the use of weather derivatives in the energy sector is more common than in any other sector, just $35 \%$ of energy companies manage their exposure to weather through the use of either off-exchange (OTC) or exchange-traded weather derivatives, according to the CME/Storm exchange, a provider of weather-related financial risk and information services (artemis.bm/blog). However, this volume is minimal compared to other types of derivatives. Figure 3-3 shows the West Texas Intermediate (WTI) (a type of crude oil) futures contracts traded at The New York Mercantile Exchange (NYMEX) and Interncontinetal Exchange (ICE) during 2006-07. This is in the order of millions compared to thousands of weather derivatives 
contracts. Figure 3-4 shows the total volume of transactions of different types of derivative contracts including interest rate and CDS .

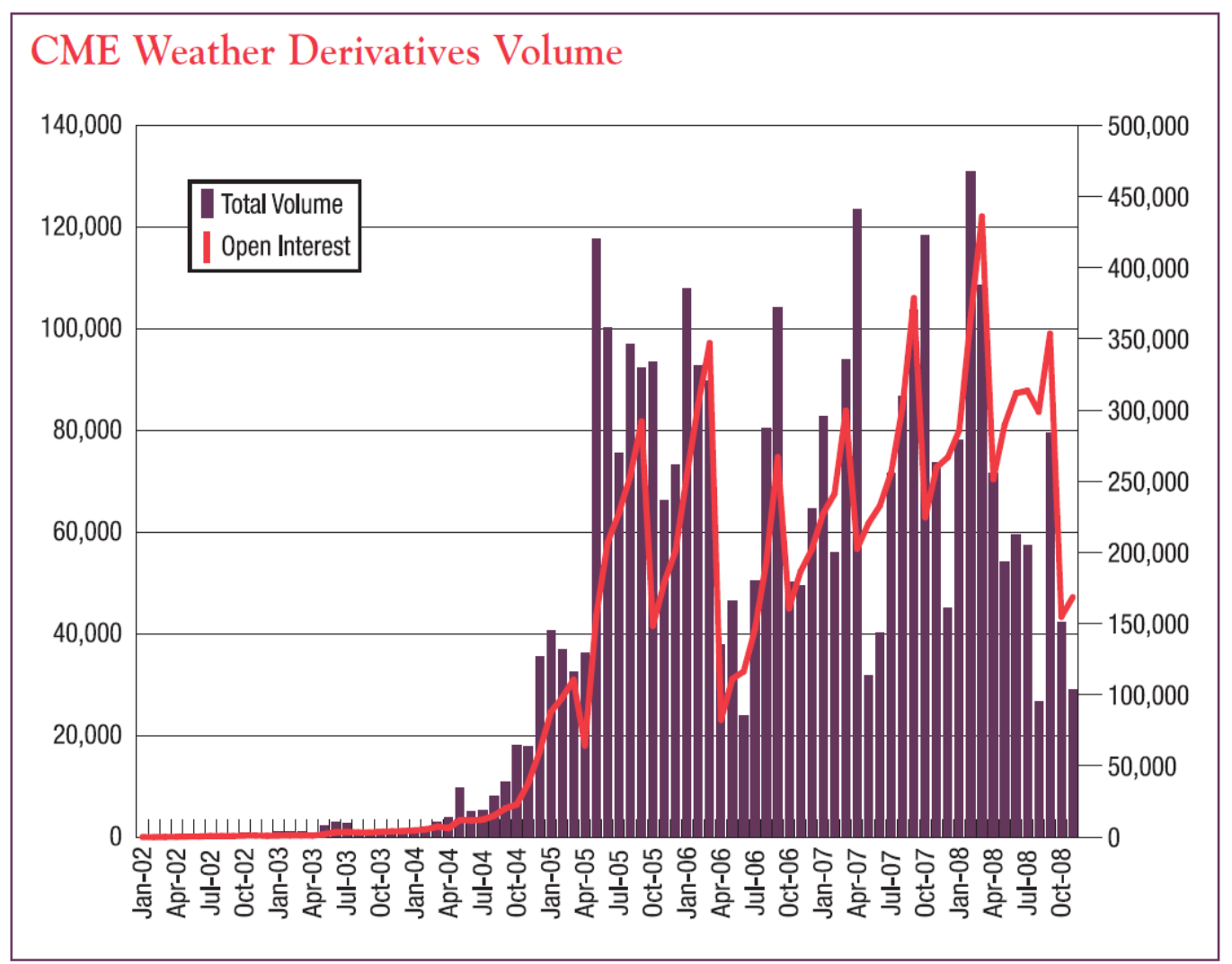

Figure 3-1.: CME Weather Derivatives Volume (Source: Futures Industry, January/February 2009). 


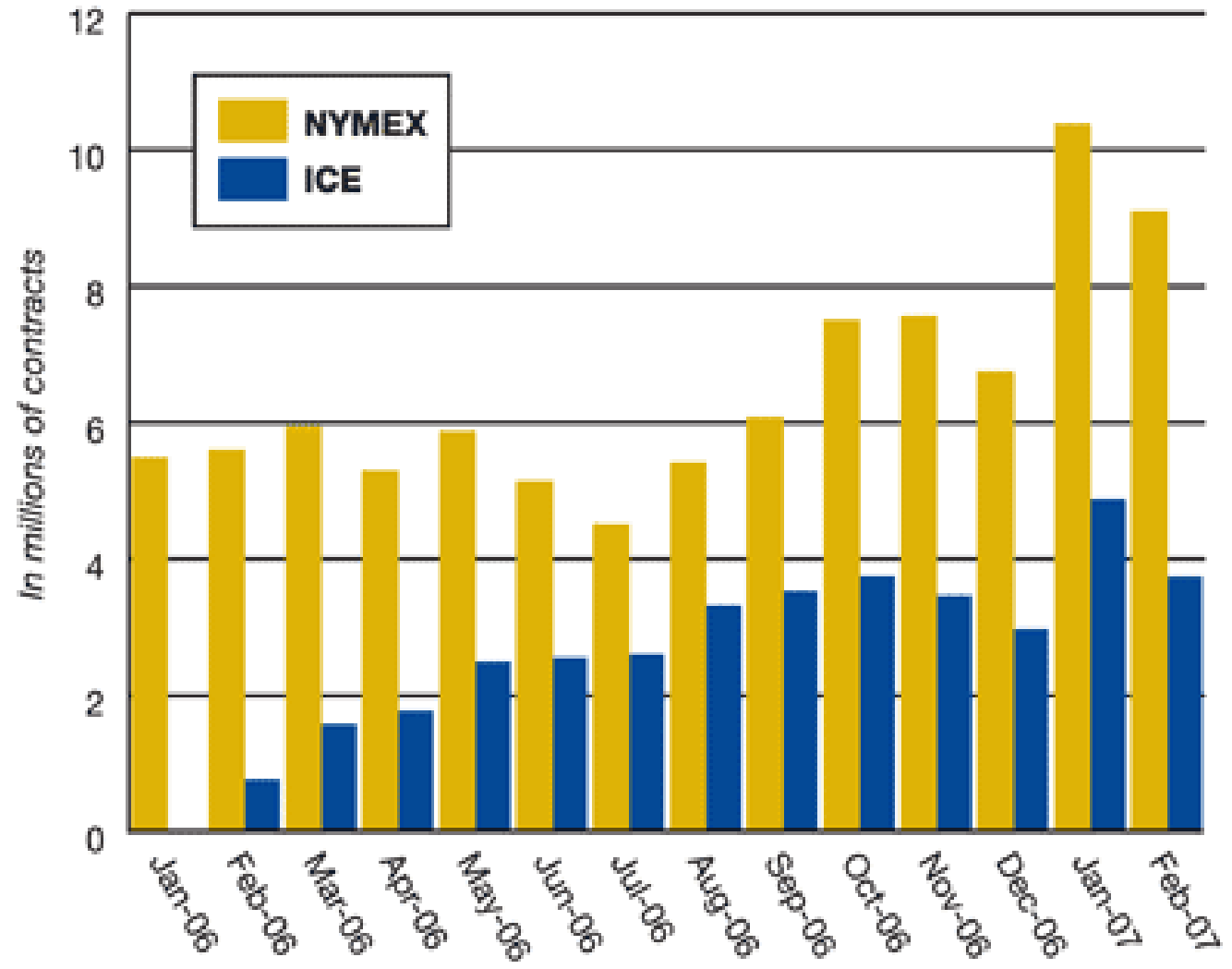

Figure 3-2.: WTI Crude Oil Futures traded at NYMEX and ICE (Source: Futures Industry, May/June2007). 


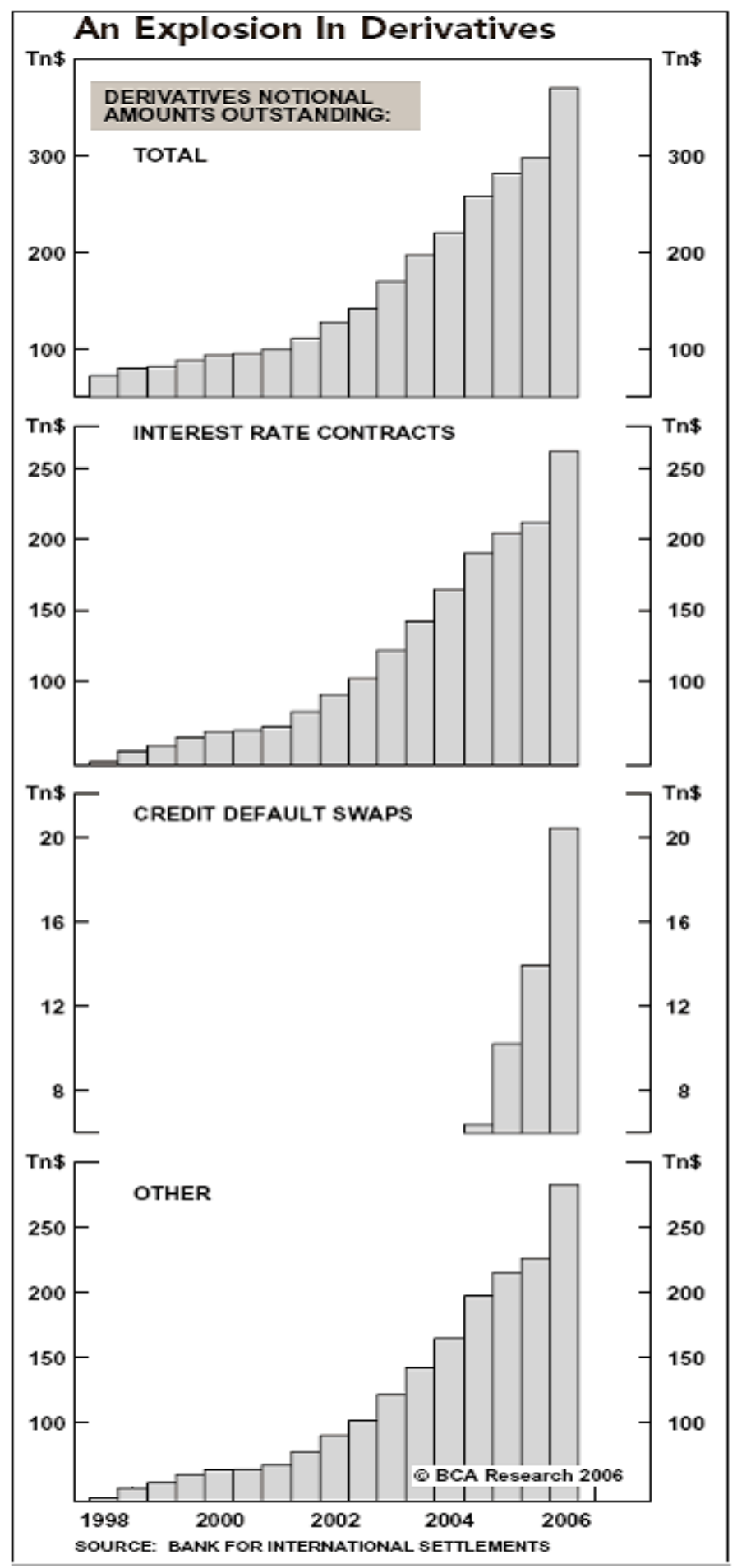

Figure 3-3.: Derivative Transaction Volume.

(Data Source: Bank for International Settlements, bis.org/) 


\subsection{Weather and Financial Risk}

Table 3-2 shows how different adverse weather types may impact various industries and thereby affecting financial revenue generated..

Table 3-2.: Links between Weather and Financial Risk

\begin{tabular}{|c|c|c|}
\hline Risk Holder & Weather Type & Risk \\
\hline Energy Industry & Temperature & $\begin{array}{l}\text { Lower sales during warm winters or cool } \\
\text { summers }\end{array}$ \\
\hline Energy Consumers & Temperature & $\begin{array}{l}\text { Higher heating/cooling costs during cold } \\
\text { winters and hot summers }\end{array}$ \\
\hline Beverage Producers & Temperature & Lower sales during cool summers \\
\hline $\begin{array}{r}\text { Building Material } \\
\text { Companies }\end{array}$ & Temperature/snowfall & $\begin{array}{l}\text { Lower sales during severe winters } \\
\text { (Construction sites shut down). }\end{array}$ \\
\hline Construction Companies & Temperature/snowfall & $\begin{array}{l}\text { Delays in meeting schedules during periods of } \\
\text { poor weather }\end{array}$ \\
\hline Ski Resorts & Snowfall & $\begin{array}{l}\text { Lower revenue during winters with below } \\
\text { average snowfall }\end{array}$ \\
\hline Agriculture & Temperature/Snowfall & $\begin{array}{c}\text { Significant crop losses due to extreme } \\
\text { temperature or rainfall }\end{array}$ \\
\hline Municipal Governments & Snowfall & $\begin{array}{c}\text { Higher snow removal costs during winters } \\
\text { with above-average snowfall }\end{array}$ \\
\hline Road Salt Companies & Snowfall & Lower revenues during low snowfall winters \\
\hline $\begin{array}{r}\text { Hydro-electric power } \\
\text { generation }\end{array}$ & Precipitation & Lower revenue during periods of drought \\
\hline
\end{tabular}

(climetrix.com) 


\subsection{Different elements of a Weather Contract/Derivative}

In this section, various elements/terms related to a weather related contract will be explained. These definitions have been compiled from the book on "Weather Derivative Valuation" by Jewson et al. (2005).

\section{Reference Weather Station}

All weather contracts are based on the actual observations of weather at one or more specific weather stations. Most transactions are based on a single station, although some contracts are based on a weighted combination of readings from multiple stations and others on the difference in observations at two stations.

\section{Index}

The underlying index of weather derivative defines the measure of weather which governs when and how payouts on the contract will occur. This is illustrated in more detail in the next section.

\section{Term}

All contracts have a start and an end date that contains the period over which the underlying index is calculated.

\section{Structure}

Weather derivatives are based on standard derivative structures such as puts, calls, swaps, collars, straddles and strangles. Key attributes of these structures are the strike (the value of the underlying index at which the contract starts to pay out), the tick size (the payout amount per unit increment in the index beyond the strike) and the limit (the maximum financial pay out of the contract). 


\section{Premium}

The buyer of a weather option pays a premium to the seller that is typically between 10 and $20 \%$

of the notional amount of the contract, however, this can vary significantly depending on the risk profile of the contract.

\subsection{Pay-off Diagram for Options}

Since options are non-linear instruments, it is a good idea to show their pay off diagrams. Pay-off diagrams can be used as a means of illustrating the risk/return profile of an option position. Payoff diagrams allow the interested party to visualize any trading strategy in a static form. The $\mathrm{x}$ axis (horizontal line) of a pay-off diagram represents the price of the underlying asset. The y-axis (vertical line) of a pay-off diagram represents the profit/loss on the option position. There are four basic pay-off diagrams for options: (1) a long call, (2) a long put, (3) a short call, and (4) a short put. These are discussed below.

\section{The Option Buyer}

\section{Long call}

The pay-off diagram below represents the effective pay-off of a long call position of an option at the time of the expiration date. It looks at the option from the point of view of the buyer (i.e. the investor). 


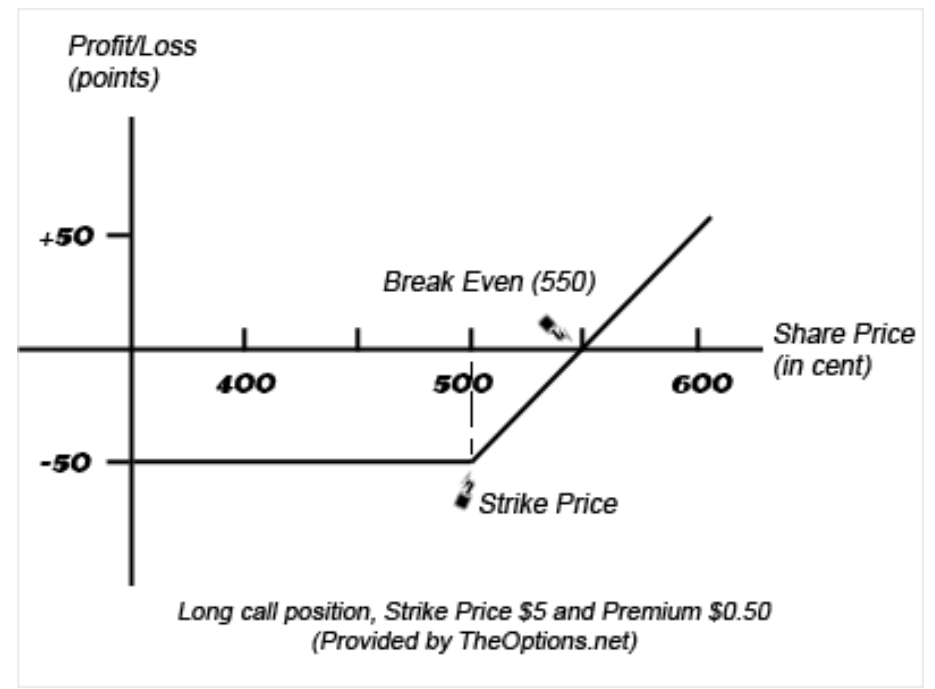

Figure 3-4: Pay-off diagram of long call for option buyer/holder.

(investopedia.com/theoptions.net)

If a trader believes the share of a company is on the rise, s/he can purchase a call option without buying the share. Assume s/he bought a call option (strike price is $\$ 5$ and premium is 50 cents). The breakeven point for this trade is $\$ 5.50$. At the expiration date, if the underlying share is trading at a point between $\$ 5$ and $\$ 5.50$, s/he will be able to recover a portion of the premium by exercising the option. If the share is above $\$ 5.50$ at this time, there will be a linear relationship between the share price and the profit. If the share price has moved up to $\$ 6$ by the option expiry date, the profit on the option will be $\$ 0.50$ (the different between the exercise price, which is $\$ 5$, and the current share price, less the option premium, which is $\$ 0.50$ )

\section{Long put}

The pay-off diagram below represents the effective pay-off of a long put position of an option at the time of the expiry date. It looks at the option from the point of view of buyer (again the investor). 


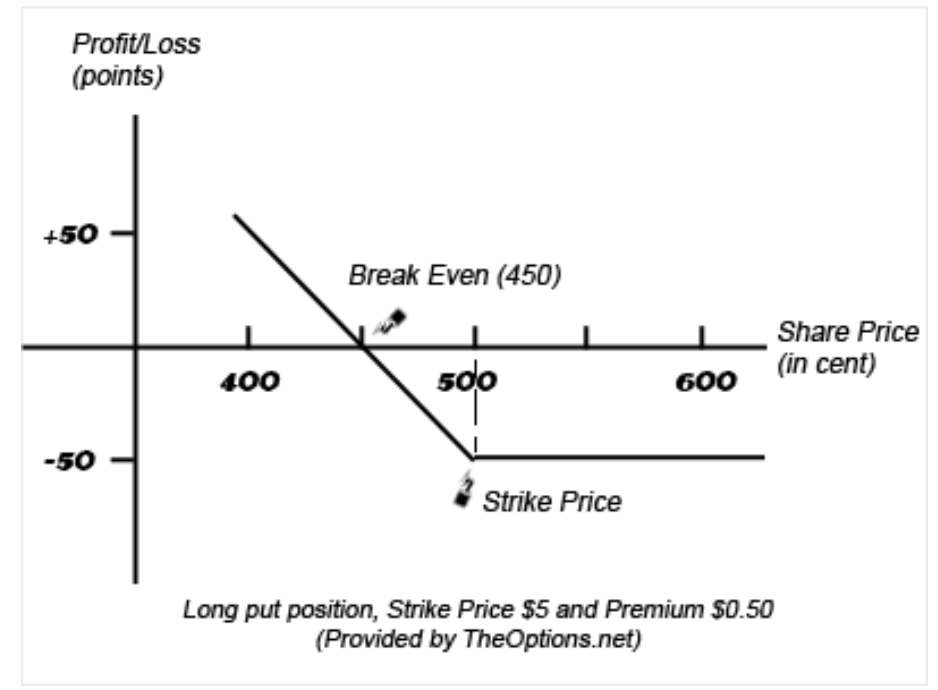

Figure 3-5.: Pay-off diagram of long put for option holder/buyer.

(investopedia.com/theoptions.net)

If a trader believes the share of a company is on the decline, s/he can make money by buying put options. Assume s/he bought a long option (strike price is $\$ 5$ and premium is 50 cents). Two weeks later, the share price falls to $\$ 4.50$. The put option now has intrinsic value of $\$ 0.50$. It will also have a time value, likely to be $\$ 0.30$. The option can now be sold in the marketplace for a premium value of $\$ 0.80$. The profit would be $\$ 0.30$. If at expiry date the underlying share is traded at or above $\$ 5$, there will be a loss of the full premium. The breakeven point for this option is $\$ 4.50$, which is equal to the strike price minus the premium. If the share is trading below $\$ 4.50$ by option expiry date, the option buyer would make a profit. There will be a linear relationship between the share price and the option profit.

The following pay-off is easier to understand by remembering "call up" and "put down", i.e., exercise call option if the market is up and exercise put option if the market is down. 


\section{The Option Seller}

\section{Short call}

The option seller has limited profit potential and potentially unlimited risk. The pay-off diagram below represents the effective pay-off of a short call position of an option at the time of the expiry date. It looks at the option from the point of view of seller (i.e. the writer of the option). The diagram below shows a call option with a premium of 50 cents. This position is the reverse of the buyer's. If the share price is below $\$ 5$ at the expiry date, the seller will earn the full premium 50 points, which is $\$ 0.50$. This is the most $\mathrm{s} /$ he can earn. The breakeven point for this option is the same as for the buyer, which is $\$ 5.50$. At this price, the premium earned will be exactly offset by the losses on the option position. If the share price moves above $\$ 5.50$, the option seller's loss moves in a linear relationship with the share price. At \$6, the option seller's loss will be $\$ 0.50$ (made up of $\$ 1$ loss on the option position less the premium earned of $\$ 0.50$ ).

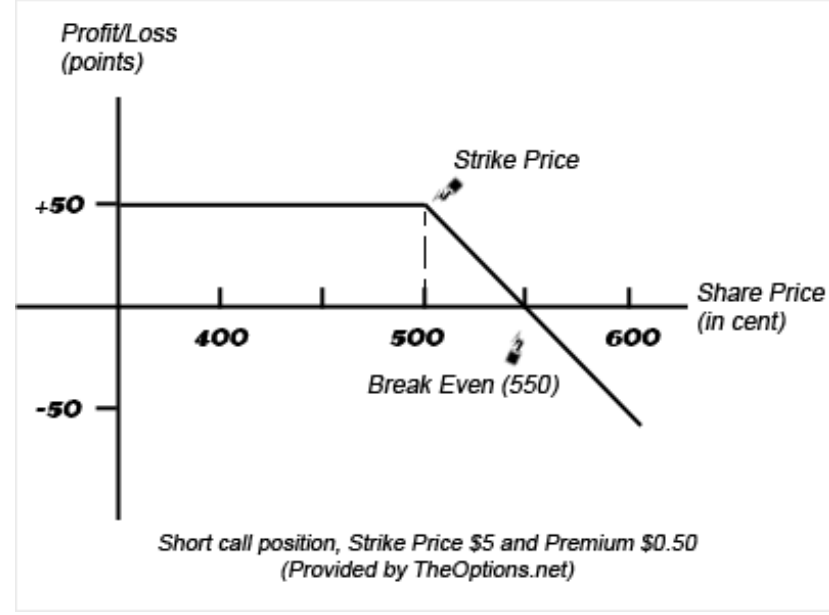

Figure 3-6.: Pay-off diagram for short call for option seller.

(investopedia.com/theoptions.net) 


\section{Short put}

The pay-off diagram below shows the effective pay-off of a short put position of an option at the time of the expiry date. It looks at the option from the point of view of the seller.

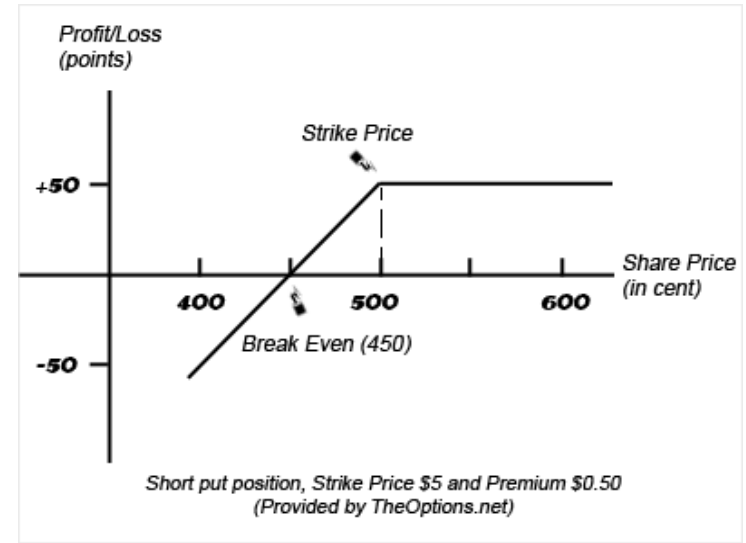

Figure 3-7.: Pay-off diagram of short put for option seller.

(investopedia.com/theoptions.net)

In this example, the seller will receive full premium if the share price is at or above the $\$ 5$ strike price. However if the share price drops, there is a linear relationship between the index and the loss made on this position. The breakeven point is $\$ 4.50$, where the profit is equal to the loss incurred.

\section{Pay-off Diagram of HDD Related Weather Derivative}

One common form of weather derivative as discussed in the subsequent section is a put option providing protection against a warm winter. Such a transaction might look like the following (climetrix.com).

Reference weather station: Chicago O'Hare International Airport (Weather Station: WBAN \#94846)

Underlying index: Heating Degree Days 
Term: Nov. 1 - Mar. 31.

Structure: Put option, Strike $=4850$ HDDs, Tick size $=\$ 5,000, \quad$ Limit $=\$ 1$ million, Premium \$150,000.

\section{Contract payoff diagram (net of premium)}

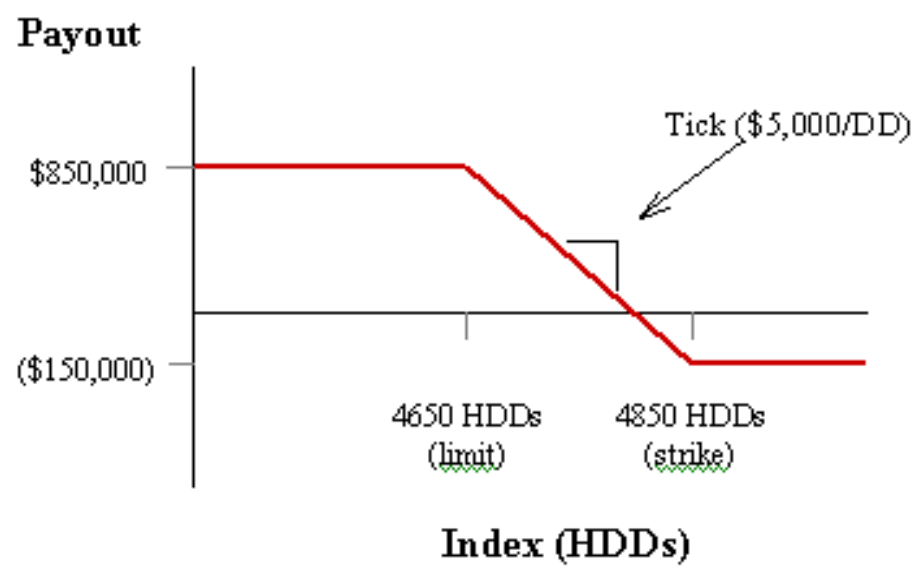

Figure 3-8.: Pay-off diagram of a HDD put (climetrix.com)

\subsection{Structure of Weather Derivatives}

In this section, structure of weather derivatives related to temperature and rainfall will be discussed. Currently, temperature related derivatives lead the market of weather derivatives.

\subsubsection{Temperature Based Call and Put Options}

Heating Degree Days (HDD) and Cooling Degree Days (CDD) contracts were responsible for over $85 \%$ of the weather derivative transactions made throughout 2005 (climetrix.com). The primary purpose of these contracts is to allow organizations to hedge against excessively high or low temperature distributions over a pre-specified period of time. Firstly, we define the average 
daily temperature as the arithmetic average of the maximum and minimum temperatures recorded in a $24 \mathrm{hr}$ period. In other words:

$\mathrm{T}_{\mathrm{i}}=\frac{\mathrm{T}_{\max }+\mathrm{T}_{\min }}{2}$

Naturally this average temperature is more reliable to model than either the maximum or minimum temperature are by themselves, however, information is thus lost and this measure would not provide a satisfactory hedge for those seeking protection from extreme daily temperatures. A HDD is simply the number of degrees the days average temperature was below

some reference level, $\overline{\mathrm{T}}$ generally set at $65^{\circ} \mathrm{F}$. Hence the HDDs for the month are given as:

$$
\mathrm{HDD}=\sum_{\text {month }} \max \left\{0,\left(\overline{\mathrm{T}}-\mathrm{T}_{\mathrm{i}}\right)\right\}
$$

The specific terminology derives from the fact that 'heating' is generally required for temperatures that are below the reference level (here set at $65^{\circ} \mathrm{F}$ ) thus requiring the expenditure of energy. The converse is also true for CDDs with the consumer's general requirement for 'cooling' energy above the reference temperature. From here an option contract can be set with a payoff and exercise price based on these measures. Hence a call option on the monthly HDD index would have a payoff of the form:

$$
\mathrm{V}_{\mathrm{t}}=\max \{0,(\mathrm{HDD}-\mathrm{k})\} \cdot \mathrm{tick}
$$

Where $\mathrm{k}$ is the exercise price of the option and the 'tick' provides the conversion from degrees to dollars (i.e. its units are $\$$ per degree) and generally ranges anywhere from $\$ 1,000$ to over $\$ 1,000,000$ per degree (speedwell.com).

A CDD contract is the reverse of the HDD in terms of its payoff. A CDD is just the number of degrees a particular day's average temperature was above some pre-determined reference level. As before the accumulated CDDs for a month are given as: 
$\mathrm{CDD}=\sum_{\text {month }} \max \left\{0,\left(\mathrm{~T}_{\mathrm{i}}-\overline{\mathrm{T}}\right)\right\}$

Other variables over which contracts are common include the monthly or daily average temperature (Asian type option ${ }^{1}$ ) as well as monthly and yearly cumulative temperatures. The maximum daily temperature provides a significant hedge to crop farmers against severe temperatures that can have a devastating impact on a harvest's quality and size. An example could be the production of wine whose yield and quality are highly dependent on temperatures during the two months prior to harvesting (wrma.org). If excessively high temperatures are encountered it can affect the physical vine growth as well as the quality of the grape produced including its flavor and odor. Hence a viticulturist could easily utilize a weather derivative over the maximum daily temperature experienced at a weather station that was in reasonably close proximity to the vineyard. This contract would compensate the farmer for reductions in yield and quality that result from the variations in the temperature.

\subsubsection{Rainfall Based Call and Put Options}

Rainfall contracts have received significantly less focus, in terms of both the literature and the amount of trading activity, when compared to temperature based derivatives. Primarily this was due to the fact that the weather derivatives market was largely created from the needs of energy and utility companies whose major exposure was to temperature related weather variations. Adding to this, rainfall has proven significantly more difficult to model accurately, particularly where geographically small areas are concerned. This is primarily due to the discrete nature of rainfall; two geographically close recording stations can produce widely different precipitation readings, something that is not encountered with temperature based statistics. These difficulties manifest as geographical 'basis' (discussed in later section) in rainfall derivative contracts in

\footnotetext{
${ }^{1}$ Please refer to Appendix G for an explanation of Asian/Bermudan Option types.
} 
that the situation of the risk must be relatively close to the measuring station for an effective hedge to be possible. This makes it increasingly hard to find the two (minimum) willing counterparties that all derivative contracts require.

The payoff for weather options usually takes the form of:

pay off for put $=\left[\begin{array}{cc}0 & \text { if } \mathrm{x}>\text { strike } \\ \text { strike }-x & \text { if } \mathrm{x}<=\text { strike }\end{array}\right] * \lambda$

pay off for call $=\left[\begin{array}{cc}0 & \text { if } \mathrm{x}<\text { strike } \\ x-\text { strike } & \text { if } \mathrm{x}>=\text { strike }\end{array}\right] * \lambda(20)$

Where strike is a choice variable, $\mathrm{x}$ is the cumulative realized value of the underlying index

(i.e. rainfall) during the contract period and $\lambda$ is some predetermined dollar value per unit of the index.

\subsection{Other Possible Weather Derivative Structures (Combinations) ${ }^{2}$}

A particular derivative or option strategy can be structure either as a single contract (either a call or put) or as a combination of two option contracts which is known as combination. In this section, various other possible weather derivative structures that can be constructed by a combination of call and put will be discussed. These definitions have been compiled from the book on "Weather Derivative Valuation" by Jewson et al. (2005). It is to be kept in mind that these structures are pertinent to mostly over-the-counter (OTC) derivatives rather than exchange traded.

\section{Call and Put Options With a Maximum Payoff (CAP)}

In order to avoid excessive payouts on these contracts due to extreme weather, the options often come with a cap or maximum payoff:

\footnotetext{
${ }^{2}$ Please refer to Appendix $\mathrm{H}$ for the pay-off diagram w.r.t. stock CAP/Collar/Straddle/Strangle.
} 
Payoff Call: $\operatorname{Min}\left(\mathrm{p}(\$ / \mathrm{D}) * \operatorname{Max}\left\{0, X_{t \mid T}-\mathrm{K}\right\}, \mathrm{h}\right)$

Payoff Put: Min (p (\$/D)*Max $\left.\left\{\mathrm{K}-0, X_{t \mid T}\right\}, \mathrm{h}\right)$

Where $\mathrm{h}$ is the maximum payoff denominated in dollars, $\mathrm{D}$ is the tick size and $\mathrm{K}$ is the strike rate as set forth in the contract. These are in usually set in dollar nomination in the contract.

\section{Collars}

A long collar position consists of a combination of a long call and a short put, usually with different strikes but the same tick and limit (although different ticks and limits are also possible). Collars have the following payoff function:

$$
p(x)=\left[\begin{array}{cc}
-\mathrm{L}_{\$} & \text { if } \mathrm{x}<\mathrm{L}_{1} \\
\mathrm{D}\left(\mathrm{x}-\mathrm{K}_{1}\right) & \text { if } \mathrm{L}_{1} \leq \mathrm{x}<\mathrm{K}_{1} \\
0 & \text { if } \mathrm{K}_{1} \leq \mathrm{x}<\mathrm{K}_{2} \\
\mathrm{D}\left(\mathrm{x}-\mathrm{K}_{2}\right) & \text { if } \mathrm{K}_{2} \leq \mathrm{x}<\mathrm{L}_{2} \\
\mathrm{~L}_{\$} & \text { if } \mathrm{x}>\mathrm{L}_{2}
\end{array}\right.
$$

Where $\mathrm{L}_{\$}$ and $\mathrm{L}_{2}$ are related by $\mathrm{L}_{\$}=\mathrm{D}\left(\mathrm{L}_{2}-\mathrm{K}_{2}\right)$ and $\mathrm{L}_{\$} \& \mathrm{~L}_{1}$ are related by $\mathrm{L}_{\$}=\mathrm{D}\left(\mathrm{L}_{1}-\mathrm{K}_{1}\right)$. This can also be written as:

$\mathrm{p}(\mathrm{x})=\max \left(-\mathrm{L}_{\$}, \min \left(\mathrm{D}\left(\mathrm{x}-\mathrm{K}_{1}\right), \max \left(0, \min \left(\mathrm{D}\left(\mathrm{x}-\mathrm{K}_{2}\right), \mathrm{L}_{\$}\right)\right)\right)\right)$

or by combining the expressions for calls and puts given above.

A long collar position provides a hedge against high values of the index for values beyond a certain threshold. Collars, like swaps, are usually costless.

\section{Straddles}

A long straddle position consists of a long call and a long put with the same strike, tick and limit. Straddles have the pay-off function: 


$$
p(\mathrm{x})=\left[\begin{array}{cc}
\mathrm{L}_{\$} & \text { if } \mathrm{x}<\mathrm{L}_{1} \\
\mathrm{D}(\mathrm{K}-\mathrm{x}) & \text { if } \mathrm{L}_{1} \leq \mathrm{x}<\mathrm{K}_{1} \\
\mathrm{D}(\mathrm{x}-\mathrm{K}) & \text { if } \mathrm{K} \leq \mathrm{x}<\mathrm{L}_{2} \\
\mathrm{~L}_{\$} & \text { if } \mathrm{x}>\mathrm{L}_{2}
\end{array}\right.
$$

Where $\mathrm{L}_{\$}$ and $\mathrm{L}_{1}$ are related by $\mathrm{L}_{\$}=\mathrm{D}\left(\mathrm{K}-\mathrm{L}_{1}\right)$ and $\mathrm{L}_{\$} \& \mathrm{~L}_{2}$ are related by

$\mathrm{L}_{\$}=\mathrm{D}\left(\mathrm{L}_{2}-\mathrm{K}\right)$. This can also be written as:

$\mathrm{p}(\mathrm{x})=\min \left(\mathrm{L}_{\$}, \max \left(\mathrm{D}(\mathrm{K}-\mathrm{x}), \min \left(\mathrm{D}(\mathrm{x}-\mathrm{K}), \mathrm{L}_{\S}\right)\right)\right)$

A long straddle position hedges against both high and low values of the index and is hence typically rather inexpensive in terms of premium since the buyer receives a pay-off for all values of the index except $\mathrm{x}=\mathrm{K}$.

\section{Strangles}

A long strangle consists of a long call and a long put but with different strikes (unlike a straddle, where strikes are the same). The strike for the put is usually lower than that for the call.

Strangles has the pay-off function:

$$
p(x)=\left[\begin{array}{cc}
\mathrm{L}_{\$} & \text { if } \mathrm{x}<\mathrm{L}_{1} \\
\mathrm{D}\left(\mathrm{K}_{1}-\mathrm{x}\right) & \text { if } \mathrm{L}_{1} \leq \mathrm{x}<\mathrm{K}_{1} \\
0 & \text { if } \mathrm{K}_{1} \leq \mathrm{x}<\mathrm{K}_{2} \\
\mathrm{D}\left(\mathrm{x}-\mathrm{K}_{2}\right) & \text { if } \mathrm{K}_{2} \leq \mathrm{x}<\mathrm{L}_{2} \\
\mathrm{~L}_{\$} & \text { if } \mathrm{x}>\mathrm{L}_{2}
\end{array}\right.
$$

Where $L_{\$}$ and $L_{2}$ are related by $L_{\$}=D\left(L_{2}-K_{2}\right)$ and $L_{\$}, L_{1}$ are related by

$L_{\$}=D\left(L_{1}-K_{1}\right)$. This can also be written as

$$
\mathrm{p}(\mathrm{x})=\min \left(\mathrm{L}_{\$}, \max \left(\mathrm{D}\left(\mathrm{K}_{1}-\mathrm{x}\right), \max \left(0, \min \left(\mathrm{D}(\mathrm{x}-\mathrm{L}), \mathrm{L}_{\$}\right)\right)\right)\right)
$$


A long strangle position hedges against both high and low values of the index in a similar way to a straddle, but pays out only when the index moves a certain distance. They are thus typically a little cheaper than straddles.

\section{Weather Swaps}

The pay-off, $\mathrm{p}$, from a long swap contract is given by:

$p(x)=\left[\begin{array}{cc}-L_{\$} & \text { if } x<L_{1} \\ D(x-K) & \text { if } L_{1} \leq x \leq L_{2} \\ L_{\$} & \text { if } x>L_{2}\end{array}\right.$

Where $\mathrm{x}$ is the index, $\mathrm{D}$ is the tick, $\mathrm{K}$ is the strike, $L_{\$}$ is the limit expressed in currency terms and

$L_{1}$ and $L_{2}$ are the upper and lower limits expressed in units of the index. $L_{\$}$ and $L_{1}$ are related by $L_{\$}=D\left(K-L_{1}\right)$ and $L_{\$} \& L_{2}$ are related by $L_{\$}=D\left(L_{2}-K\right)$. This payoff can also be written as: $p(x)=\max \left(-L_{\$}, \min \left(D(x-K), L_{\$}\right)\right)$ Or $p(x)=\min \left(L_{\S}, \max \left(D(x-K),-L_{\$}\right)\right)$

The upper and lower limits (in monetary terms) are set to be equal and opposite. More generally, it would be possible to structure a swap contract (and also all the other contract types) with limits of different sizes on the two sides, although this is very unusual. It is also possible to structure swaps without financial limits; the pay-off is then a linear function of the index, given by $p(x)=D(x-K)$. This is called a linear swap. Even for swaps with limits, the limits are usually set at rather extreme values. OTC contracts are usually traded with limits while the CME contracts do not have limits. Most swaps are costless, i.e. there is no premium, and the profit or loss from a swap is equal to the pay-off. When the contract is set up the two counterparties simply exchange a contract agreeing to pay each other according to the weather at some point in the future. In this sense, a swap is like a spread bet on the future weather. A long swap contract has the economic function of insuring against high values of the index. 


\section{Binaries}

A long binary option has a pay-off function of the form

$$
p(x)=\left[\begin{array}{cc}
0 & \text { if } x<K \\
L_{\$} & \text { if } x \geq K
\end{array}\right.
$$

A long binary option can be considered to be a special case of a call option. In this case, in which the index $\mathrm{x}$ is considered to be continuous; it is a call option with infinite tick. In the case in which the index $\mathrm{x}$ is a discrete number to certain values with spacing $\square x$ the tick is equal to $\frac{\mathrm{L}_{\$}}{\mathrm{Vx}}$.

\subsection{Other Exotic Weather Derivatives}

Examples of exotic weather derivatives other than based on temperature or rainfall include those that depend on snowfall, wind speed, etc. Among these categories, snowfall derivatives have shown better promise as they have been introduced at CME December 2009 for five US cities. Although the number of snowfall contracts is still in the hundreds, leading industry experts are very optimistic about them. The idea behind a snowfall derivative is similar to what has already been discussed, i.e., a business which is directly affected by snowfall can use the market to transfer the risk of uncertainty in weather. Here is a brief hypothetical case study of a snowfall derivative that might work well for a snow removal company:

The winter of 2009-2010 has been one of the harshest in West Virginia. The University had to be shut down twice during January-February 2010. The city of Morgantown spent a few million dollars in snow removal activities in winter 2010. From this experience comes this hypothetical case study utilizing snowfall derivative which has already been used in a few places, including Chicago. This case study is concerned with a snow removal company. For these types of parties, uncertainty in winter weather, particularly snowfall levels, presents an inherent risk. In order to do their job, these companies must have all their supplies and labor on 
hand to effectively respond when snow hits. Most of these firms set up contracts in the spring and summer in which they commit to removing snow the following winter. These companies need to bear fixed costs with getting the contract, which includes staff, trucks, salt, gas, etc. If snowfall is low, they will not meet those costs or the business volume will not be high enough to cover for all these costs. Some supplies can be stored but others cannot and the company is forced to absorb the cost, hurting the snow-removal company's bottom-line. For example, salt gets wet and evaporates. On the opposite end of the spectrum, if snow levels are much higher than expected, extra staff is hired, overtime is paid and additional supplies are purchased. All of these factors increase overhead, which is generally higher once winter begins, leading to declining revenue margins.

The first snowfall contracts were listed in 2006, futures contracts based on snowfall at Boston Logan International Airport and New York Central Park. CME now offers six snowfall contracts based on weather data from six locations, in five U.S. cities: Boston, Chicago, Detroit, Minneapolis and two in New York. This range includes futures and options, but so far only two of the contracts have traded: the seasonal and monthly binary options (discussed in section 3.9). A snowfall binary option allows end-users to protect themselves from extra expenses if snowfall is outside of expectations. If a snow removal company has supplies to plow up to a certain amount of snow it can buy a binary options contract with a strike point at that level of snowfall. If snowfall is above the strike point, the company can "call" the option and is paid a fixed-dollar amount for whatever the contract specifies. That money can be used to cover extra expenses in the case of too much snow. If snowfall is below the strike price, the option simply expires worthless. On the opposite side, if a company chose to use "pay per event" contracts in which they are paid for each time their services are used, they could hedge against the possibility of too 
little snow by selling binary options. If snowfall is low, the premium they received from selling the options supplements the bottom line. If snow is piling up, they pay out on the option but have increased revenue with which to do so. In essence, these contracts function as an insurance policy against potential losses if snow levels inhibit business or profit margins. So this is an extra risk management strategy for the companies affected by snowfall (too much or too little) in addition to usual snowfall related insurance policy. There are players in the market who can underwrite the snowfall related risk and provide an insurance coverage. However, many market practitioners rely on weather derivatives traded on CME rather than an insurance company simply because CME insures guaranteed pay out by working as a clearing house. During recent turmoil, many insurance companies have defaulted on indemnity payments. Also, the capital market may provide better transparency and more choices compared to traditional insurance players.

\subsection{Geographic Basis Risk}

Basis risk is defined as the risk that offsetting investments in a hedging strategy will not experience price changes in entirely opposite directions from each other. This imperfect correlation between the two investments creates the potential for excess gains or losses in a hedging strategy, thus adding risk to the position. Geographic basis risk is very unique to weather derivatives as weather is a very spatial phenomenon; hence the underlying index needs to be correctly tied to the local weather station data.

\subsection{Precipitation/Rainfall Modeling}

A review of the literature reveals three types of rainfall models that have been used in pricing rainfall derivatives. They are: generalized linear models or GLMs, hidden Markov models and Non parametric models. GLM models were introduced by Coe and Stern $(1982,1984)$ to model a daily time series of rainfall measurement at a single site. GLMs are the simplest stochastic 
weather models and have been used widely due to their simplicity. These are basically parametric models with the outcome variable as the daily rainfall which is modeled with explanatory variables of sine/cosine functions of time for seasonality. They also sometimes incorporate other covariates like climatological variables and regional forecasts. Single site models can be enhanced to model multiple sites using multivariate time series methods. In these models, correlations across space are modeled together with rainfall outcomes at unobserved locations. The marginal distribution of daily rainfall data has a point mass at zero (dry days), so these models often comprise two parts: 1. A model for dependent binary data-such as a two-state Markov chain (as has been used in this study) model to model the occurrence of rainfall on a given day conditional on whether the previous day was dry or wet. 2. For wet days, a rightskewed distribution such as exponential, gamma or mixed exponential distributions are used to model the magnitude of rainfall. Most GLMs are fit using maximum-likelihood methods (Jewson, 2002).

Hidden Markov Chain or HMM models differ from GLMs in that they model autocorrelation and spatial correlation through some number, $\mathrm{K}$, of discrete, unobserved hidden states (Jewson, 2002). These hidden states often correspond to different "weather states" that result in different patterns of rainfall. These hidden states change through time according to a first-order Markov chain and the outcomes are modeled as independent draws from distributions conditional on their corresponding hidden states.

Nonparametric models are attractive due to their freedom from parametric distribution assumptions. Hence these models may be best suited to model certain features of the rainfall process better than GLMs or HMM. The key feature of these models is a resampling algorithm, in which daily time series of rainfall are simulated by resampling the observed data in a way that 
accounts for the autocorrelation of observed rainfall (Wilks, 1989). This also takes care of the relationships between rainfall and other weather variables.

\section{Local Correlation of Weather Stations of WV}

Temperatures diffuse quickly to produce homogenous areas with nearly uniform levels of the desired statistics. Rainfall, on the other hand, is discrete by its very nature and this provides difficulties when attempting to produce reliable models of its variability. This local variability manifests already discussed geographic basis risk. This is a huge limitation when considering the use of derivatives to hedge rainfall/precipitation exposure and is one of the primary reasons that these types of weather derivatives are slow to be taken up compared with temperature derivatives. As a remedy, the derivative contract can be based on the average of several nearby weather stations in order to reduce much of the spatial basis risk that is inherent in precipitation derivatives. Hence two weather stations in close proximity to each other often record vastly different rainfall measures on the same day. The local rainfall correlations between Monongalia/Hartfield and Martinsburg/Berkeley weather stations of WV are reported in section 3.5 along with other results.

\section{Linear Trend}

It is important to check for long term trends which are usually found in temperature data. A regression was performed on the yearly cumulative rainfall of Monongalia/Hartfield Airport to check if any significant trend can be established. The result is included again in the results section.

\section{Transition Probability and Seasonality}

Following Cao (2004), let $X_{t}$ be a binary variable that takes a value of 1 if it rains on day $t$ and 0

otherwise. $X_{t}$ is an nth order Markov chain if $X_{t}$ is independent of $X_{t-k}$ for all $k>n$. Let us keep 
only the first order Markov Chain i.e. $n=1$. Let $\mathrm{p}$ be the probability that day $\mathrm{t}$ is wet, then for the first order Markov chain we have,

$$
p_{t}=p_{t-1} q_{11}+\left(1-p_{t-1}\right) q_{01}(32)
$$

Where $\mathrm{q}_{11}$ and $\mathrm{q}_{01}$ are the one-step transition probabilities.

In layman's terms this is equivalent to asking:

What is the probability of wet today, conditional on a dry yesterday or a wet yesterday?

If today is wet, then what is the expected rainfall/precipitation?

Given two states, Dry (D) or Wet (W), there could be four possible one stage transition probabilities:

$$
\begin{aligned}
& \text { Dry (D) } \mid \mathrm{t}-1 \longrightarrow \text { Wet }(\mathrm{W}) \mid \mathrm{t} \quad(\mathrm{a}) \\
& \text { Wet }(\mathrm{W})|\mathrm{t}-1 \longrightarrow \operatorname{Dry}(\mathrm{D})| \mathrm{t} \\
& \text { Dry (D) }|\mathrm{t}-1 \rightarrow \operatorname{Dry}(\mathrm{D})| \mathrm{t} \quad \text { (c) } \\
& \text { Wet }(\mathrm{W})|\mathrm{t}-1 \rightarrow \operatorname{Wet}(\mathrm{W})| \mathrm{t} \quad(\mathrm{d})
\end{aligned}
$$

We are interested only in (a) which is denoted by $q_{01}$ and (d) which is denoted by $q_{11}$. Conditional on $\mathrm{X}_{\mathrm{t}}=1$ (i.e. a wet day), the amount of rainfall $Y_{t}$ follows a gamma distribution, and the probability density function is:

$$
f(x, \alpha, \beta)=\left(\frac{x}{\beta}\right)^{\alpha-1}\left[\frac{\exp \left(\frac{-x}{\beta}\right)}{\beta \tau(\alpha)}\right], x>0 ; \alpha, \beta>0,
$$

Where $\alpha$ is the shape parameter and $\beta$ is the scale parameter, $\mathrm{x}$ is the random variable which is cumulative precipitation in this case. Scale and shape parameters can be estimated using MLE (Greene, 2007). 
Seasonality can also be built into the framework. We can allow the one-step transition probability $q_{11}$ to vary with the time of the year as follows:

$$
\mathrm{q}_{11}=\mathrm{c}_{0}+\sum_{\mathrm{i}=1}^{\mathrm{m}}\left[\mathrm{c}_{1 \mathrm{i}} \sin \left(\frac{2 \pi \mathrm{i}}{365} \mathrm{t}\right)+\mathrm{c}_{2 \mathrm{i}} \cos \left(\frac{2 \pi \mathrm{i}}{365} \mathrm{t}\right)\right]
$$

Where $t$ is time and $\mathrm{m}$ is an integer to be set by the user, c's are the parameters to be estimated. The mean conditional precipitation for day $\mathrm{t}$ can be modeled as:

$$
\overline{\mathrm{Y}}_{\mathrm{t}}=\mathrm{d}_{0}+\sum_{\mathrm{i}=1}^{\mathrm{m}}\left[\mathrm{d}_{1 \mathrm{i}} \sin \left(\frac{2 \pi \mathrm{i}}{365} \mathrm{t}\right)+\mathrm{d}_{2 \mathrm{i}} \cos \left(\frac{2 \pi \mathrm{i}}{365} \mathrm{t}\right)\right]
$$

Where $\mathrm{m}$ is same as above and d's are the parameters to be estimated.

If the transition probabilities (i.e. the transition from either a dry or wet day at (t-1) or previous day to a wet day at t or present day) are constant, then the maximum likelihood estimate for $q_{01}($ $q_{11}$ ) is the ratio of The total number of raining days where the previous day is dry (wet) and The number of dry (wet) days.

To estimate the seasonal transition probabilities as defined in equation (34), the following regression model can be estimated for those days where it rained the previous day:

$$
\mathrm{I}_{\mathrm{t}}(1)=\overline{\mathrm{c}}_{0}+\sum_{\mathrm{i}=1}^{\mathrm{m}}\left[\hat{\mathrm{c}}_{1 \mathrm{i}} \sin \left(\frac{2 \pi \mathrm{i}}{365} \mathrm{t}\right)+\hat{\mathrm{c}}_{2 \mathrm{i}} \cos \left(\frac{2 \pi \mathrm{i}}{365} \mathrm{t}\right)\right]+\varepsilon_{\mathrm{t}}
$$

Where $\mathrm{I}_{\mathrm{t}}(1)=1$ if day $\mathrm{t}$ rains and 0 otherwise. $\varepsilon_{t}$ is the regression error term.

Use of a gamma distribution in modeling cumulative rainfall is popular in previous Rainfall derivative modeling work (Martin et al, 2001). Cao, 2004 has shown use of both parametric and non parametric (kernel density) methods to model the cumulative rainfall. I have used both the gamma and exponential distribution to model the rainfall. SAS/ETS has been used for the estimation purposes. It generates Kolmogorov-Smirnoff and Anderson-Derling test statistics 
which can be used to compare the goodness-of-fit of both parametric and non parametric assumptions.

\subsection{Simulation}

Once the parameters have been estimated for both frequency and magnitude processes, it is possible to simulate precipitation values and compare them with historical values. A simulation exercise has been carried out for Monongalia/Hartfield weather station historical data which is reported in section 3.5 .

\subsection{The Effects of El Nino}

The main source of seasonal predictability is a phenomenon known as El Nino Southern Oscillation. The 'El Nino' part of ENSO is a fluctuation in the surface temperatures of the eastern half of the equatorial Pacific Ocean, while the 'Southern Oscillation' part is a shift in the wind and pressure patterns over the whole of the Pacific (Jewsen, 2007). In fact, the two phenomena are intimately linked through a cycle of cause and effect, and they are usually discussed as part of the same phenomenon: hence the name ENSO. The changes in the atmosphere associated with El Nino cause changes in the weather around large parts of the globe. In particular, El Nino has very strong effects in other parts of the tropics: this is because atmospheric signals travel most easily along the equator. Certain parts of South America and Australia experience very strong El Nino effects, while parts of Africa and the Indian Ocean also feel some effect. The impact of El Nino on the extratropics is weaker. Among these weaker signals, the strongest are those in North America. Many studies (Jewson, 2002) have been carried out to quantify such effects, although precise quantification is very difficult for a number of reasons, such as (a) every El Nino is slightly different, (b) there have only been a small number of El Nino events during the last forty years, and (c) the effects are not necessarily simple in structure. However, two basic features of the response to El Nino over the United 
States during the winter have been observed quite frequently. They are: s warming over most of the northern United States, and increased rainfall in the southern United States and coastal California. El Nino effects have not been considered in this study, since West Virginia is quite free from its effects.

\subsection{Rainfall/Precipitation Data, Estimation and Results}

NOAA data from four weather stations in West Virginia were obtained. The weather stations are: Hartfield airport of Monongalia County, Martinsburg of Berkeley County, Monroe County and Randolph County. Each weather station contains about ten thousand observations of the past 30 years of daily data (1971-2000).

\section{Precipitation Trend/Seasonality}

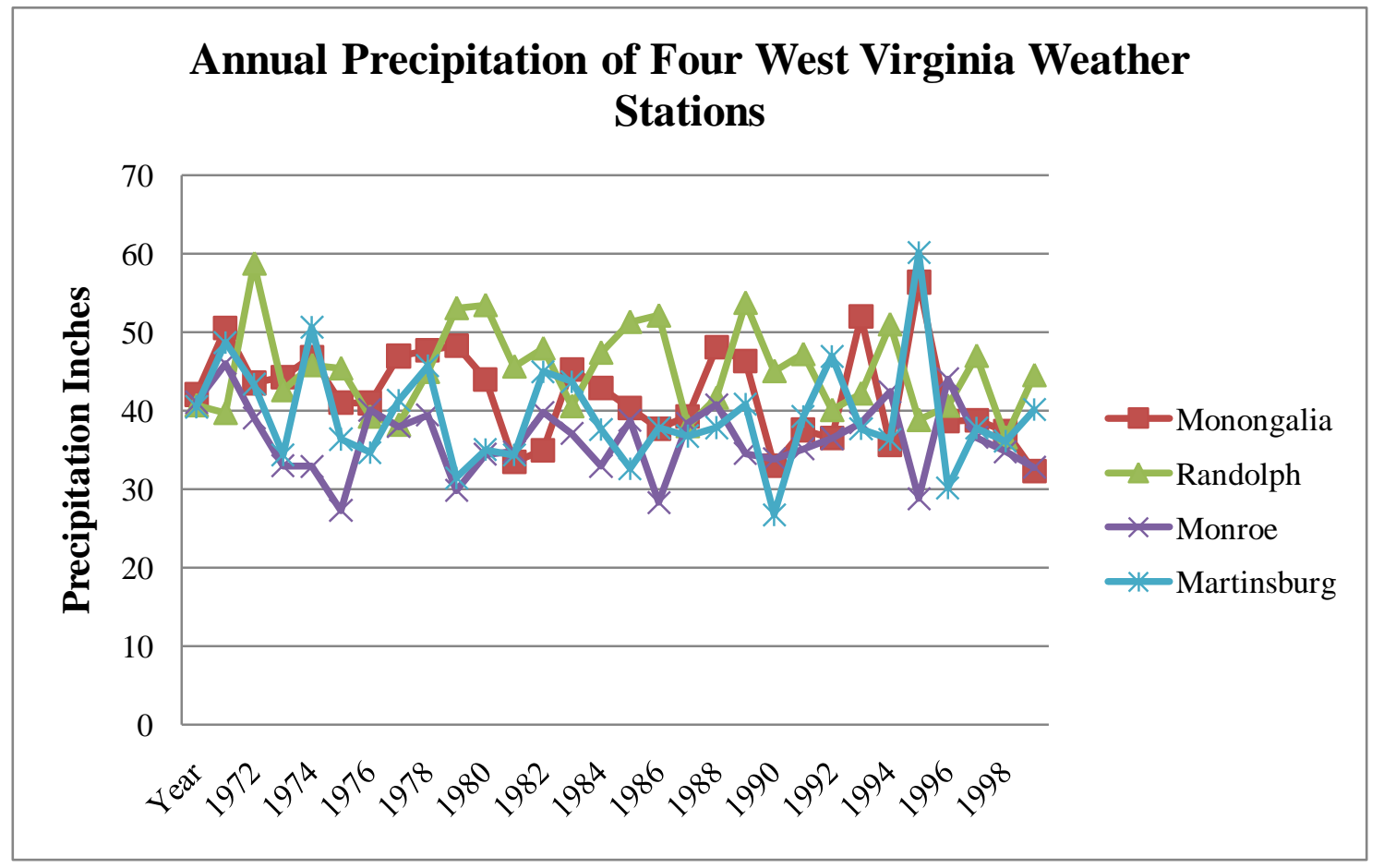

Figure 3-9.: Precipitation Trend of Annual Precipitation of Four West Virginia Weather Stations over 1971-2000. 


\section{Local Trend}

Monthly Precipitation Trend-Monongalia/Hartfield vs. Martinsburg

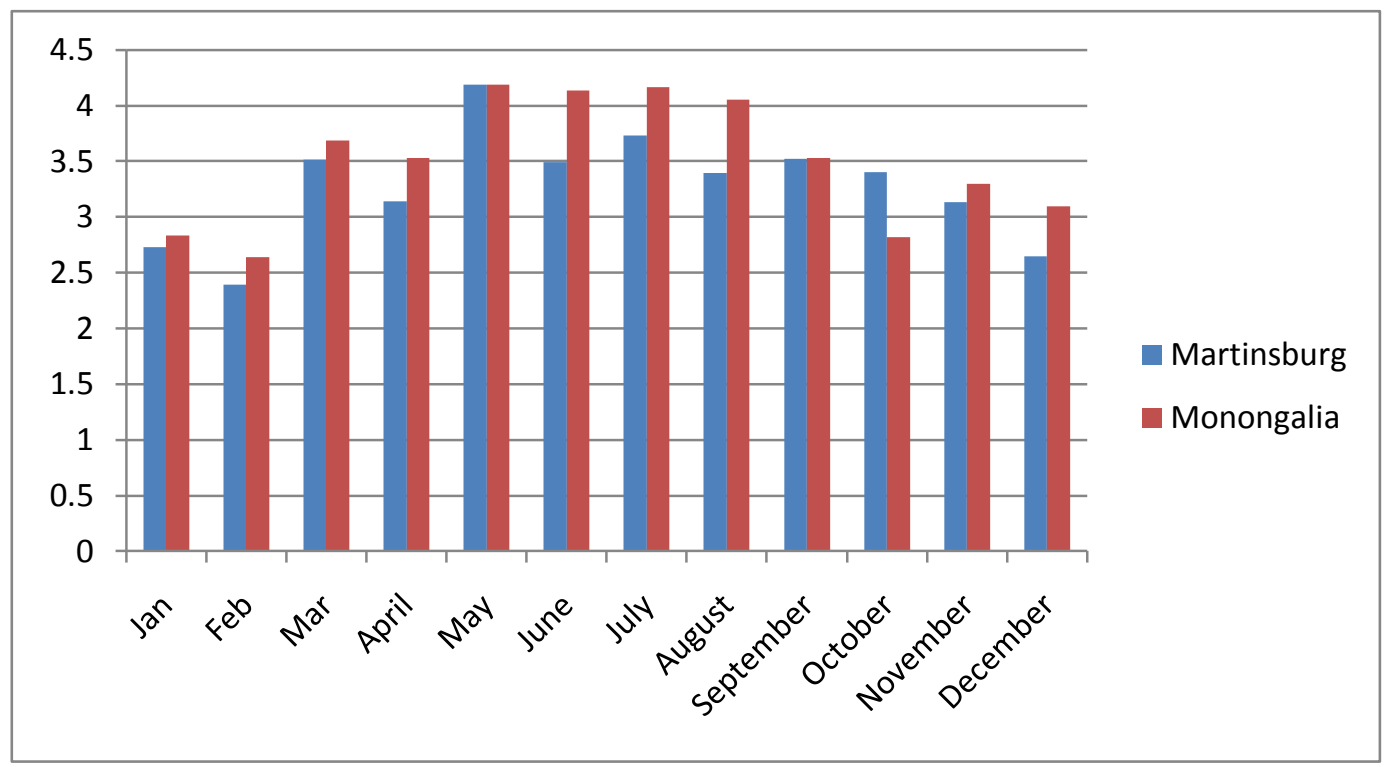

Figure 3-10.: Monthly Precipitation Trend-Monongalia/Hartfield vs. Martinsburg (19712000).

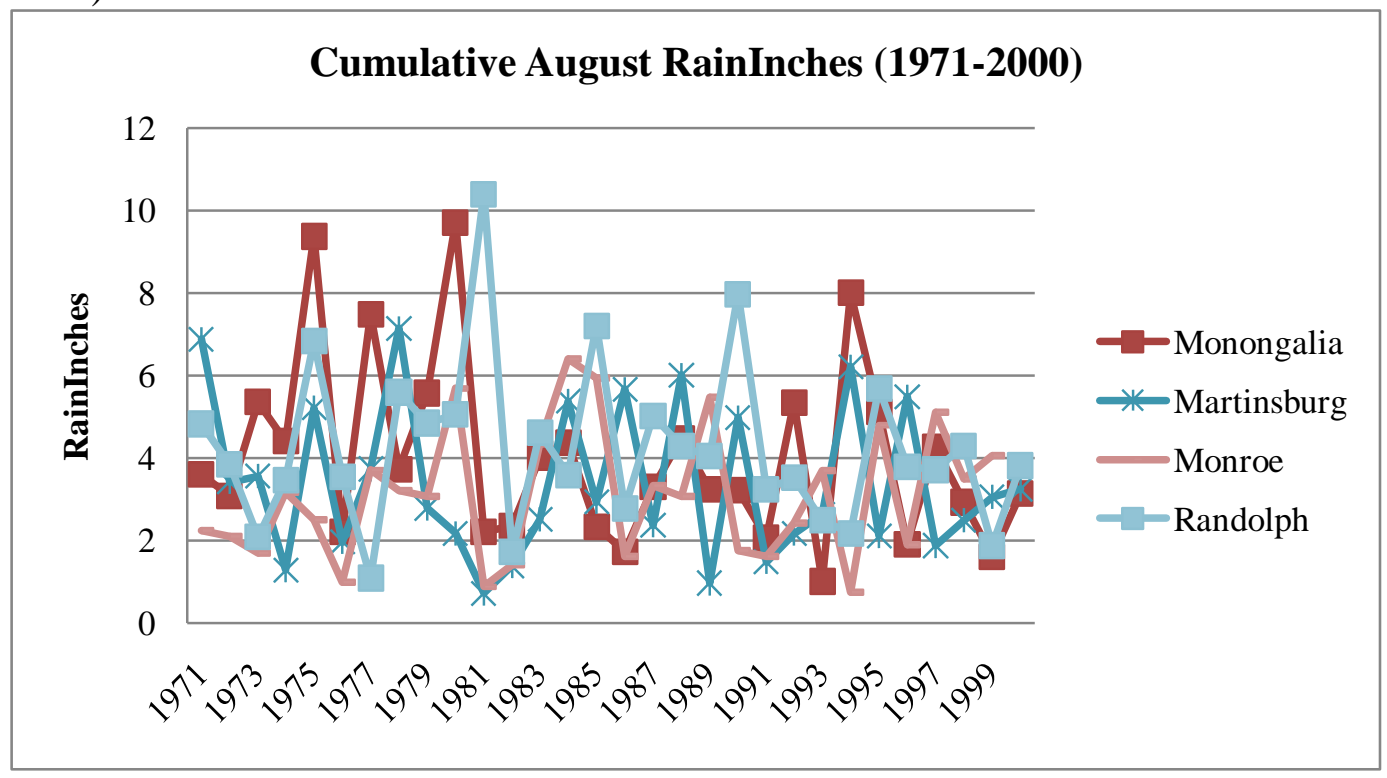

Figure 3-11.: Cumulative August Rain Inches (1971-2000) of Four West Virginia Weather Stations. 


\section{Linear Trends}

\section{Annual Cumulative Rainfall-Monongalia/Hartfield}

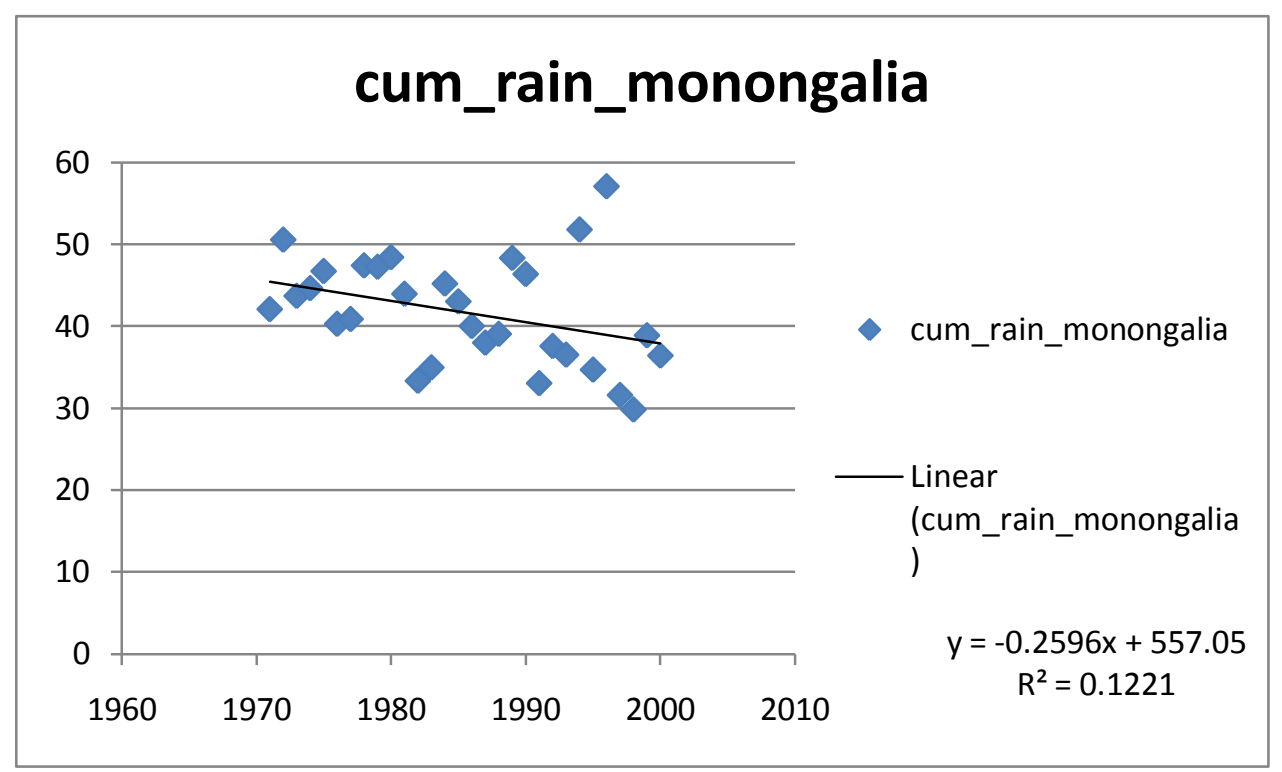

Figure 3-12.: Annual Cumulative Precipitation-Monongalia/Hartfield

From the R-squared value, it is evident that there is no linear trend present.

Transition Probability and Seasonality

Monongalia

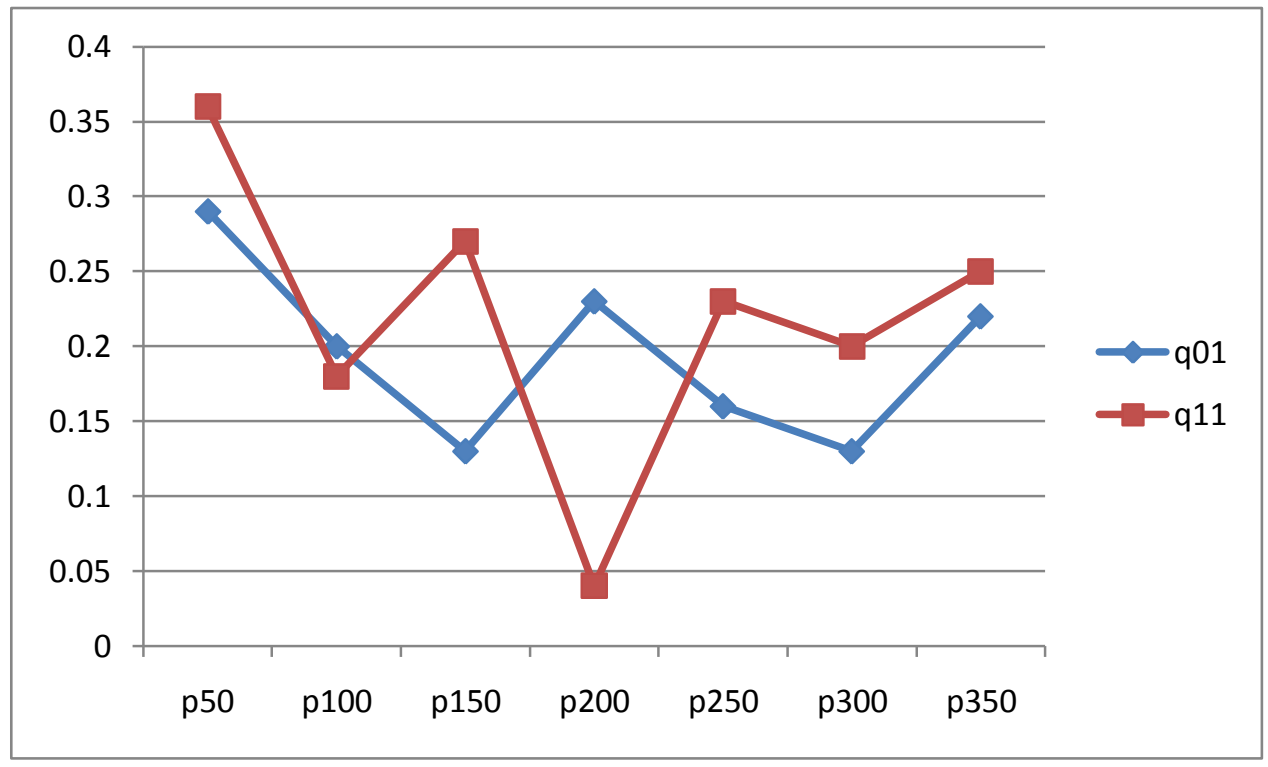

Figure 3-13: Transition Probability (q01/q11) for Monongalia 


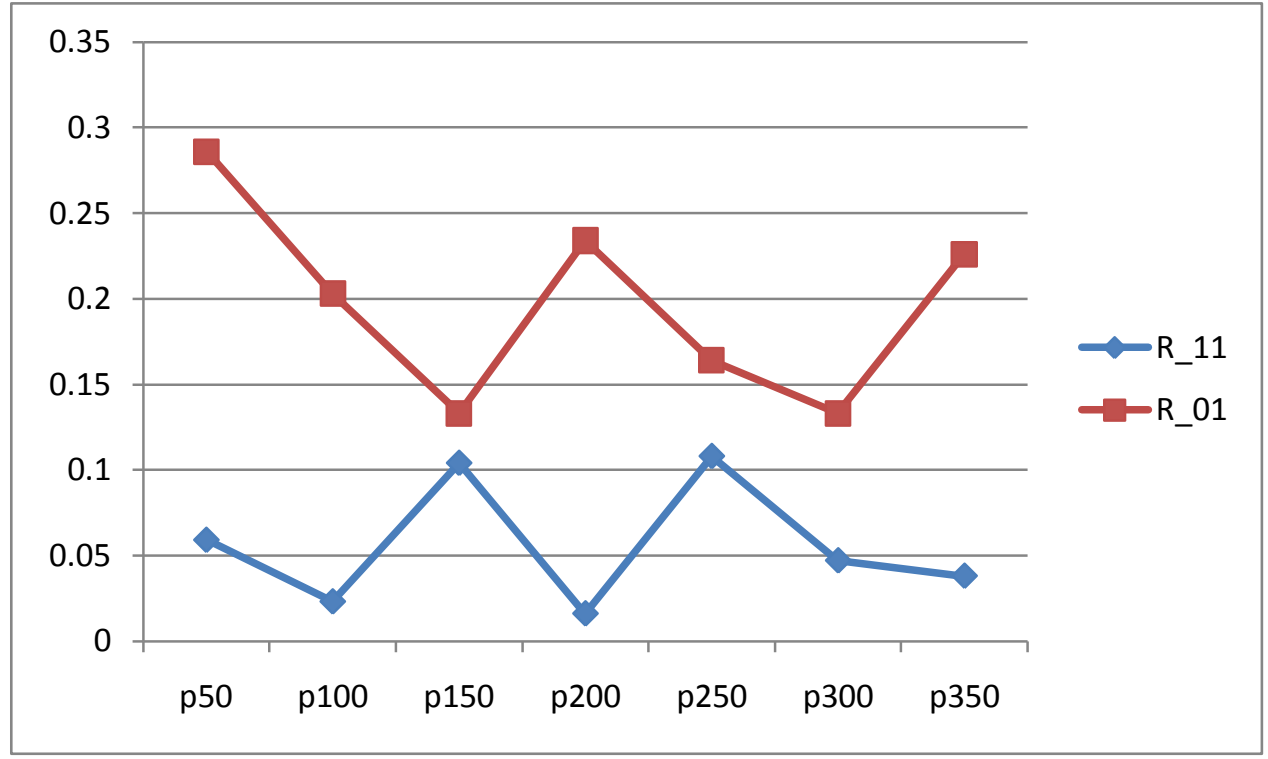

Figure 3-14: Conditional Precipitation of Monongalia across days of year

\section{Monroe}

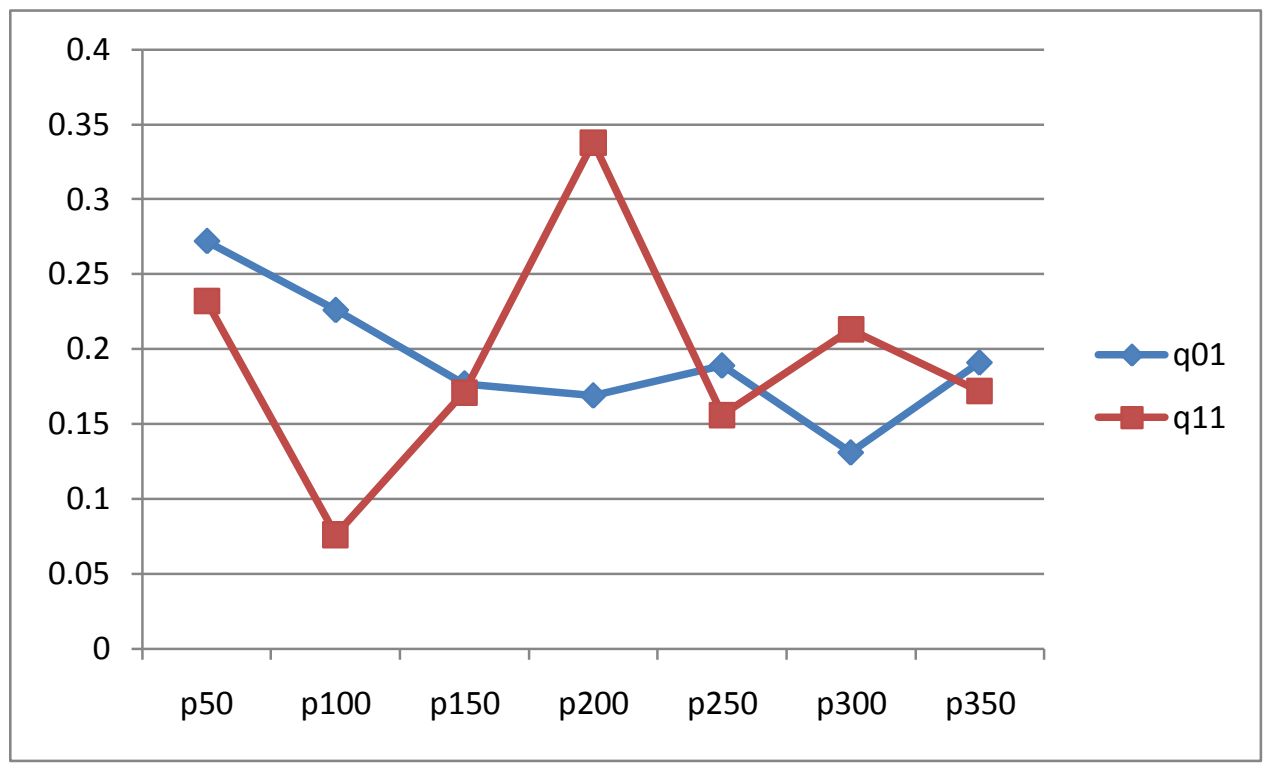

Figure 3-15: transition probability (q01/q11) of Monroe 


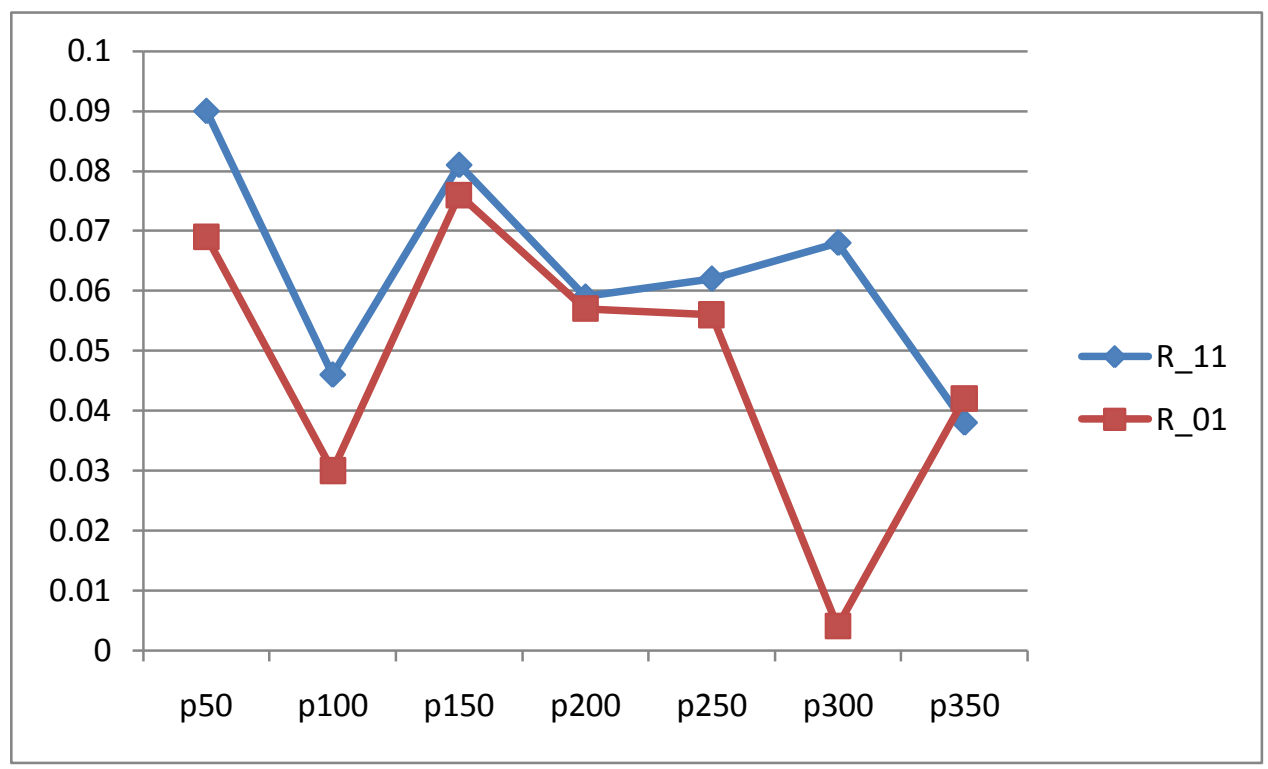

Figure 3-16: conditional precipitation amount of Monroe across days of year

Figures from 3-13 to 3-16 represent the transition probability (i.e. the transition probability from a dry day ( $\mathrm{t}-1)$ to a wet day $(\mathrm{t})$ which is $\mathrm{q}_{01}$ or the transition from a wet day ( $\mathrm{t}$ 1) to a wet day (t) which is $q_{11}$ ) and the conditional precipitation depending on the state of previous day (i.e. whether it was a dry or a wet day) for the weather stations of MonongaliaHartfield and Monroe.. These transition probabilities and conditional precipitation amount have been plotted against the day of the year (e.g. p50 $=50^{\text {th }}$ day, p100 $=100^{\text {th }}$ day and so on).

Tables 3-3. And 3-4. Represent the Gamma and Exponential Distribution Parameter estimates. According to the Kolmogorov-Smirnoff/Anderson-Derling statistics generated by SAS/ETS, Gamma distribution was found to be a better match between gamma and exponential distributions. Further, @Risk had been called to check which distribution best fits the data. It was found that this software validates selection of this distribution choice. 
Table 3-3.: Gamma Distribution Parameter Estimates

\begin{tabular}{|c|c|c|c|c|c|}
\hline Weather Stations & Time Period & Scale parameter, beta or sigma & Shape Parameter, alpha & Mean & Std.Dev \\
\hline Hart Field, Monongalia & August & 1.03 & 3.94 & 4.05 & 2.04 \\
\hline Martinsburg, Berkeley & August & 1.012 & 3.36 & 3.39 & 1.85 \\
\hline Monroe & August & 0.874 & 3.57 & 3.12 & 1.65 \\
\hline Randolph & August & 2.68 & 2.01 & 5.4 & 3.8 \\
\hline
\end{tabular}

Table 3-4. : Exponential Distribution Parameter Estimates

\begin{tabular}{|c|l|l|l|l|}
\hline Weather Station & $\begin{array}{l}\text { Estimated Theta or } \\
\text { Threshold }\end{array}$ & $\begin{array}{l}\text { Sigma or Scale } \\
\text { Parameter }\end{array}$ & Mean & Standard Deviation \\
\hline $\begin{array}{c}\text { Hart Field, } \\
\text { Monongalia }\end{array}$ & 0.908 & 3.14 & 4.04 & 3.14 \\
\hline $\begin{array}{c}\text { Martinsburg, } \\
\text { Berkeley }\end{array}$ & 0.63 & 2.761 & 3.39 & 2.761 \\
\hline Monroe & 0.66 & 2.498 & 3.157 & 2.498 \\
\hline Randolph & 0.948 & 4.398 & 5.346 & 4.398 \\
\hline
\end{tabular}

\subsection{Derivative (Option) Pricing: Classical Methods}

There are four classical methods to price a derivative according to the book "Options, Futures and Other Derivatives" (Hull, 2007). They are briefly described here.

\section{Black-Scholes model}

Fischer Black and Myron Scholes first articulated the Black-Scholes formula in their 1973 paper, "The Pricing of Options and Corporate Liabilities". The fundamental insight of Black-Scholes is that the option is implicitly priced if the stock is traded.

\section{The Black-Scholes Partial Differential Equation (hereinafter B/S PDE):}

Notation:

$S$, the price of the stock

$V(S, t)$, the price of a derivative as a function of time and stock price.

$C(S, t)$ the price of a European call and $P(S, t)$ the price of a European put option. 
$K$, the strike of the option.

$r$, the annualized risk-free interest rate, continuously compounded.

$\mu$, the drift rate of $S$, annualized.

$\sigma$, the volatility of the stock; this is the square root of the quadratic variation of the stock's log price process.

$t$ a time in years; we generally use now $=0$, expiry $=\mathrm{T}$.

$\Pi$, the value of a portfolio.

$R$, the accumulated profit or loss following a delta-hedging trading strategy.

As per the model assumptions above, we assume that the underlying asset (typically the stock) follows a Geometric Brownian motion/Wiener Process. That is,

$\mathrm{dS}=\mu \mathrm{Sdt}+\sigma \mathrm{SdW}$

where $W$ is Brownian - the $d W$ term here stands in for any and all sources of uncertainty in the price history of a stock. The payoff of an option $V(S, T)$ at maturity is known. To find its value at an earlier time we need to know how $V$ evolves as a function of $S$ and $T$. By Itō's lemma for two variables we have:

$d V=\left(\mu S \frac{\partial V}{\partial S}+\frac{\partial V}{\partial t}+\frac{1}{2} \sigma^{2} S^{2} \frac{\partial^{2} V}{\partial S^{2}}\right) d t+\sigma S \frac{\partial V}{\partial S} d W$

After careful manipulation, one finally obtains the B-S PDE which is given by:

$\frac{\partial V}{\partial t}+\frac{1}{2} \sigma^{2} S^{2} \frac{\partial^{2} V}{\partial S^{2}}+r S \frac{\partial V}{\partial S}-r V=0$

This partial differential equation holds whenever $V$ is twice differentiable with respect to $S$ and once with respect to $t$.

\section{Model Assumptions:}


The Black-Scholes model of the market for a particular equity makes the following explicit assumptions:

- It is possible to borrow and lend cash at a known constant risk-free interest rate. This restriction has been removed in later extensions of the model.

- The price follows a Geometric Brownian motion with constant drift and volatility. This often implies the validity of the efficient-market hypothesis.

- There are no transaction costs or taxes.

- Returns from the security follow a log-normal distribution.

- The stock does not pay a dividend. All securities are perfectly divisible (i.e. it is possible to buy any fraction of a share).

- There are no restrictions on short selling ${ }^{3}$.

- There is no arbitrage opportunity

- Options use the European exercise terms, which dictate that options may only be exercised on the day of expiration.

Several of these assumptions of the original model have been removed in subsequent extensions of the model. Modern versions account for changing interest rates (Merton, 1976), transaction costs and taxes (Ingerson, 1976), and dividend payout (Merton, 1973).

$N(x)$ denotes the standard normal cumulative distribution function,

$$
N(x)=\frac{1}{\sqrt{2 \pi}} \int_{-\infty}^{x} e^{-\frac{x^{2}}{2} d z}
$$

\footnotetext{
${ }^{3}$ Short selling is when the seller borrows stocks from a broker and sells them immediately, speculating that price of stock will decline. When the stock price declines, he buys them back at a lower price and returns them to the broker. Hence, makes a profit by drop in stock price.
} 
$N^{\prime}(x)=\frac{e^{-\frac{x^{2}}{2}}}{\sqrt{2 \pi}}$

$N^{\prime}(x)$ denotes the standard normal probability density function.

\section{The Black Scholes Formula}

The Black Scholes formula calculates the price of European put and call options. It can be obtained by solving the Black-Scholes partial differential equation.

The value of a call option in terms of the Black-Scholes parameters is:

$$
\begin{aligned}
& C(S, t)=S N\left(d_{1}\right)-K e^{-r(T-t)} N\left(d_{2}\right) \\
& d_{1}=\frac{\ln \left(\frac{S}{K}\right)+\left(r+\frac{\sigma^{2}}{2}\right)(T-t)}{\sigma \sqrt{T-t}} \\
& d_{2}=d_{1}-\sigma \sqrt{T-t}
\end{aligned}
$$

The price of a put option is:

$$
P(S, t)=K e^{-r(T-t)}-S+\left(S N\left(d_{1}\right)-K e^{-r(T-t)} N\left(d_{2}\right)\right)=K e^{-r(T-t)}-S+C(S, t)
$$

For both, as above:

- $\mathrm{N}(\bullet)$ is the cumulative distribution function of the standard normal distribution

- $\mathrm{T}-\mathrm{t}$ is the time to maturity

- $\quad \mathrm{S}$ is the spot price of the underlying asset

- $\mathrm{K}$ is the strike price

- $\quad \mathrm{r}$ is the risk free rate (annual rate, expressed in terms of continuous compounding)

- $\sigma$ is the volatility in the log-returns of the underlying 


\section{Interpretation}

$N\left(d_{1}\right)$ and $N\left(d_{2}\right)$ are the probabilities of the option expiring in-the-money under the equivalent exponential martingale probability measure (numéraire $=$ stock) and the equivalent martingale probability measure (numéraire $=$ risk free asset), respectively. The equivalent martingale probability measure is also called the risk-neutral probability measure. Note that both of these are probabilities in a measured theoretic sense, and neither of these is the true probability of expiring in-the-money under the real probability measure.

\section{Monte Carlo Methods}

The Monte Carlo option model uses Monte Carlo methods to calculate the value of an option with multiple sources of uncertainty or with complicated features. In general the technique is to generate several thousand possible (but random) price paths for the underlying index via simulation, and then calculate the associated exercise value (i.e. "payoff") of the option for each path. These payoffs are then averaged and discounted to today, and this result is the value of the option today.

\section{Binomial Method}

The binomial options pricing model (BPM) provides a generalized numerical method for the valuation of options. The binomial model was first proposed by Cox, Ross and Rubinstein (1979). Essentially, the model uses a "discrete-time" model of the varying price over time of the underlying financial instrument. The Binomial options pricing model approach is widely used as it is able to handle a variety of conditions for which other models cannot easily be applied. This is largely because the BPM models the underlying instrument over time - as opposed to at a particular point. So this model can be used to value American options which can be exercised at any point and Bermudan options which can be exercised at various points. The model is also relatively simple, mathematically, and can therefore be readily implemented in software like 
MATLAB (or even in spreadsheet). Although slower than the Black-Scholes formula, it is considered more accurate, particularly for longer-dated options, and options on securities with dividend payments. For these reasons, various versions of the binomial model are widely used by practitioners in the options markets.

\section{Finite Difference Techniques}

Finite difference methods for option pricing are a numerical method used in mathematical finance for the valuation of options using finite difference methods. The method was first applied to option pricing by Eduardo Schwartz in 1977. Finite difference methods can solve derivative pricing problems that have, in general, the same level of complexity as those problems solved by tree approaches (BPM), and are therefore usually employed only when other approaches are inappropriate. At the same time, like tree-based methods, this approach is limited in terms of the number of underlying variables, and for problems with multiple dimensions, Monte Carlo methods for option pricing are usually preferred. This approach is based on the fact that the evolution of the option value can be modeled using a partial differential equation (for example Black-Scholes PDE discussed above). Once in this form, a finite difference model can be derived, and the valuation obtained.

\subsection{Weather Derivative Valuation: A Review of Literature and Methods}

Since the inception of the weather derivatives market, the number and type of market participants have continuously expanded. A range of market participants including investment banks, insurance firms, energy companies to hedge funds offer weather risk management products. Each participant has its own distinct product line and pricing models (Sloan, Palmer, and Burrow, 2002). These pricing models very often follow either one of the basic models described in the earlier section but they come with more standardized form. In other words, these 
contracts are commonly valued by the expected payoff under the physical measure, discounted at the risk-free rate, i.e., an actuarial approach (Davis, 2001). This is the fundamental concept behind any asset valuation. Subsequently, various alternative pricing mechanisms, as off-shoots of the fundamentals, have been developed and applied for the incomplete markets, including super-replication (El Karoui and Queens, 1995), quadratic approaches (Follmer and Sondermann, 1986; Schweitzer, 1988, 1991; Bouleau and Lamberton, 1989; Duffie and Richardson, 1991), quantile hedging and shortfall minimization (Cvitanic, 1998; Follmer and Leukert, 1999, 2000), the marginal utility approach (Davis, 1998), and indifference pricing (Hodges and Neuberger, 1989; Davis, Panas, and Zariphopoulou, 1993). The incomplete financial market models that acknowledge both the hedgeable and unhedgeable risks are more appropriate for the valuation of weather derivatives.

Some researchers have also attempted to explore the problem of weather derivative valuation in the incomplete market. In one of the earliest papers in weather derivative valuation, by Cao and Wei (2000), the authors propose and implement an equilibrium valuation framework for weather derivatives. This paper generalizes the Lucas model of 1978 to include weather as a fundamental variable in the economy. The numerical analyses provided in this paper show that the market price of risk associated with the temperature variable is insignificant in most cases, especially when risk aversion is high or when the aggregate dividend process is close to a random walk. Davis (2001) explores weather derivative pricing using the marginal utility approach, based on the assumption that agents in the weather derivatives market are not representative but face very specific weather risks. Brockett, Wang, and Yang (2003) adopt an incomplete market pricing model, namely the indifference valuation approach, to analyze the valuation of weather derivatives. The indifference valuation approach starts from the economic 
principle of certainty equivalent (discussed in essay I), but is modified and extended to accommodate partial hedging in the financial market. In a mean-variance framework (again discussed in essay I), the relationship between weather derivatives indifference prices and the transaction volume has been examined, and a number of conditions are obtained when the actuarial price is not an appropriate valuation for weather derivatives. By comparing the buyers and sellers indifference prices, the authors also examine the conditions for the viability of the weather derivatives market. In addition, by examining explicitly the distributional impacts of all the stochastic variables involved, Brockett, Wang, and Yang investigate the impacts of partial hedging, natural hedges, basis risk, quantity risk, and price risk on the investors indifference prices.

In practice, most issuers of weather contracts write a large portfolio to take advantage of geographic diversification and hedging effects. For hedgers, they would desire to use a combination of standardized weather contracts and basis weather derivatives designed to hedge the basis risk (mostly geographic basis risk) of the standardized contracts. Single contracts can be priced in the context of the marginal changes they cause to the portfolio rather than on their stand-alone performance (Jewson et al., 2002). Also in a mean-variance framework, Brockett et al. (2003) adopt the indifference pricing approach to examine the portfolio effects on weather derivatives indifference prices. In this article, portfolio effects are defined as the effects on the indifference prices of weather derivatives when investors usually hold a portfolio of weather derivatives and other financial assets. Their simulation results illustrate how the magnitude of the portfolio effects is related to the correlation between weather indexes, between weather indexes and the payoff of the risky assets in the financial market, and to some other distributional characteristics of the relevant stochastic variables, as well as to the payoff structures of the 
weather contracts already in the investor's portfolio. This is a growing area in research and a pricing model can be made as complex as one wishes with adequate understanding of basic nature of pay-outs, stochastic calculus and derivatives. In the present essay, a simple pricing exercise has been carried out as discussed in the following section.

Since weather derivatives do not have any underlying index like stock in stock options which trades in a primary market, valuation of a weather derivative is very different from the four approaches discussed above. Monte Carlo approach can always be used to price any type of derivative contract. According to Jewson et al., 2005, there are two different pricing approaches available. They are discussed as follows:

\section{'Burn' Analysis}

Burn analysis or just 'burn' is based, very simply, on the idea of evaluating how a contract would have performed in previous years (Jewson, 2002). It explicitly does not include fitting distributions and using Monte Carlo simulations. Although there are cases when other methods may be more accurate or give more information, burn analysis is a good first step in pricing almost any contract. Burn analysis had been performed in this essay to price Rainfall contracts over month of August using cumulative August precipitation as the underlying index using four weather station data of West Virginia over 1971-2000. So in essence, I had 30 cumulative August rainfall observations. I averaged out the respective call and put prices utilizing the 5 different tick sizes (in $\$$ terms) which essentially give me the Rainfall call \& put premiums as per Burn analysis in $\$$ denomination.

\section{Monte Carlo Simulation}

Since traditional models like Black-Scholes are not applicable to value weather based contracts, the best approach would be to use Monte Carlo simulation. It starts out by simulating the various paths the underlying variable (here the weather variable) would take using a parametric 
assumption and generates a substantial number of observations (1,000s) and then averages them using a discount rate to find the contract value at the expiration date. Both the Burn and Monte Carlo simulation have been utilized in this essay to find the optimal value of a rainfall based call option for West Virginia (as described in section 3.20) using weather data of 4 weather stations in West Virginia.

\subsection{Previous Applications of Precipitation Contracts/Derivatives}

Precipitation, both in the form of rain and snow, exerts a significant impact on the revenue of many businesses. Farming is an obvious example. Drought or flood can both adversely affect the crop yield. Too little rainfall affects the quantity of forage produced and hence affects the grassfed producers. They are forced to sell their cattle earlier in the market or send them to the feedlot. By the same token, outdoor recreational parties (e.g. golf, skiing etc.) have much to worry about excess precipitation or the lack of it. Hydroelectric generators are very keen on the accumulative precipitation over any given time period. Lack of precipitation means a low water level in reservoirs, which in turn means a shortage of power supply. The reduced power output not only leads to a loss of revenue, sometimes it also means purchasing power at unfavorable prices from other generators in order to make up the supply shortage. Some of the deals that have been constructed in precipitation derivatives include the Southern hydro partnership versus Credit Lyonnais Rouse Derivatives (Artemis.bm/index.htm). Southern Hydro Partners (SHP) is a hydroelectric power generator in South East Australia, with most of its facilities located in Victoria and New South Wales. With rainfall levels being significantly below the historical average for several years, the company decided to enter into a precipitation contract with Credit Lyonnais Rouse Derivatives or Calyon in 2003. SHP's primary goal was to stabilize cash flows and revenue. The precipitation contract was structured as a collar (described earlier) whereby 
SHP would receive payments from Calyon should the rainfall be lower than a specified threshold level and pay Calyon should the rainfall be above a certain level.

Another precipitation deal was struck between Sacramento Municipal Utility District (SMUD) versus Aquila (Cao, 2004). SMUD is America's sixth largest community-owned electric utility in terms of customers served. The utility generates half of its electricity and buys the rest. The generated portion is primarily from hydroelectric and cogeneration power plants. In September 2000, SMUD entered into a five-year precipitation contract with Aquila to protect against low rainfall levels. The contract was structured as a collar (described earlier) whereby Aquila would pay SMUD annually up to $\$ 20$ million when the water flow through the hydro plants is below a certain amount while SMUD would pay Aquila $\$ 20$ million in years when precipitation is abundant. To further reduce the cost of hedging, the payments to Aquila were capped at $\$ 50$ million.

Another example is a precipitation contract between golf course operators versus Societie Generale (sgcib.com/). A golf course owner entered into a derivative contract with Societie Generale, SG, after assessing that his business suffers when the weather was wet. The structured deal covered the period from May to September, within which the golf course owner would receive compensation should the total number of rainy days go beyond 50. Specifically, once the number of days with rainfall of more than a millimeter passed 50, SG would pay for every wet day.

Similarly, World Bank is also proposing to introduce index based weather derivative contracts in developing countries to reduce the impact of drought (artemis.bm/blog/2008/06/25/world-bank-to-offer-index-based-weather-derivative-contracts/). World Bank proposes to offer financial intermediation services to low-income client countries of 
the International Development Association (IDA) and will add to the range of risk-management tools available to middle income client countries of the International Bank for Reconstruction and Development (IBRD). Malawi is expected to be the first country to take advantage of this new offer. The World Bank is expected to work as the intermediatary by entering into mirroring transactions with the client country and a financial market counterpart. In the event of a severe weather event, client countries, such as Malawi, would receive a payout from the Bank, the total value of which would be based on an index used as an estimate of the financial impact. This would be funded with the payout that the World Bank would receive from the mirroring transaction.

\subsection{A Pricing Exercise by 'Burn' and Monte Carlo Simulation for the Current Essay}

Assuming a parametric distribution (both gamma and exponential), 10,000 observations have been iterated and pricing of the contract has been calculated for different strike/exercise rates of $1.5,2.0,2.5,3.0,3.5$ and 4.0 inches of rainfall with a tick size or liability of $\$ 1000$ per contract. Table 3-4 shows the sample pricing/valuation at expiration for a call option and Table 3-5shows the sample pricing/valuation at expiration for a put option utilizing four weather stations data from West Virginia for both burn and Monte Carlo exercises.

\section{CALL PRICING}

\section{Terms of this contract}

- Period: August, Measure: Cumulative Monthly Rainfall

- Exercise/Strike Levels: 1.50 inches, 2.00 inches, 2.50 inches, 3.00 inches, 3.50 inches and 4.00 inches.

- $\quad$ Tick/Contract Size: \$1000/inch, Limit: \$3000.

- Interest Rate: 5\% Annual Compounding. 
- Locations: Martinsburg, Monongalia, Monroe, Randolph of West Virginia.

Table 3-5.: Call Price in $\$$ (Strike/Exercise rate is in rain inches)

\begin{tabular}{|c|c|c|c|c|c|c|c|}
\hline Valuation Type & $\begin{array}{l}\text { Exercise/Strike } \\
\text { Rate }\end{array}$ & 1.50 & 2.00 & 2.50 & 3.00 & 3.50 & 4.00 \\
\hline \multirow[t]{4}{*}{ Burn } & Martinsburg & 1953.67 & 1542.00 & 1197.67 & 935.55 & 727.67 & 567.67 \\
\hline & Monongalia & 2564.84 & 2101.16 & 1712.26 & 1359.68 & 1069.35 & 832.9 \\
\hline & Monroe & 1689.31 & 1307.24 & 987.24 & 711.03 & 490.69 & 338.62 \\
\hline & Randolph & 2778.96 & 2310.00 & 1887.24 & 1498.28 & 1128.62 & 853.10 \\
\hline \multirow{4}{*}{$\begin{array}{l}\text { Monte Carlo } \\
\text { (Gamma } \\
\text { Distribution) }\end{array}$} & Martinsburg & 1926.93 & 1521.41 & 1176.19 & 892.69 & 665.93 & 488.83 \\
\hline & Monongalia & 2490.16 & 2044.48 & 1644.46 & 1298.74 & 1008.79 & 771.25 \\
\hline & Monroe & 2127.71 & 1705.50 & 1338.81 & 1031.72 & 781.73 & 583.15 \\
\hline & Randolph & 2368.45 & 2109.67 & 1544.32 & 1324.78 & 897.65 & 533.25 \\
\hline \multirow{5}{*}{$\begin{array}{l}\text { Monte Carlo } \\
\text { (Exponential } \\
\text { Distribution) }\end{array}$} & Martinsburg & & & & & & \\
\hline & & 1621.32 & 1355.95 & 1135.97 & 951.76 & 798.20 & 669.01 \\
\hline & Monongalia & 1971.46 & 1686.23 & 1443.12 & 1235.22 & 1058.41 & 907.68 \\
\hline & Monroe & 1347.34 & 1100.42 & 897.88 & 731.57 & 595.35 & 484.99 \\
\hline & Randolph & 3066.82 & 2732.02 & 2432.71 & 2166.91 & 1929.82 & 1718.32 \\
\hline
\end{tabular}

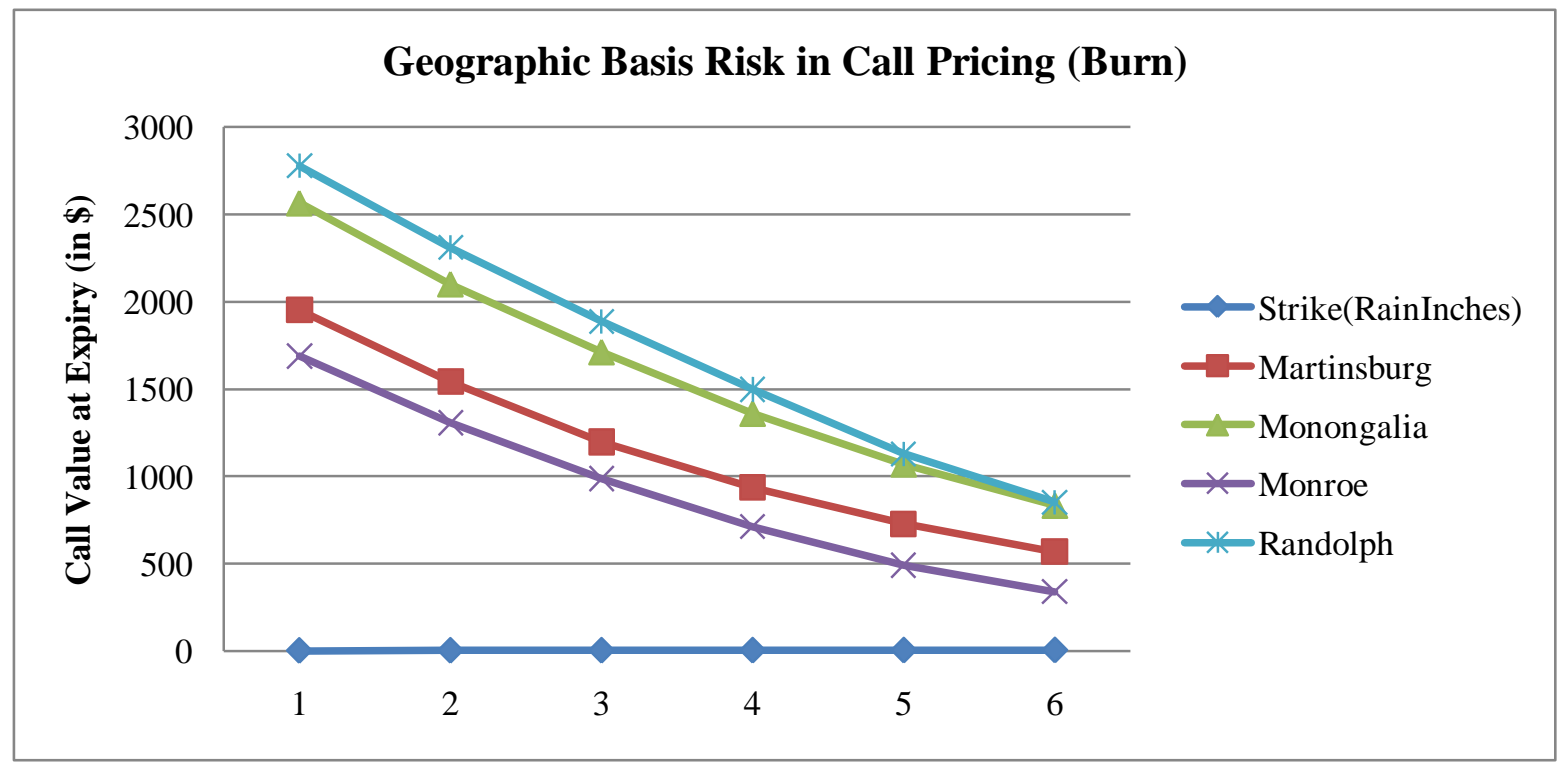

Figure 3-17.: Geographic Basis Risk in Call Value (Burn Pricing) 


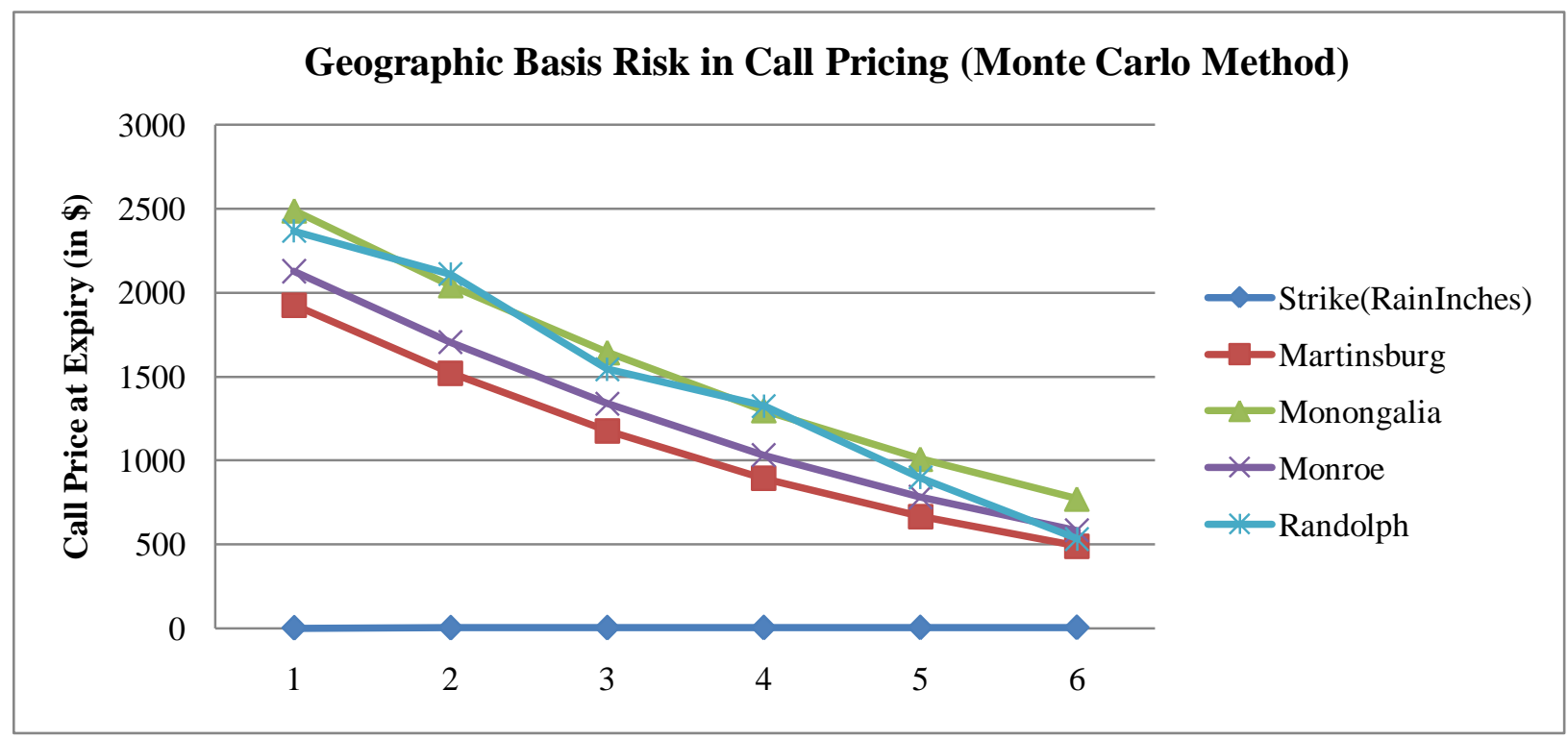

Figure 3-18: Geographic Basis Risk in Call Value (Monte Carlo Pricing)

\section{PUT PRICING}

\section{Terms of this Contract}

- Period: August, Measure: Cumulative Monthly Rainfall

- Exercise/Strike Levels: 6.00 inches, 6.50 inches, 7.00 inches, 7.50 inches and 8.00 inches.

- Tick/Contract Size: \$1000/inch. Limit: No Limit.

- Interest Rate: 5\% Annual Compounding

- Locations: Martinsburg, Monongalia, Monroe, Randolph of West Virginia. 
Table 3-6.: Put Value at Expiry in \$ (Strike/Exercise rate is in precipitation inches)

\begin{tabular}{|l|l|l|l|l|l|l|l|}
\hline $\begin{array}{l}\text { Valuation } \\
\text { Type }\end{array}$ & $\begin{array}{l}\text { Exercise/Strike } \\
\text { Rate (in Inches of } \\
\text { Precipitation) }\end{array}$ & 6.00 & 6.50 & 7.00 & 7.50 & 8.00 & 8.50 \\
\hline Burn & Martinsburg & 2677.333 & 3136.00 & 3606.333 & 4101.333 & 4601.333 & 5101.333 \\
\hline & Monongalia & 2292.90 & 2728.38 & 3163.87 & 3599.67 & 4051.29 & 4518.71 \\
\hline & Monroe & 2892.414 & 3378.621 & 3878.621 & 4378.621 & 4878.621 & 5378.621 \\
\hline & Randolph & 1958.33 & 2375.00 & 2797.00 & 3240.00 & 3690.66 & 4157.33 \\
\hline $\begin{array}{l}\text { Monte Carlo } \\
\text { (Gamma) }\end{array}$ & Martinsburg & 2728.78 & 3189.19 & 3657.65 & 4141.18 & 4626.44 & 5116.437 \\
\hline & Monongalia & 2189.37 & 2623.21 & 3071.43 & 3533.50 & 4006.07 & 4484.665 \\
\hline & Monroe & 2921.96 & 3425.25 & 3907.13 & 4395.70 & 4886.90 & 5381.848 \\
\hline & Randolph & 1838.66 & 2176.10 & 2533.52 & 2907.56 & 3297.37 & 3701.027 \\
\hline $\begin{array}{l}\text { Monte Carlo } \\
\text { (Exponential) }\end{array}$ & Martinsburg & 3536.74 & 3983.45 & 4437.36 & 4897.69 & 5364.34 & 5838.00 \\
\hline & Monongalia & 3314.12 & 3744.13 & 4182.96 & 4630.40 & 5084.65 & 5546.85 \\
\hline & Monroe & 4170.08 & 3712.40 & 4633.97 & 5103.83 & 5579.71 & 6058.80 \\
\hline & Randolph & 2719.76 & 3096.43 & 3486.60 & 3888.54 & 4301.38 & 4722.87 \\
\hline
\end{tabular}

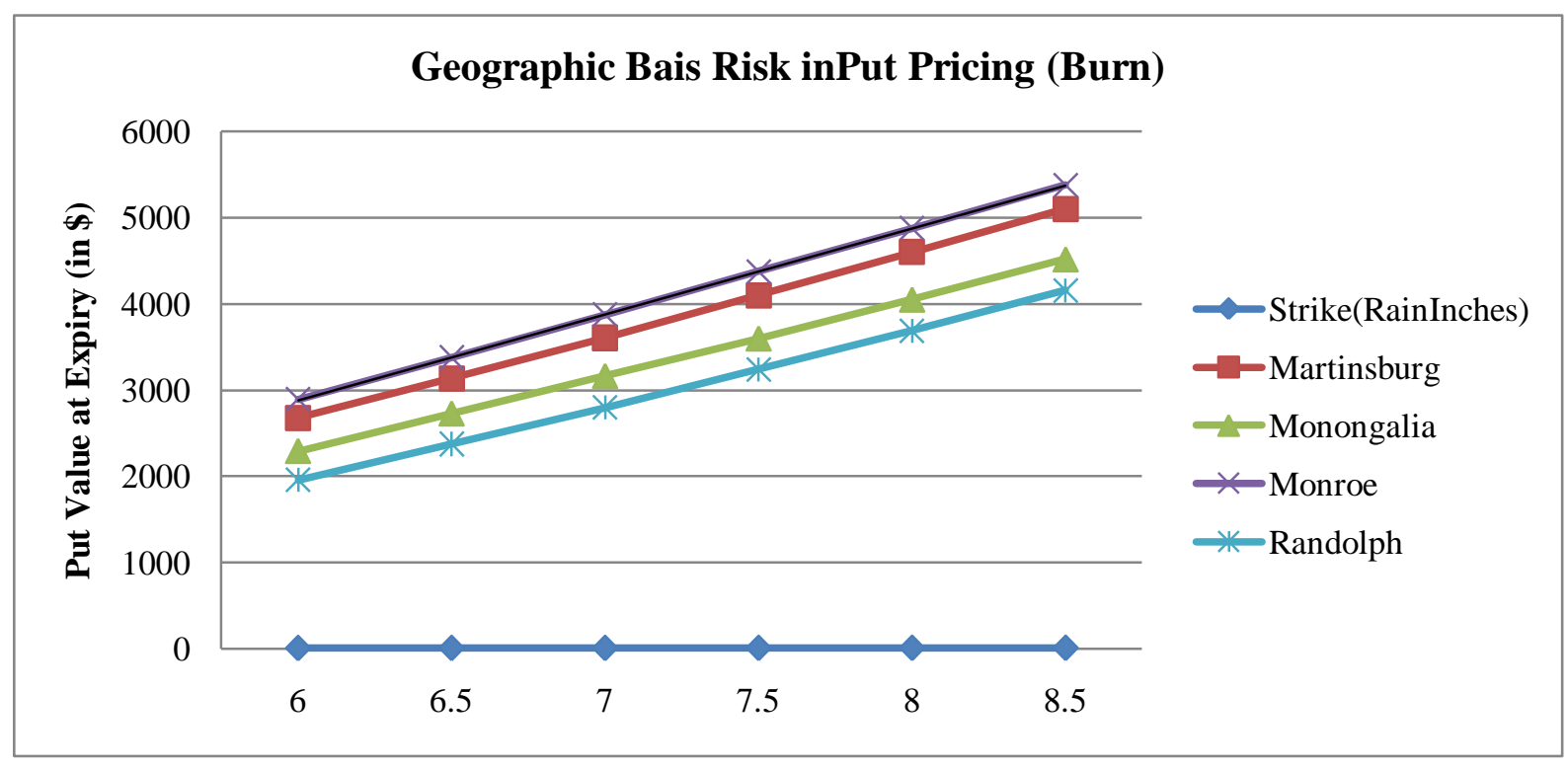

Figure 3-19.: Geographic Basis Risk in Put Pricing (Burn Pricing). 


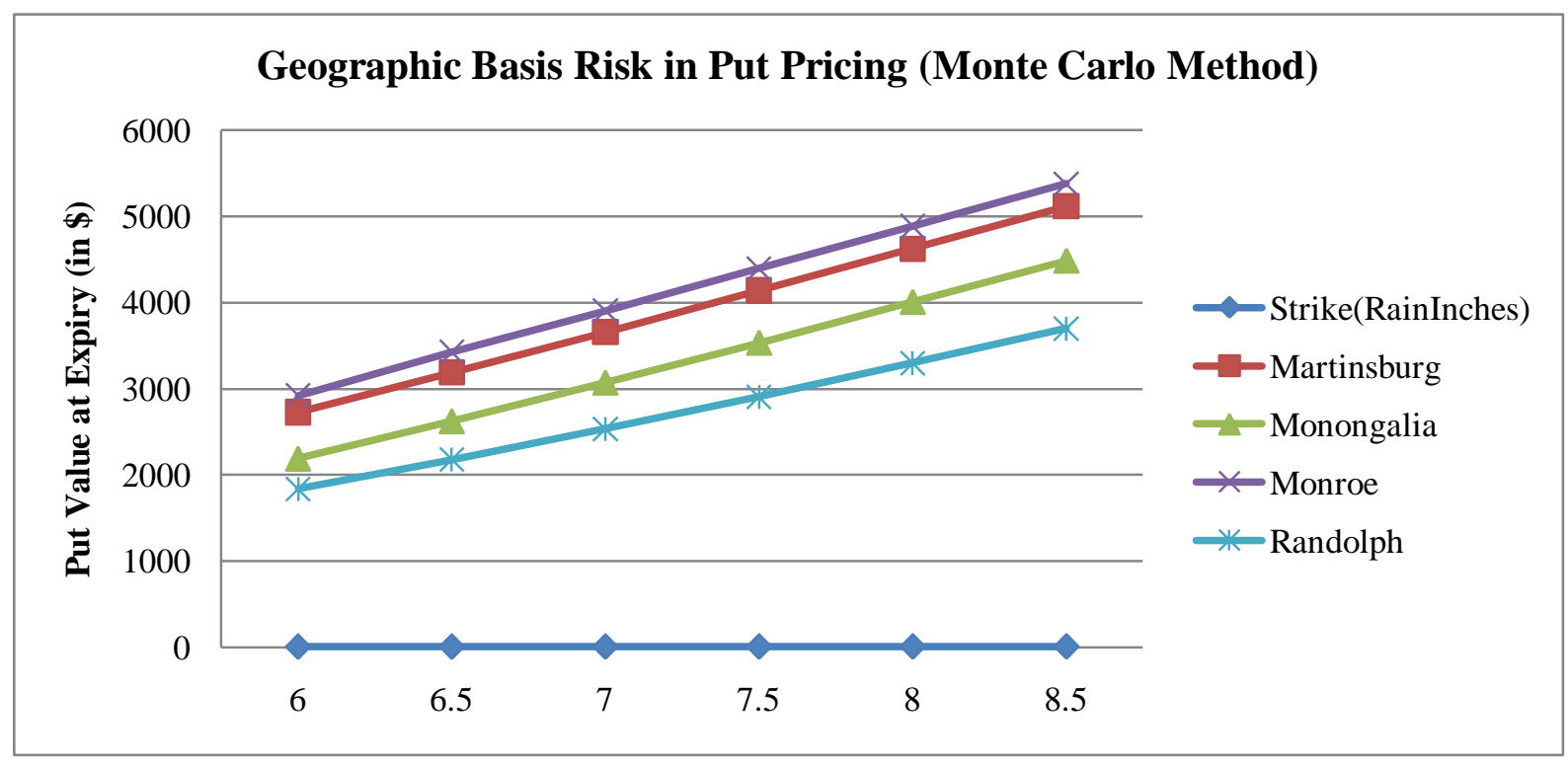

Figure 3-20.: Geographic Basis Risk in Put Pricing (Burn Pricing). 


\subsection{Option Calculator}

Subsequently, an option calculator has been developed in Visual Basic (VB.Net). The logical flow chart is as follows:

User chooses the given choice of option type (call/put), pricing type (Burn or Monte Carlo) and the reference weather station (Monongalia/Martinsburg/Monroe/Randolph) from the pull down menu.

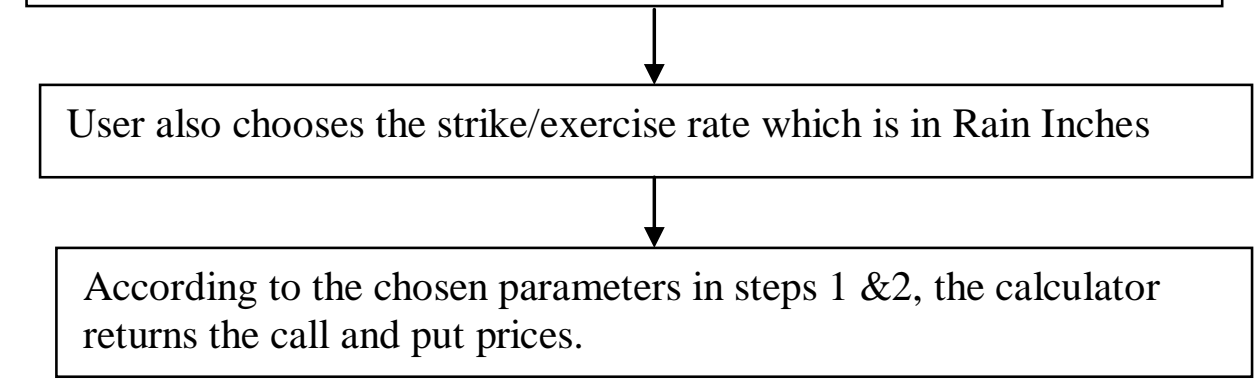

Figure 3-21.: Flowchart of Option Calculator

\subsection{Possible Applications of the Structured Contract}

In the previous section I have discussed historical precipitation/rainfall based deals. Following that, the structured precipitation contract for West Virginia has potential application for crop insurance, event insurance, golf course operators etc. Outdoor recreational operators may find it useful to hedge the bottom line of their business. Another possible application is event insurance, which is becoming popular of late. In this case, basis risk between the contract and the economic exposure needs to be matched as accurately as possible.

\subsection{Limitation of the Study and Suggested Derivative Strategies for Grass Fed Producers}

The main limitation of the current study is lack of a hedging effectiveness of the structured rainfall based contract. It was not possible to determine how much of the revenue of a grass fed producer is actually affected by too much or too little rainfall. In our email based survey 7 out of 16 respondents mentioned that drought or lack of rainfall affects their production related 
activities. The next step in this study should be a hedging analysis using the structured contract and revenue generated by the grass fed producer. Though it was not possible to complete such study during the time frame of this study, the following steps should constitute the basic framework.

\begin{tabular}{l}
$\begin{array}{l}\text { Calculate Grass fed Revenue of a standard farm in a normal } \\
\text { year, drought year and year with excess rainfall. }\end{array}$ \\
$\begin{array}{l}\text { Generate several scenarios of each of such years by } \\
\text { simulation. }\end{array}$ \\
$\begin{array}{l}\text { For each normal/drought or excess rainfall year, calculate } \\
\text { revenue as generated either by selling or buying } \\
\text { structured contract for each rain inches. }\end{array}$ \\
\hline \\
\hline Recommend the best strategy to these producers. \\
\hline
\end{tabular}

Figure 3-22.: Framework for Hedging effectiveness with Rainfall Option for Grass fed producers

\section{Derivative Strategy:}

Either a put/collar or strangle could work for these producers. A put option is identical to insurance. A collar works well as it means buying a put and selling a call, so it could be possible that no initial investment is necessary. Appendix E contains the pay-off diagrams of these strategies.

\subsection{Conclusion}

As the overall market for weather derivatives grows, contracts on precipitation are gradually making their way into the scene. However, compared with temperature derivatives, the market share of precipitation derivatives is still very minimal. This slow growth does not indicate a lack of interest or demand. There is enough demand from the farmers, hydro-power generators, sports event managers or recreation parks, the end users of this derivative product. However, there is lack of interest from the financial services firms or investment banks to provide a quote or 
underwrite the risk. This disinterest comes from difficulty of rainfall modeling. Though various models of rainfall have been described in earlier sections, it is still a challenge to model extreme rainfall like a flood or severe drought and incorporate the spatial variability that generally comes with rainfall or any other weather phenomena. Application of a Kalman filter could be a possible solution towards better rainfall modeling. Non parametric methods can be investigated and modern techniques like artificial intelligence or support vector machines can be used for simulation/forecasting purposes. Extreme value theory (EVT) can be investigated to model extreme rainfall.

Albeit being new in the derivative world, interest in weather derivatives is certainly growing. Increase in the volume of trade at CME is a proof of this interest. However, the spatial nature of weather phenomena and the associated geographic basis risk are big roadblocks towards a major acceptance of this unique weather related risk management tool by companies hit by adverse weather. This rainfall/precipitation based contract, when made available either as OTC or exchange (CME) traded, could provide yet another risk management strategy to manage businesses suffering from too-little (use of put-option) or too-much rainfall (use of call option). This could successfully be applied to the grass-fed producers as well. 


\section{LIST OF REFERENCES}

\section{A. Journal Articles}

Black, F. and Scholes, M. 1973. The pricing of Options and Corporate Liabilities. Journal of Political Economy 81:637-654.

Brennan, M.J., and Schwartz, E.S., 1978. Finite Difference Methods and Jump Processes Arising in the Pricing of Contingent Claims: A Synthesis. Journal of Financial and Quantitative Analysis 13 (I-3)3:461-474.

Brockett, P.M., M. Wang, and C. Yang. 2005. Weather Derivatives and Weather Risk Management. Risk Management and Insurance Review 8(1): 127-140.

Brockett, P.M., M. Wang, C. Yang. And H.Zou.2006. Portfolio Effects and Valuation of Weather Derivatives. Financial Review 41: 55-76.

Brody, D.C., J.Syroke, and M.Zervos. 2002. Dynamic Pricing of Weather Derivatives. Quantitative Finance 2: 189-198.

Buchen, P. 2002. Options, Derivatives and Exotics. In 2002 Course Notes - School of Mathematics and Statistics, University of Sydney.

Cao, M., and J.Wei. 2004. Weather Derivatives Valuation and Market Price of Weather Risk. Journal of Futures Markets 24(I-11):1065-1089.

Cao, M., Li, A. and Wei, J. 2004. Precipitation Modeling and Contract Valuation: A Frontier in Weather Derivatives. The Journal of Alternative Investments 7(I-2): 93-99.

Cvitanic, J.1998. Minimizing Expected Loss of Hedging in Incomplete and Constrained Markets, Preprint, Columbia University.

Coe, R. and R.D. Stern. 1982. Fitting models to daily rainfall. Journal of Applied Meteorology 21:1024-1031.

Cox, J.C, S.A. Ross, and M. Rubinstein. 1979. Option Pricing: A Simplified Approach. Journal of Financial Economics 7: 229-264.

Dornier, F., and M. Querfuel.2000. Weather Derivatives Pricing: Caution to the Wind. Energy \& Power Risk Management (Aug.):30-32.

Davis, M. 1998. Option Pricing in Incomplete Markets, Mathematics of Derivative Securities, M. A. H. Dempster and S. R. Pliska, eds. (Cambridge: Cambridge University Press).

Davis, M. 2001. Pricing Weather Derivatives by Marginal Value. Quantitative Finance 1: 
Davis, M., V. Panas, and T. Zariphoupoulou. 1993. European Option Pricing With Transaction Costs. SIAM Journal on Control and Optimization 31: 470-493.

Dubrovsky, M., J. Buchtele, and Z.Zalud. 2004. High-Frequency and Low-Frequency Variability in Stochastic Daily Weather Generator and Its Effect on Agricultural and Hydologic Modeling. Climate Change 63 (I-1, 2):145-179.

Duffie, D., and H. Richardson. 1991. Mean-Variance Hedging in Continuous Time. Annals of Applied Probability 1: 1-15.

El Karoui, N., and M. Queens. 1995. Dynamic Programming and Pricing of Contingent Claims in an Incomplete Market. SIAM Journal on Control and Optimization 33: 29-66.

Follmer, H., and P. Leukert. 1999. Quantile Hedging. Finance and Stochastics 3: 251-73.

Follmer, H., and P. Leukert. 2000. Efficient Hedging: Cost versus Shortfall Risk. Finance and Stochastics 4: 117-46.

Hodges, S., and A. Neuberger. 1989. Optimal Replication of Contingent Claims under Transaction Costs. Review of Futures Markets 8: 222-239.

Jewson, S., A. Brix, and C. Ziehmann. 2002. Getting the Price Right. Global Reinsurance February: 10-15.

Katz, R.W. 1977. Precipitation as a Chain-Dependent Process. Journal of Applied Meteorology16:671-676.

MacDonald, J., J. Perry, M. Ahearn, D. Banker, W. Chambers, C. Dmimtri, N. Key, K. Nelson and L. Southard. Contracts, Markets and Prices. 2004. In USDA, Economic Research Service, Ag Economic Report No. 837, November 2004.

Mahul, O. 2002. Hedging in Futures and Options Markets with Basis Risk. Journal of Futures Markets 22: 59-72.

Martin,S.E., B.J. Bernett and K.H. Coble. 2001. Developing and Pricing Precipitation Insurance. Journal of Agricultural and Resource Economics 26(1):261-274.

McCarl, B.A., and D.A. Bessler. 1989. Estimating an Upper Bound on the Pratt Risk Aversion Coefficient When the Utility Function is Unknown. Australian Journal of Agricultural and Resource Economics 33(I-1):55-63.

Merton, R.C. 1973. Theory of Rational Option Pricing. Bell Journal of Economics and Management 4: 141-183. 
Moreno, M. 2000.Rainfall derivatives. Derivatives Week 10(I-11):6-7.

Ross, Stephen A. 1976. The Arbitrage Theory of Capital Asset Pricing. Journal of Economic Theory 13 (I-3): 341-360.

Schweizer, M. 1991. Option Hedging for Semi martingales. Stochastic Processes and Their Applications, 37: 339-63.

Sharpe, William F. 1964. Capital Asset Prices: A Theory of Market Equilibrium under Conditions of Risk. Journal of Finance 19 (I-3): 425-442.

Sloan, D., L. Palmer, and H. Burrow. 2002. A Broker's View. Global Reinsurance. February: 22-25.

Wilks, D.S. 1989. Conditioning Stochastic Daily Precipitation Models on Total Monthly Precipitation. Water Resources Research 23: 1429-1439.

\section{B. Books/Working Papers}

Campbell, S., and E.X. Diebold, 2003. Weather Forecasting for Weather Derivatives. NBER Working Paper No. W10141.

Cochrane, John, 2000. Asset Pricing. Princeton University Press.

Hull, John, 2008. Options, Futures and Other Derivatives. Sixth Edition. Prentice Hall of India Pvt. Ltd., New Delhi, India.

Benth, F. E., and J. Benth, 2005.The volatility of temperature and pricing of weather derivatives. Dept. of Mathematics, University of Oslo, E-print series, ISSN 0806-2439, no 12/2005.

Jewson, Stephen and A.Brix, 2005. Weather Derivative Valuation: The Meteorological, Statistical, Financial and Mathematical Foundations. Cambridge University Press, Cambridge, UK.

Cambell, S.D and Diebold, F.X,. 2002. Weather Forecasting for Weather Derivatives. Wharton, The Rodney White Centre for Financial Research, University of Pennsylvania.

Dillon, J.L., Anderson, J.R. 1990. The analysis of response in crop and livestock production, Third Edition. Pergamon Press, Armidale, Australia.

Dunis C., L. and Karalis V. 2003. Weather Derivatives Pricing and Filling Analysis for Missing Temperature Data, Liverpool Business School and CIBEF.

Greene, W.H., Econometric Analysis, Fifth Edition, Pearson Education Ltd., New Delhi, India. 
Paul Wilmott. Derivatives: The Theory and Practice of Financial Engineering, First edition, John Wiley \& Sons Ltd., West Sussex, UK .

\section{Websites}

Weather Risk Management Association (WRMA) web site;

Available at: http://www.wrma.org.

Climetrix - An initiative of RMS. Pages: Market News, Market Overview, Weather Data;

Available at: http://www.climetrix.com.

CME Group Website available at: www.cmegroup.com.

Speedwell Weather Systems - Speedwell Derivatives;

Available at: http://www.weatherderivs.com/.

Glossary of Investment related terms/definitions; Available at: www.investopedia.com/

Glossary of Option related definitions; Available at: www.theoptions.net

CME Blog on Weather derivatives; Available at:www.artemis.bm/blog/2008/06/25/world-bankto-offer-index-based-weather-derivative-contracts/

Website of Societie Generale Corporate \& Investment Bank, available at: www.sgcib.com. 


\section{APPENDIX A}

\section{USDA/RMA Coverage Reports used in Essay I}

Date: $06 / 16$

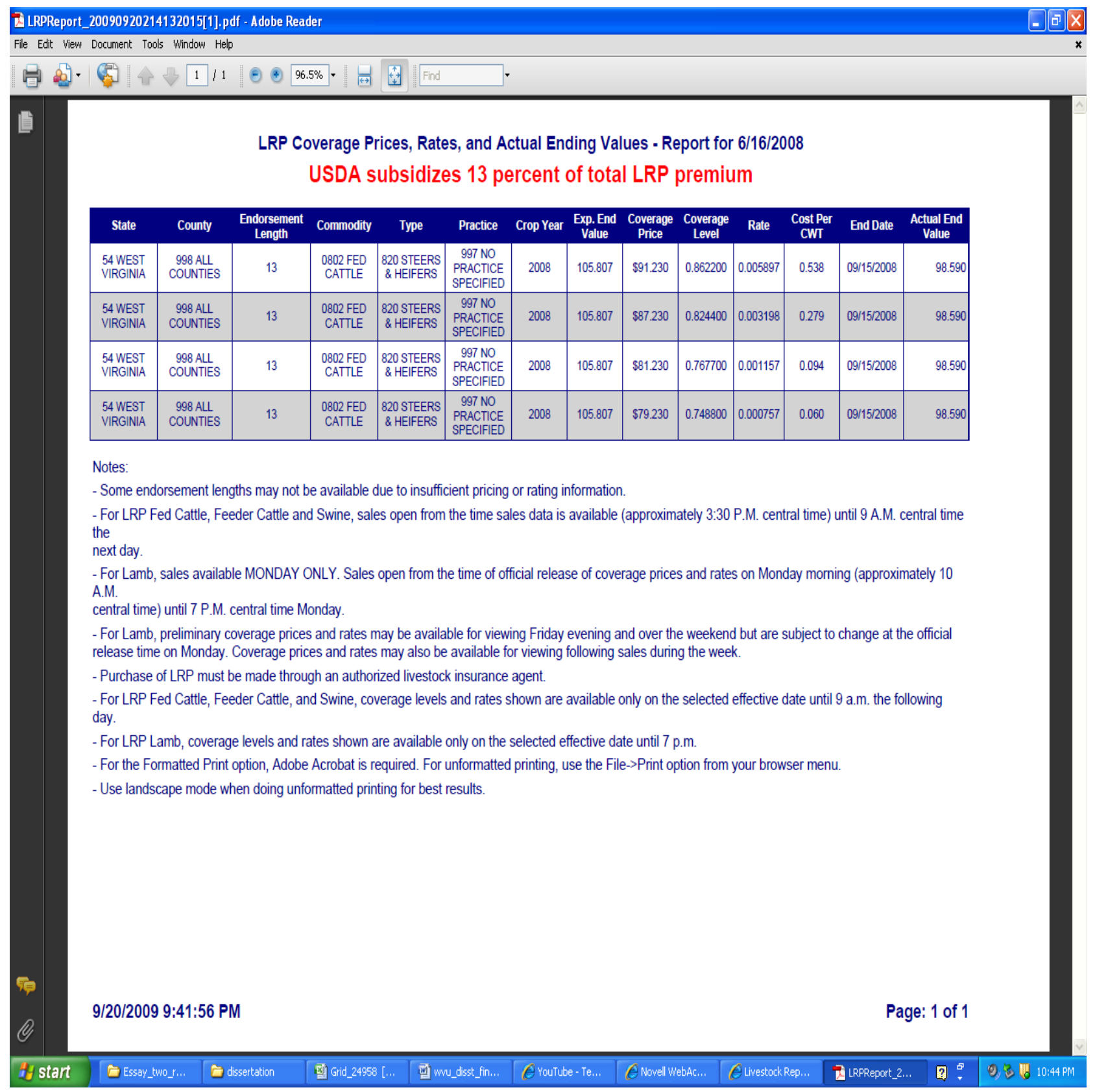

Website: http://www3.rma.usda.gov/apps/livestock_reports/main.aspx 
USDA/RMA Coverage Reports used in Essay I

Date: 05/19

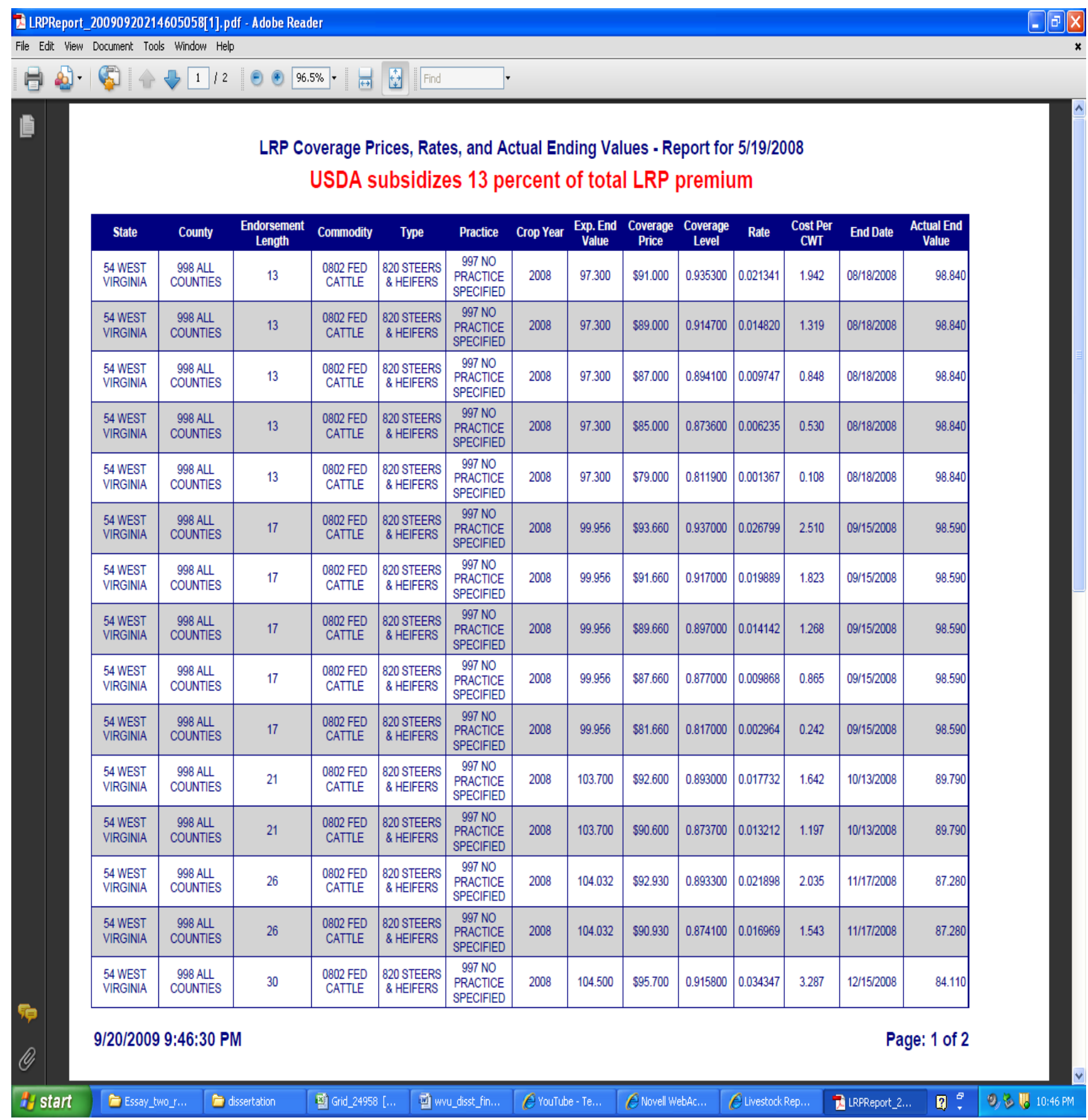

Website: http://www3.rma.usda.gov/apps/livestock_reports/main.aspx 
USDA/RMA Coverage Reports used in Essay I

Date: $04 / 21$

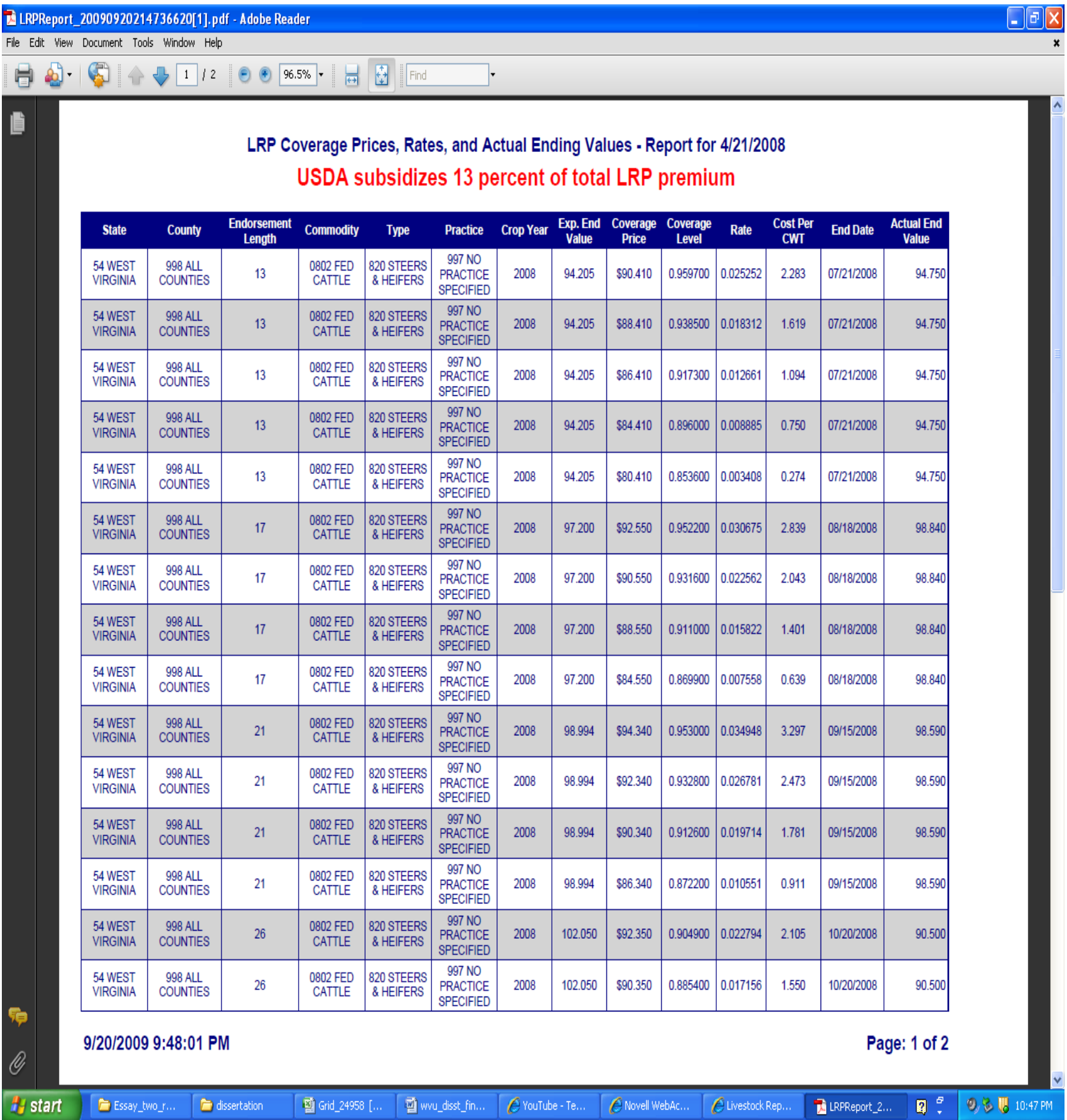

Website: http://www3.rma.usda.gov/apps/livestock_reports/main.aspx 
USDA/RMA Coverage Reports used in Essay I

Date: $03 / 17$

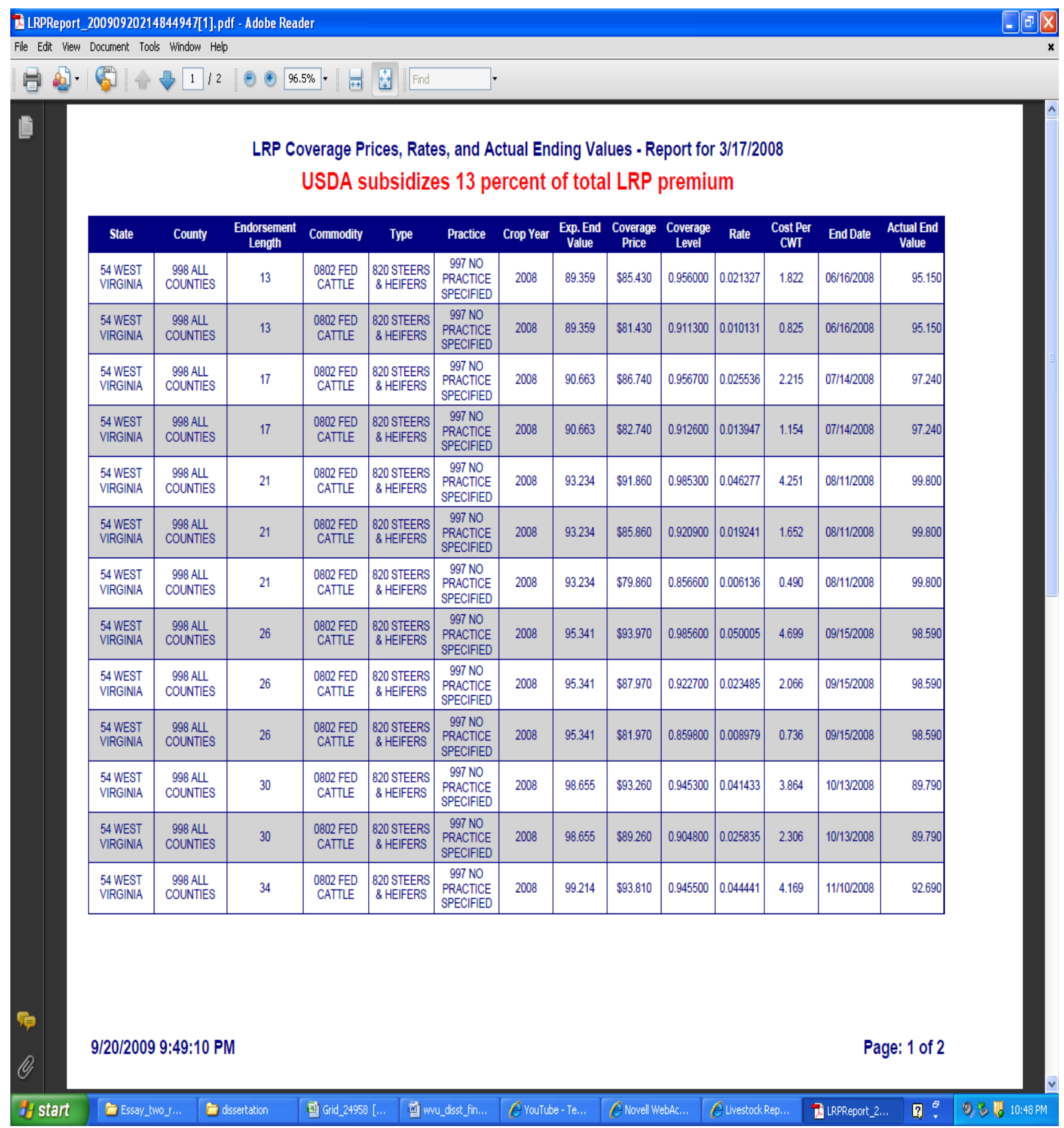

Website: http://www3.rma.usda.gov/apps/livestock_reports/main.aspx 


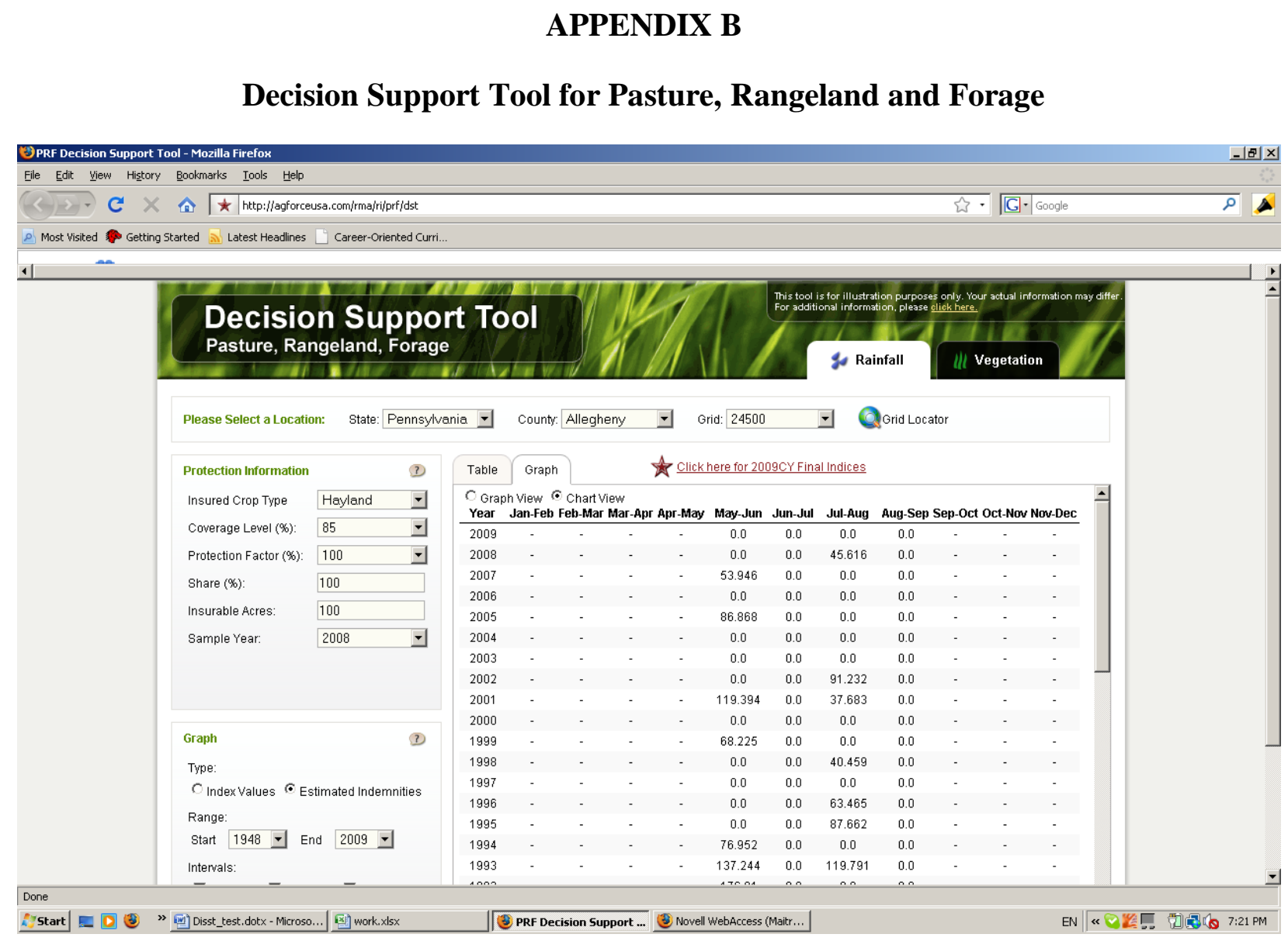

Website: http://www.rma.usda.gov/policies/pasturerangeforage 


\section{APPENDIX C}

Basis Risk in Local Cattle Market vs. AMS 5 Area Market

\begin{tabular}{|c|c|c|c|c|c|c|c|}
\hline Year & $\begin{array}{l}\text { AMS } 5 \\
\text { Market (\$) }\end{array}$ & $\mathrm{VA}(\$)$ & $\operatorname{PA}(\$)$ & Year & $\begin{array}{l}\text { AMS 5 } \\
\text { Market(\$) }\end{array}$ & $\mathrm{VA}(\$)$ & $\mathrm{PA}(\$)$ \\
\hline Apr-05 & 91.91 & 93 & 80.7 & Sep-07 & 92.9 & 88.6 & 78.3 \\
\hline May-05 & 88.76 & 90.2 & 81.6 & Oct-07 & 93.63 & 84.7 & 76.2 \\
\hline Jun-05 & 83.76 & 88.7 & 76.3 & Nov-07 & 92.85 & 76.9 & 72.6 \\
\hline Jul-05 & 80.23 & 84.6 & 72 & Dec-07 & 92.92 & 67.5 & 74.6 \\
\hline Aug-05 & 79.13 & 88.1 & 71.2 & Jan-08 & 90.35 & 65.8 & 74.7 \\
\hline Sep-05 & 86.39 & 88.1 & 72.2 & Feb-08 & 91.15 & 73.9 & 77.1 \\
\hline Oct-05 & 88.87 & 88 & 73.4 & Mar-08 & 89.93 & 79.1 & 77.4 \\
\hline Nov-05 & 90.07 & 84.2 & 73.9 & Apr-08 & 89.96 & 78.5 & 75.9 \\
\hline Dec-05 & 94.45 & 74.9 & 77.6 & May-08 & 94.09 & 79 & 79.1 \\
\hline Jan-06 & 93.42 & 74.8 & 79.1 & Jun-08 & 93.22 & 80.1 & 80.6 \\
\hline Feb-06 & 88.87 & 77 & 77.7 & Jul-08 & 97.24 & 80 & 83.7 \\
\hline Mar-06 & 85.96 & 75.8 & 75.6 & Aug-08 & 99.8 & 82.4 & 83.5 \\
\hline Apr-06 & 82.19 & 77.5 & 73.1 & Sep-08 & 98.59 & 81.7 & 82.6 \\
\hline May-06 & 79.21 & 74.4 & 72.3 & Oct-08 & 89.79 & 74.6 & 76.9 \\
\hline Jun-06 & 80.11 & 77.7 & 73.2 & Nov-08 & 92.69 & 69.8 & 73.9 \\
\hline Jul-06 & 81.39 & 81.3 & 73.1 & Dec-08 & 84.11 & 59.9 & 72.1 \\
\hline Aug-06 & 85.6 & 82.1 & 72.7 & Jan-09 & 83.75 & 65.1 & 73.1 \\
\hline Sep-06 & 87.92 & 86.8 & 74.2 & Feb-09 & 82.76 & 67.2 & 71.4 \\
\hline Oct-06 & 86.89 & 82.4 & 72.5 & Mar-09 & 82.96 & 72.1 & 70.2 \\
\hline Nov-06 & 86.59 & 71.8 & 72.2 & Apr-09 & 88.01 & 76.7 & 72.4 \\
\hline Dec-06 & 85.12 & 62.2 & 72 & May-09 & 85 & 76.1 & 74.9 \\
\hline Jan-07 & 86.77 & 62.7 & 73.9 & Jun-09 & 81.84 & 73 & 71.9 \\
\hline Feb-07 & 90.48 & 68.4 & 76.6 & Jul-09 & 83.88 & 75.7 & 72 \\
\hline Mar-07 & 98.14 & 77.8 & 79.4 & Aug-09 & 81.95 & 74.3 & 71.2 \\
\hline Apr-07 & 96.03 & 84.7 & 81.2 & Sep-09 & 84.23 & 75.4 & 69.7 \\
\hline Мay-07 & 97.59 & 77.8 & 83.6 & Oct-09 & 82.99 & 71.2 & 68.9 \\
\hline Jun-07 & 89.79 & 75 & 79 & Nov-09 & 84.03 & 67.1 & 69.3 \\
\hline Jul-07 & 89.71 & 79.7 & 77.3 & Dec-09 & 81.2 & 62.7 & 69.4 \\
\hline Aug-07 & 90.3 & 83.4 & 78.5 & & & & \\
\hline
\end{tabular}

Data Source: NASS/Market News, VA/PA. 


\section{APPENDIX D}

Actual LRP end value (\$) from $15^{\text {th }}$ Sep. $-15^{\text {th }}$ Oct., 2005 to $15^{\text {th }}$ Sep.-15 ${ }^{\text {th }}$ Oct., 2009

\begin{tabular}{|r|r|r|r|r|l|}
\hline Date & 2005 & 2006 & 2007 & 2008 & 2009 \\
\hline 15-Sep & 86.39 & 88.7 & 92.9 & 98.59 & 84.23 \\
\hline 16-Sep & 86.39 & 88.7 & 92.9 & 98.59 & 84.23 \\
\hline 17-Sep & 86.39 & 88.7 & 92.9 & 98.59 & 84.23 \\
\hline 18-Sep & 86.39 & 88.75 & 93.99 & 98.59 & 84.23 \\
\hline 19-Sep & 86.21 & 88.75 & 93.99 & 98.59 & 84.23 \\
\hline 20-Sep & 86.21 & 88.75 & 93.99 & 98.59 & 84.23 \\
\hline 21-Sep & 86.21 & 88.75 & 93.99 & 98.59 & 84.31 \\
\hline 22-Sep & 86.21 & 88.75 & 93.99 & 98.47 & 84.31 \\
\hline 23-Sep & 86.21 & 88.75 & 93.99 & 98.47 & 84.31 \\
\hline 24-Sep & 86.21 & 88.75 & 95.77 & 98.47 & 84.31 \\
\hline 25-Sep & 86.21 & 91.19 & 95.77 & 98.47 & 84.31 \\
\hline 26-Sep & 86.98 & 91.19 & 95.77 & 98.47 & N/A \\
\hline 27-Sep & 86.98 & 91.19 & 95.77 & N/A & N/A \\
\hline 28-Sep & 86.98 & 91.19 & 95.77 & N/A & N/A \\
\hline 29-Sep & 86.98 & 91.19 & 91.75 & 96.32 & N/A \\
\hline 30-Sep & 86.98 & 91.19 & 91.75 & 96.32 & N/A \\
\hline 1-Oct & 86.98 & 91.19 & 91.75 & 96.32 & N/A \\
\hline 2-Oct & 86.98 & 90.22 & 91.75 & 96.32 & N/A \\
\hline 3-Oct & 86.98 & 90.22 & 91.75 & 91.79 & N/A \\
\hline 4-Oct & 88.86 & 90.22 & 91.75 & 91.79 & N/A \\
\hline 5-Oct & 88.86 & 90.22 & 91.75 & 91.79 & N/A \\
\hline 6-Oct & 88.86 & 90.22 & 92.21 & 91.79 & N/A \\
\hline 7-Oct & 88.86 & 90.22 & 92.21 & 91.79 & 81.35 \\
\hline 8-Oct & 88.86 & 90.22 & 92.21 & 91.79 & 81.35 \\
\hline 9-Oct & 88.86 & 87.92 & 92.21 & 91.79 & 81.35 \\
\hline 10-Oct & 88.86 & 87.92 & 92.21 & 91.79 & N/A \\
\hline 11-Oct & 88.86 & 87.92 & 92.21 & N/A & N/A \\
\hline 12-Oct & 88.87 & 87.92 & 92.21 & N/A & 82.99 \\
\hline 13-Oct & 88.87 & 87.83 & 92.21 & N/A & 82.99 \\
\hline 14-Oct & 88.87 & 87.83 & 92.21 & 89.79 & 82.99 \\
\hline 15-Oct & 87.68 & 87.83 & 92.21 & 89.79 & 82.99 \\
\hline
\end{tabular}

Website: http://www3.rma.usda.gov/apps/livestock_reports/main.aspx 


\section{APPENDIX E}

\section{List of Weather Stations and Weather Futures Contracts}

(Data Source: CME website, www.cmegroup.com)

\begin{tabular}{|c|c|c|}
\hline Weather Location & Station & WBAN \\
\hline Atlanta Hartsfield Airport & KATL & 13874 \\
\hline $\begin{array}{c}\text { Baltimore-Washington International } \\
\text { Airport }\end{array}$ & KBWL & 93721 \\
\hline Boston Logan Airport & KBOS & 14739 \\
\hline Chicago O'Hare Intl Airport & KORD & 94846 \\
\hline Cincinnati Northern Kentucky Airport & KCVG & 93814 \\
\hline Dallas Fort Worth Airport & KDFW & 3927 \\
\hline Des Moines Intl Airport & KDSM & 94847 \\
\hline Detroit Metro Airport & KDTW & 14933 \\
\hline Houston Bush Intercontinental Airport & KIAH & 12960 \\
\hline Kansas City International Airport & KMCI & 3947 \\
\hline Las Vegas McCarran Airport & KLAS & 23169 \\
\hline $\begin{array}{c}\text { Minneapolis-St.Paul International } \\
\text { Airport }\end{array}$ & KMSP & 14922 \\
\hline New York La Guardia Airport & KLGA & 14732 \\
\hline Philadelphia International Airport & KPHL & 13739 \\
\hline Portland International Airport & KPDX & 24229 \\
\hline Sacramento Executive Airport & KSAC & 23232 \\
\hline Salt Lake City International Airport & KSLC & 24127 \\
\hline Tucson International Airport & KTUS & 23160 \\
\hline
\end{tabular}




\section{APPENDIX F}

\section{CME Weather Contract Specifications}

\begin{tabular}{|c|c|}
\hline Trading Hours & $\begin{array}{l}\text { Futures products trade electronically only on CME Globex from } \\
\text { Monday through Friday from } 3: 45 \text { PM to } 3: 15 \text { PM } \\
\text { (central time) of the following day and on Sundays } \\
\text { from 5:30 PM to 3:15 PM. On the last trading day } \\
\text { they trade until } 9 \text { AM. Option products trade only } \\
\text { Monday through Friday from } 8: 15 \text { AM to } 3: 15 \text { PM on } \\
\text { the CME trading floor. }\end{array}$ \\
\hline Contract Size & $\begin{array}{l}\$ 100 \text { times the monthly index. The monthly index is provided by } \\
\text { the Earth Satellite Corporation. }\end{array}$ \\
\hline Minimum Tick Fluctuation & One degree day index point \\
\hline Settlement & $\begin{array}{l}\text { Cash settled. All contracts that remain open at the termination of } \\
\text { trading of a particular contract shall be settled using } \\
\text { the respective CME Degree Days Index for that city } \\
\text { and that contract season, using the methodology in } \\
\text { effect on that date, on the first Exchange business day } \\
\text { that is at least two calendar days after the derivatives } \\
\text { contract month }\end{array}$ \\
\hline Maximum Order Size & $\begin{array}{l}10,000 \text { contracts net long or short in all contract months } \\
\text { combined. }\end{array}$ \\
\hline Trading Venue & $\begin{array}{l}\text { Only options can be traded via open outcry; the futures products } \\
\text { are traded exclusively on the CME Globex electronic } \\
\text { trading platform. }\end{array}$ \\
\hline
\end{tabular}

(Data Source: CME website, www.cmegroup.com) 


\section{APPENDIX G}

\section{Glossary of few option types}

\begin{tabular}{|c|c|}
\hline Type & Description \\
\hline Vanilla & A standard option like European or American option. \\
\hline European & $\begin{array}{l}\text { An option which can only be exercised at the end of its term i.e. at the expiry } \\
\text { date. }\end{array}$ \\
\hline American & An option which can only be exercised at any date in its term. \\
\hline Asian & $\begin{array}{l}\text { This is like vanilla option only the strike price is the arithmetic average of the } \\
\text { underlying during its lifetime. }\end{array}$ \\
\hline Bermudan & $\begin{array}{l}\text { An option that can be exercised only during a part of its lifetime, a similar } \\
\text { example is the employee stock option, since employees will exercise this option } \\
\text { only during their tenure with the firm. }\end{array}$ \\
\hline $\begin{array}{l}\text { Binary or } \\
\text { Digital }\end{array}$ & $\begin{array}{l}\text { Options with binary or discontinuous pay-off. Cash or nothing call or put option } \\
\text { pays-off a certain amount of money or nothing if the underlying is above or } \\
\text { below a fixed level. Asset or nothing options delivers the underlying if the asset } \\
\text { is above or below a fixed level or otherwise have zero pay-off. }\end{array}$ \\
\hline
\end{tabular}




\section{APPENDIX H}

\section{Pay-off Diagram for Combinations}

A. CAP (or Call/Put with a maximum pay-off)

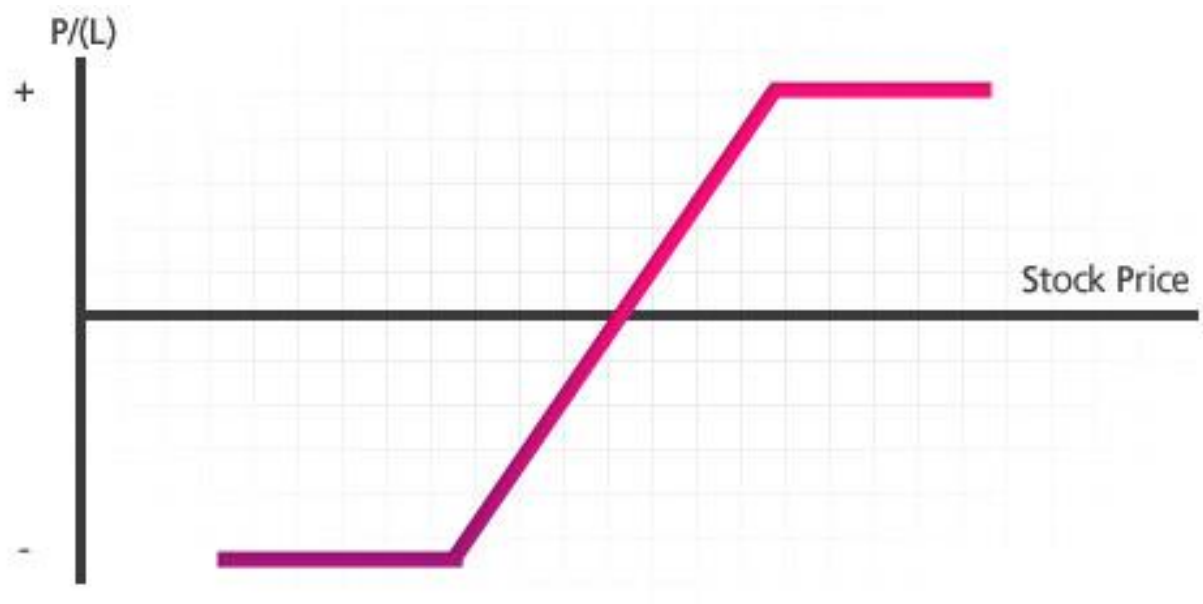

Figure: Long Call with a maximum pay-off. (Source: www.theoptions.net)

B. Straddle

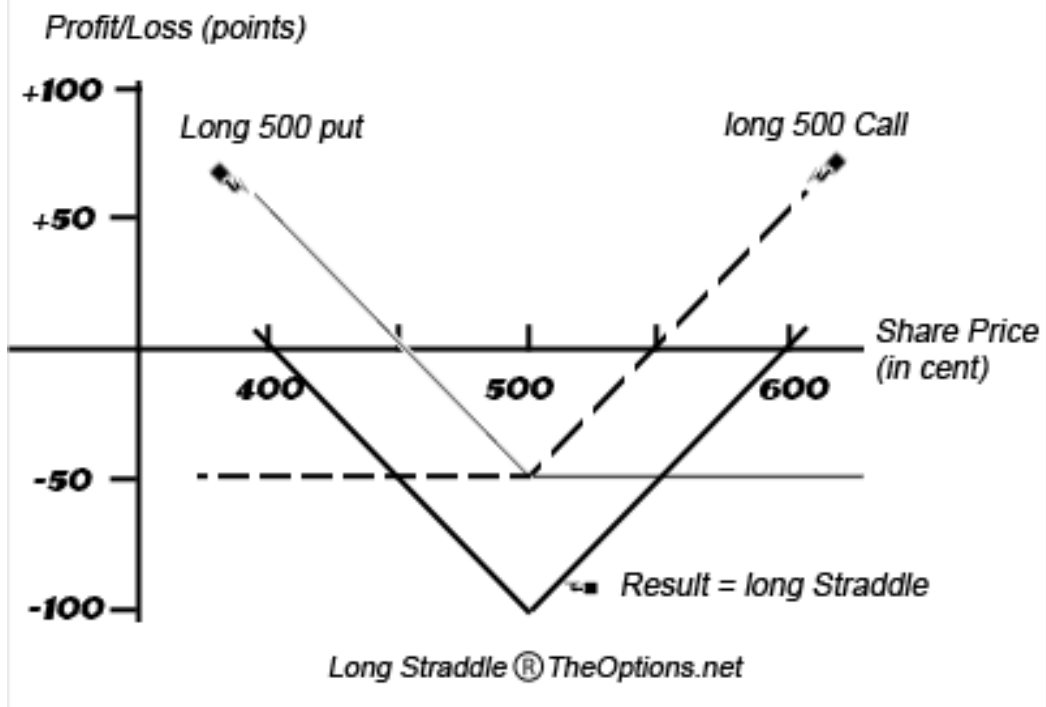

Figure: Straddle. (Source: www.theoptions.net)

Here, strike rate for long call and long put was 500 points. 
C. Strangle

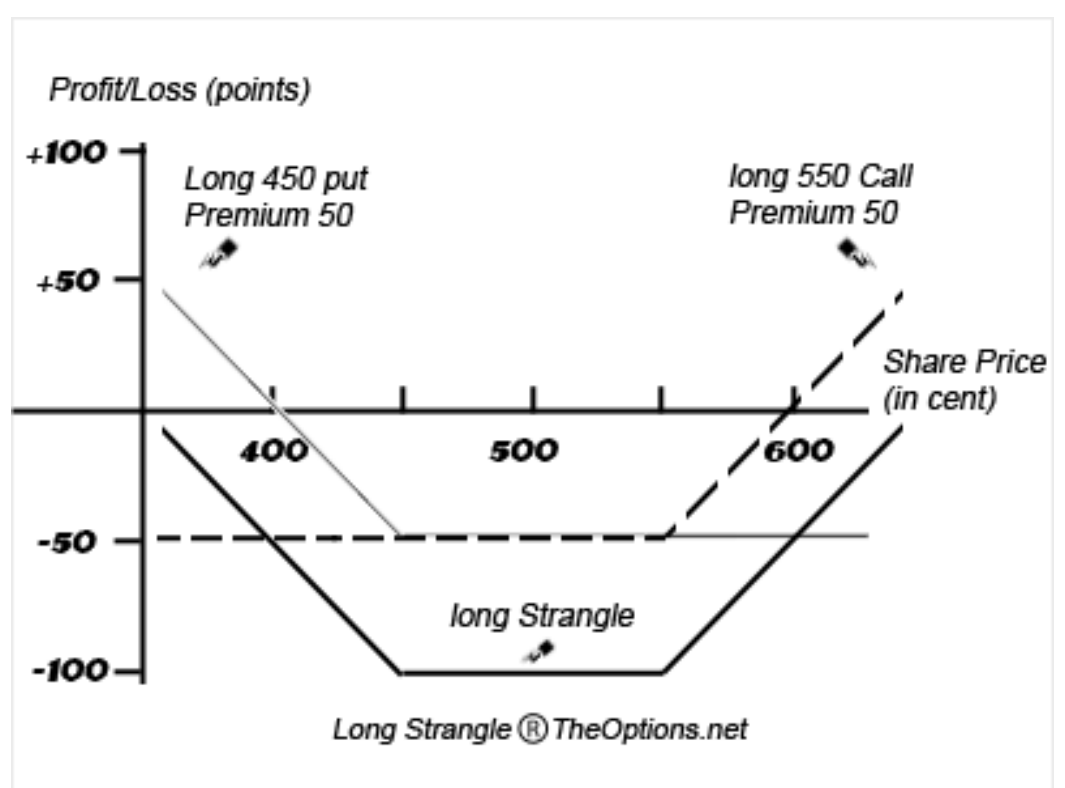

Figure: Strangle.

(Source: www.theoptions.net)

Here, strike rate for long call was 500 points and strike rate for long put was 400 points.

D. Collar

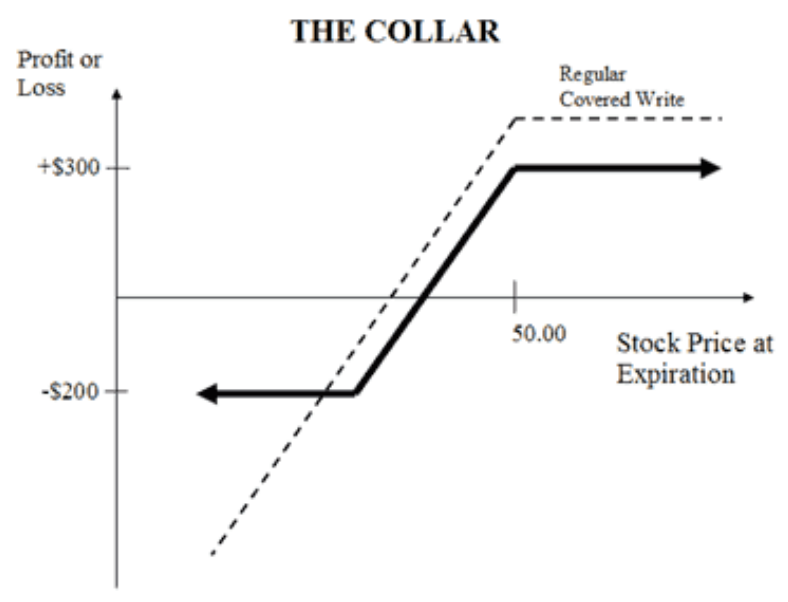

Figure: Collar. (Source: www.theoptions.net). 


\section{APPENDIX I}

\section{RAINFALL OPTION CALCULATOR}

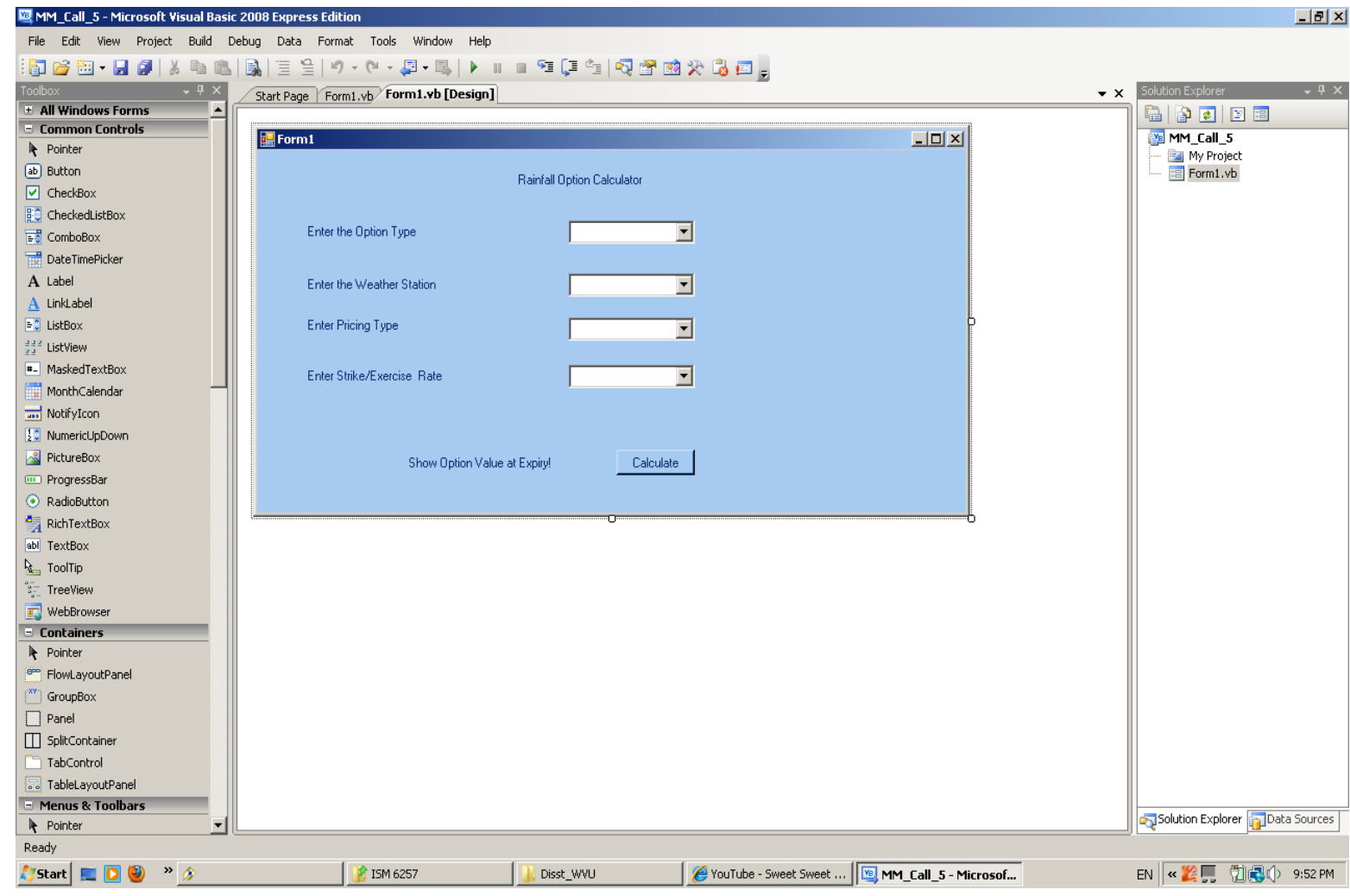




\section{APPENDIX K \\ Grass-Fed Survey Questionnaire}

1. How large is your average annual calf crop and in any given year, what portion of your calf crop do you retain for grass-finishing?

2. What types of grass-fed beef products do you sell (whole carcasses, half carcasses, quarter carcasses, individual cuts, and ground beef)? What portion of your sales volume is made up of each of these product types?

3. If you sell individual cuts or ground beef, are they sold frozen?

4. What is your estimated monthly sales volume (in pounds) for at least the past five years?

5. Do your prices reflect retail-level beef prices or are they fixed from year to year?

6. Do you think climatic/weather variability has been a factor that affects your farm's production performance? 